\title{
Accretion Processes in Astrophysics (A General Review)
}

\section{Franco Giovannelli*†}

INAF - Istituto di Astrofisica e Planetologia Spaziali, Via del Fosso del Cavaliere, 100, 00133

Roma, Italy

E-mail: Eranco.giovannellidiaps.inaf.it

I will update the review published in the proceedings of the first Accretion Processes in Cosmic Sources (APCS) 2016 workshop (Giovannelli \& Sabau-Graziati, 2016). A part of the figures used are the same since there are no reasons to justify a drastic change in the structure of the present review.

However, it is my intention to show that every component of the Universe is bound to every other component and that the comfortable classifications that are usually used are nothing more than artifices to demonstrate their apparent diversity.

In reality, we move from "one class of cosmic sources" to another continuously.

In this review I will discuss the following arguments:

- Antecedent Fact

- Introduction

- Accretion in Young Stellar Objects

- Accretion in Planets

- Accretion in White Dwarfs

- Accretion in Neutron Stars and Black Holes

- Accretion onto the Oceans

- Accretion in the Space

- Conclusions

Accretion Processes in Cosmic Sources - II - APCS2018

3-8 September 2018

Saint Petersburg, Russian Federation

* Speaker.

${ }^{\dagger}$ A footnote may follow. 


\section{Antecedent fact}

In this era - very troubled by major events, such as the overpopulation of the planet, the unstoppable migratory flows, due to the looting of the poorest countries, the run-up to nuclear weapons in the false illusion that peace can be guaranteed by weapons, poisoning apparently unstoppable of the planet - humanity is going along a wrong path.

So where are we going and for what purpose?

All human efforts since long time are inspired by the attempt to answer these fundamental questions:

- Where do we come from?

- Where are we going?

- Why we are here?

Indeed, Lev Nikolayevich Tolstoy in his novel "Anna Karenina" describes the conversation between Anna and Levin, in which she says: Who are we? Where do we come from? Where do we go?

The same questions were posed by Paul Gauguin in 1897 when he painted his famous picture, preserved in the Museum of Fine Arts in Boston and shown in Figure 1. The figures present humanity in the different states of life, from infancy to old age, in a mysterious and indeterminate nature.

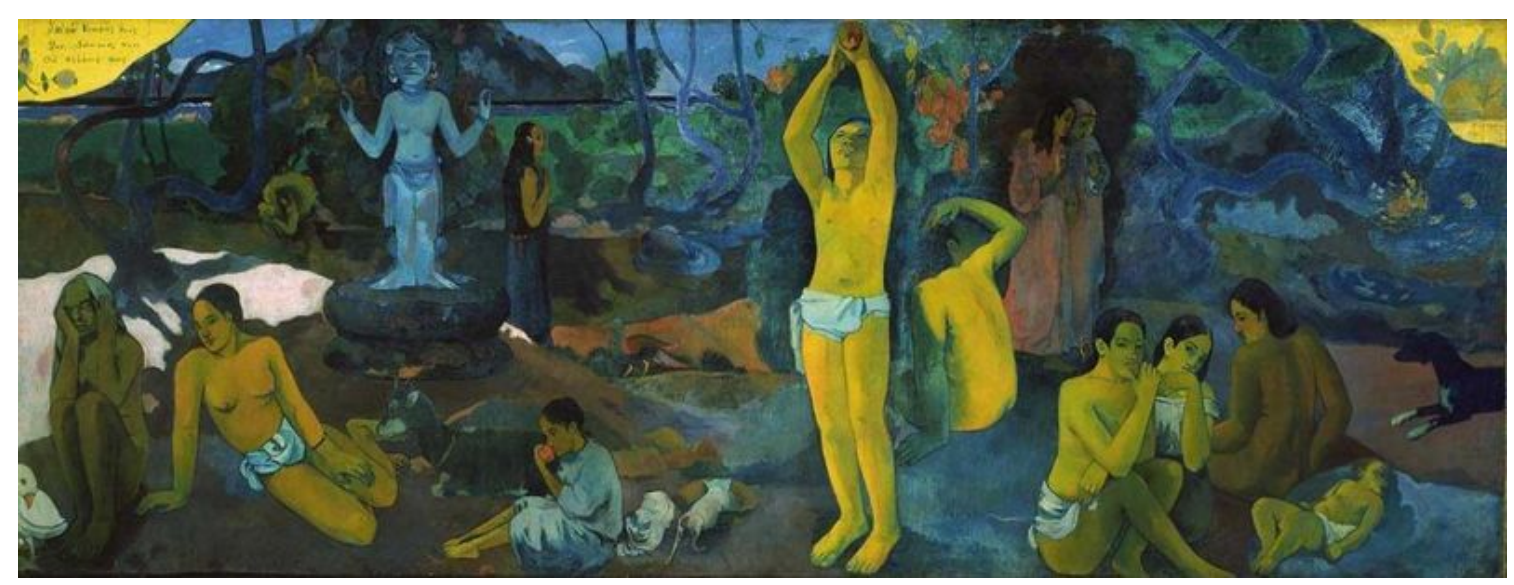

Figure 1: Where do we come from? Who we are? Where do we go? (Paul Gauguin, 1897, Museum of Fine Arts, Boston, Ma, USA).

Indeed, the canvas has a horizontal development with a reading path that goes right to left. Along this direction, Gauguin has a series of figures that essentially repeats the "Allegories of the Ages of Life". From the newborn in the right corner we reach the dark woman on the left passing through the various seasons of life. The woman at the center, who almost divides the picture into two, symbolizes the moment of life in which the fruits are gathered, an obvious allegory of the moment of procreation. The old woman on the far left, already present in other compositions by 
Gauguin, in her fetal position with her hands next to her face, does not really symbolize only old age but above all the fear of death.

But extraordinary in this context is above all the setting. The path of life takes place in a garden that really knows of Eden. As if to say that, according to Gauguin, after all, life and reality are not bad, were it not for the anguish of not knowing for sure what it is all about.

With this picture the sense of uneasiness and instability, typical of the artist and man Gauguin, appears at the end as an endless journey, because it is aimed at goals that are not of this world. And so his escape from the West to the paradises of the South Seas, after all, is nothing but the metaphor, not figured but real, of the perennial but inexhaustible research of the final landing of our serenity.

To try to answer these questions, scientists must work within the field of their competence without forgetting the scientific rigor, accompanied by an unyielding ethic that can make their work more humanized and above all always the use of wisdom, avoiding to proceed with blinkers.

\section{Introduction}

We know that the total, limited, amount of power of the Universe is: $\mathrm{L}_{\max } \sim \varepsilon_{\mathrm{Pl}} / \mathrm{t}_{\mathrm{Pl}} \sim c^{5} / \mathrm{G} \sim 3.6 \times 10^{59} \mathrm{erg} \mathrm{s}^{-1}$

where $\varepsilon_{\mathrm{Pl}}$ and $t_{\mathrm{Pl}}$ are the Planck energy and time, $\mathrm{G}$ is the gravitational constant, and $\mathrm{c}$ is the light velocity.

Then we can say that the Universe is permeated by energy in a continuous exchange with matter according to the famous Einstein law $\mathrm{E}=\mathrm{mc}^{2}$. The Universe is a living being.

The Bridge between the Big Bang and Biology undoubtedly exists, as discussed in the book edited by Giovannelli (2001a). The big problem is how to cross this bridge, and the main question is: what are the experimental tools for understanding the pillars of this Bridge?

In order to cross this bridge, as always when we cross a bridge, we MUST advance slowly, step by step, with continuity, because everything is smoothly linked in the "magma" of the Universe, from the infinitely small to infinitely big, as sketched in Fig. 2 (Rees, 1988a) "Origins. The Darwin College lectures (1986)" (Fabian, 1988).

Indeed, if we look at the Fig. 3 (upper left panel) - where a section of the metabolic network of a "simple" bacterium is shown - we can note that each point (each chemical compound) is connected to any other point through the complexity of the network (Luisi \& Capra, 2014) exactly the same occurring in the "cosmic network" where each point is connected to any other point through the complexity of the network as shown in Fig. 3 (upper right panel) (https://it.wikipedia.org/wiki/ Cosmologia del plasma). The large-scale structure of the Universe, as traced by the distribution of galaxies, is now being revealed by large-volume cosmological surveys. The structure is characterized by galaxies distributed along filaments, the filaments connecting in turn to form a percolating network. Shandarin, Habib \& Heitmann (2010) objective was to quantitatively specify the underlying mechanisms that drive the formation of the cosmic network. By combining percolation-based analyzes with N-body simulations of gravitational structure formation, they elucidate how the network has its origin in the properties of the initial density field (nature) and how its contrast is then amplified by the nonlinear mapping induced by the gravitational instability (nurture). Figure 3 


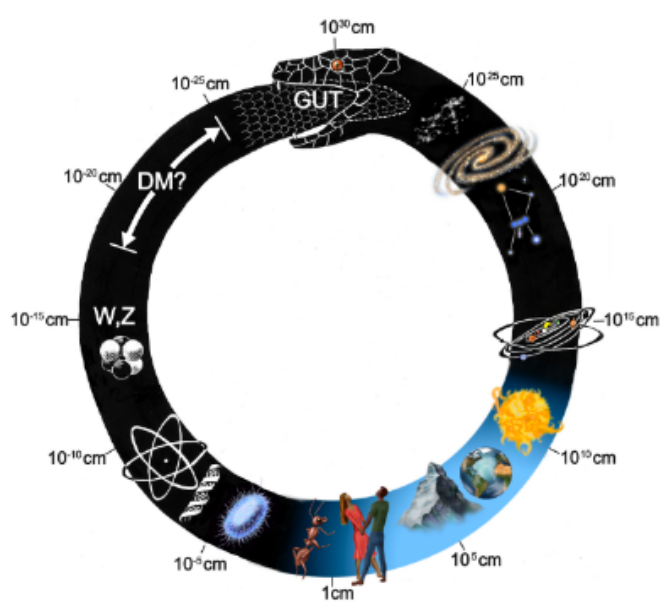

Figure 2: From the infinitely small to infinitely big (adopted by Rees, 1988a).

(lower left panel) shows the human body network: all the organs interact with each other for a good functioning of the body (Luisi \& Capra, 2014). Figure 3 (lower right panel) shows the human society network: every human activity is closely related to all the others (Luisi \& Capra, 2014).

All the components of the Universe follow the same cycle: birth, growth, aging, death as the living beings. Therefore for a complete understanding of the history of the Universe it is necessary to search along that cycle. For this purpose it is mandatory the use of wisdom in Physics, and not only in Physics.

As Albert Einstein affirmed, we can't solve problems by using the same kind of thinking we used when we created them. We can add something more, by using the wisdom: we can attach each kind of problem in a way as general as possible, and in any case it is necessary to go on without blinkers.

\subsection{Accretion Processes in Cosmic Sources}

Accretion is a universal phenomenon that takes place in the vast majority of astrophysical objects. The progress of ground-based and space-borne observational facilities has resulted in the great amount of information on various accreting astrophysical objects, collected within the last decades. The accretion is accompanied by the process of extensive energy release that takes place on the surface of an accreting object and in various gaseous envelopes, accretion disk, jets and other elements of the flow pattern. The results of observations inspired the intensive development of accretion theory, which, in turn, enabled us to study unique properties of accreting objects and physical conditions in the surrounding environment. One of the most interesting outcomes of this intensive study is the fact that accretion processes are, in a sense, self-similar on various spatial scales from planetary systems to galaxies.

This fact gives us new opportunities to investigate objects that, by various reasons, are not available for direct study.

Cataclysmic variable stars are unique natural laboratories where one can conduct the detailed observational study of accretion processes and accretion disks. Indeed, among the cosmic systems where accretion processes occur, undoubtedly, non-magnetic CVs, intermediate polars and polars 

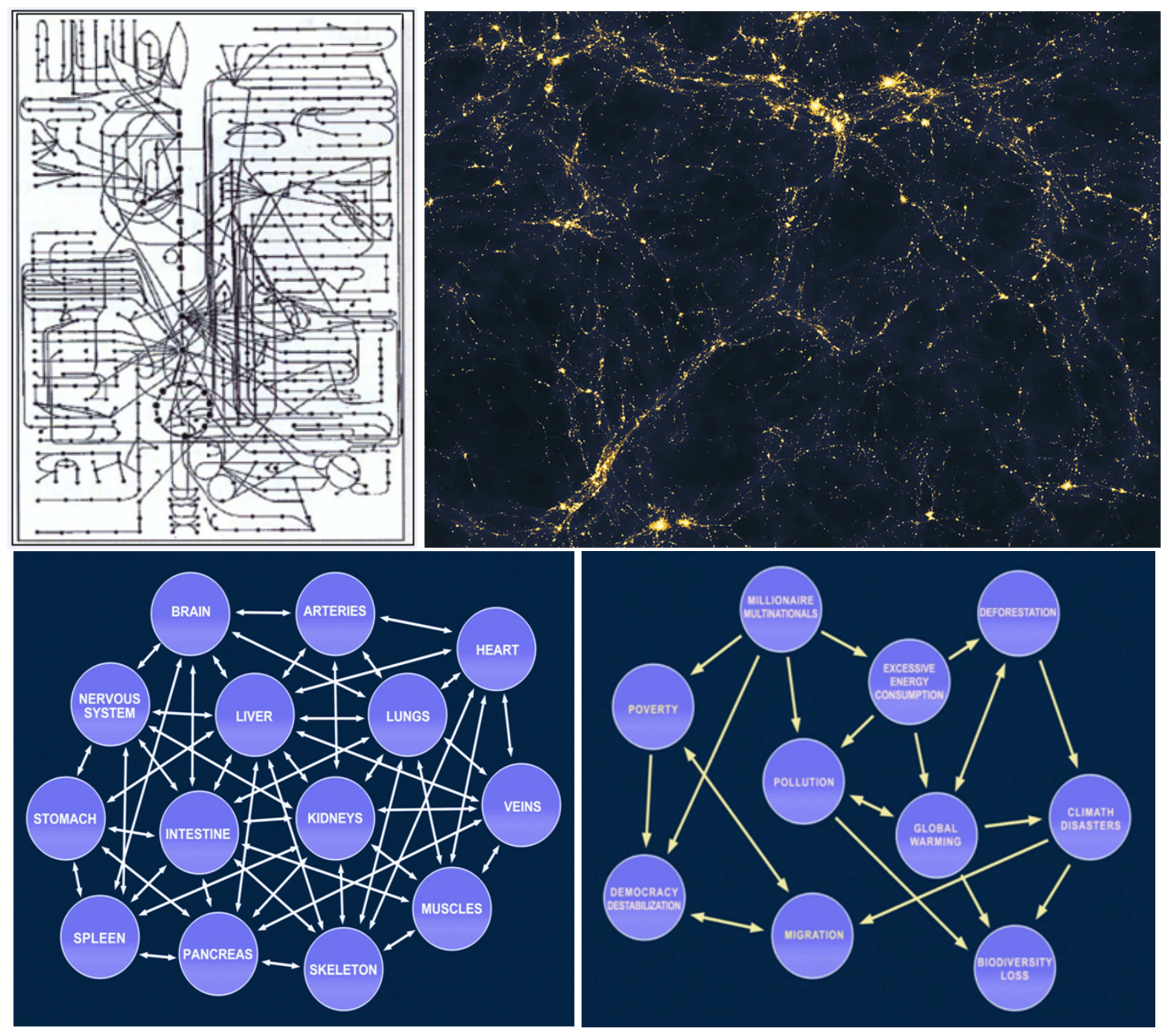

Figure 3: Upper left panel: Section of the metabolic network of a "simple" bacterium (Luisi \& Capra, 2014). Upper right panel: the "cosmic network" (https://it.wikipedia.org/wiki/Cosmologia del plasma). Lower left panel: the human body network. Lower right panel: the human society network (Luisi \& Capra, 2014).

constitute the most powerful probe to test our theories of the various modes of accretion. The reason is rather simple: CVs are enough close to us and their processes develop in time-scales relatively easy to be followed and enough energetic to be easily detected. The long term evolution of CV systems accreting at a prohibitive rate has become a hot topic both in terms of the fate of such systems (all sorts of supernovae) and the microphysics of Eddington and super Eddington mass accretion and mass loss flows. In particular we stress one of the hottest topics in present day astrophysics, namely the progenitors of SN-Ia. This problem is connected with fundamental issues in cosmology. Novae and recurrent novae are the most promising progenitor candidates but so far could not be nailed down.

Figure 4 shows a sketch of cosmic systems where accretion processes occur (Giovannelli \& Sabau-Graziati, 2016, after Scaringi, 2015). 


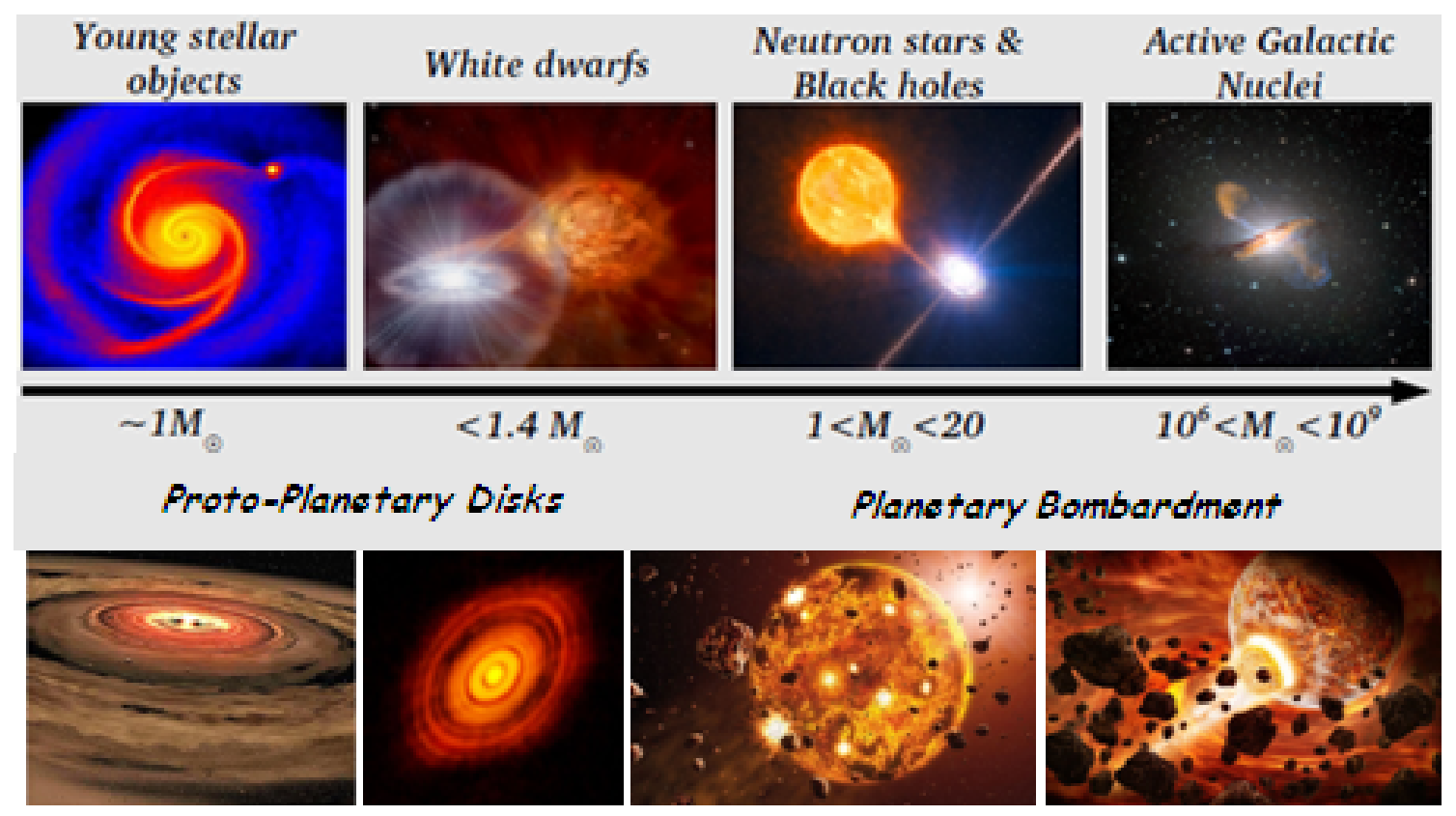

Figure 4: Accretion processes in different cosmic sources (adopted from Giovannelli \& Sabau-Graziati, 2016, after Scaringi, 2015).

\subsection{How the Accretion Theories Developed}

It is very useful to remind some historical remarks in order to paint the developments of the theories that allowed to understand most of the accretion phenomena in cosmic sources. When the first theories about accretion disks around compact stars started to be developed around the 1960-ies, the class of the so-called CVs started to have a leading position in astrophysics. They constituted the perfect laboratories for testing those theories. When the UV window to the universe was opened at the end of 1970-ies with the advent of the historical IUE (International Ultraviolet Explorer), CVs became really one of the most interesting class of objects of the whole astrophysics. Previously, essentially two schools of thought born in Cambridge (UK) and in Warsaw (Poland) in order to tackle with the difficult subject of the mass exchange in close binary systems (e.g. Smak, 1962, 1972, 1981; Paczynski, 1965, 1977; Bath, 1969, 1975, 1976, 1978, 1980, 1984 and the references therein; Bath et al., 1974; Mantle \& Bath, 1983). However, two fundamental papers about accretion disks appeared at the beginning of 1970-ies (Shakura, 1972, Shakura \& Sunyaev, 1973). These papers marked substantially the development of theories about accretion disks around compact objects in binary systems, until present times. Pringle (1981) reviewed accretion disks in astrophysics. In my opinion, the paper by Shakura (1972) (Disk model of gas accretion on a relativistic star in a close binary system) posed the fundamental pillars of the theory of accretion disk, by introducing the $\alpha$ parametrization of the turbulent viscosity.

Among the many papers and books devoted to accretion processes is timely to remind those by Lewin \& van den Heuvel (1983): Accretion-Driven Stellar X-ray Sources, by Frank, King \& Raine (1985): Accretion Power in Astrophysics, and a very interesting tutorial paper by Israel (1996): Accretion Driven $X$-ray Sources where it is possible to find in a clear way the basics of accretion. 


\subsection{Disk observations}

Circumstellar disks can be detected by several techniques, each tracing specific features, temperatures and/or regions of the disk. Combining this knowledge enables us not only to proof the existence of disks but also to probe the physics which is happening inside of them. Most of the disk mass is expected to reside in gas (because disks are made out of interstellar material), however this component is the hardest to detect as it is often frozen out, especially in the outer, cold parts of the disk and in the mid-plane. The disk geometry and the according spectral energy distribution (SED) is schematically shown in Fig. 5. In fact this IR excess was the first observational evidence for disks around young stars (e.g., Strom et al. 1989a; Beckwith et al. 1990).

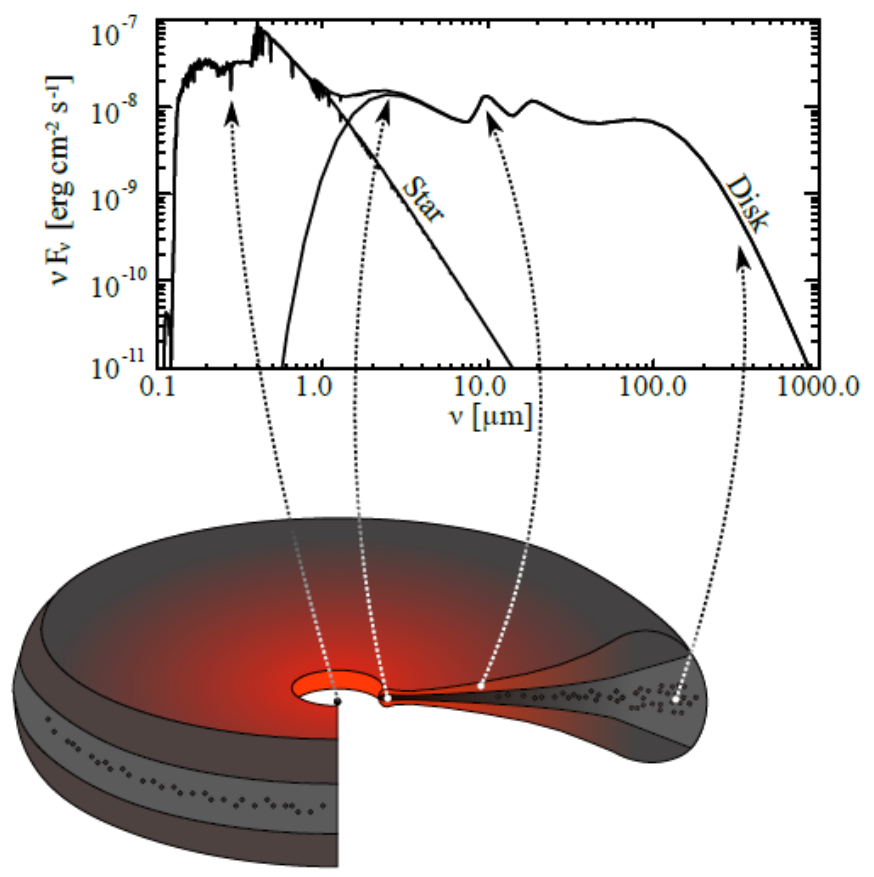

Figure 5: Origin of the spectral energy distribution: at larger wavelength, the disk emission outweighs the stellar spectrum. Generally, the shorter wavelength emission comes from hotter, inner regions of the disk. Large wavelength (submillimeter) probe the dust mass in the outer, optically thin regions (adopted from Birnstiel, 2010, after from Dullemond et al., 2007).

Wolf et al. (2012) present a review of the interplay between the evolution of circumstellar disks and the formation of planets, both from the perspective of theoretical models and dedicated observations. They discuss fundamental questions concerning the formation and evolution of circumstellar disks and planets which can be addressed in the near future with optical and IR long-baseline interferometers, as well as the importance of complementary observations with longbaseline (sub)millimiter interferometers and high-sensitivity IR observations.

How do we know that disks exist? We have many inferences and proofs, like shown in Fig. 6 (after Beckwith, 1998).

Figure 7 shows the SED of XX Cha in which the IR excess clearly demonstrates the presence of an optically-thick, geometrically-thin circumstellar disk (Beckwith, 1998, 1999 after Adams, Lada \& Shu, 1988). 


\section{How do we know disks exist?}
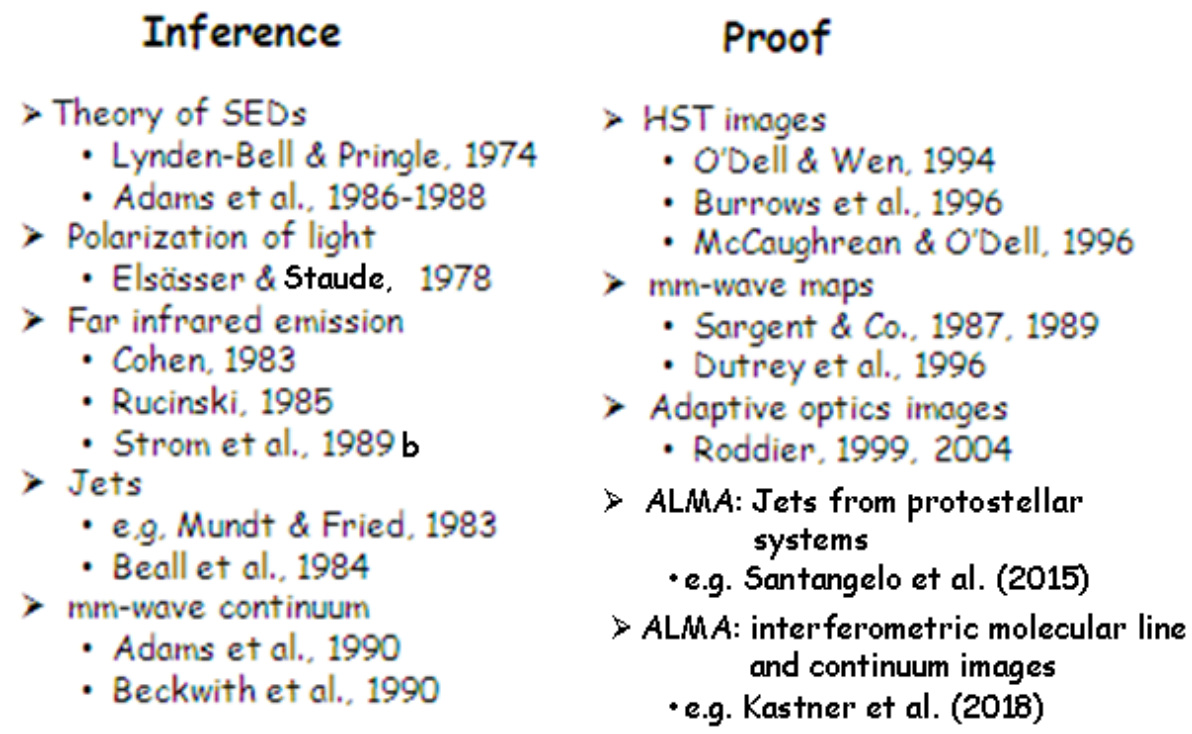

Figure 6: Inferences and proofs for the existence of disks (after Beckwith, 1998).

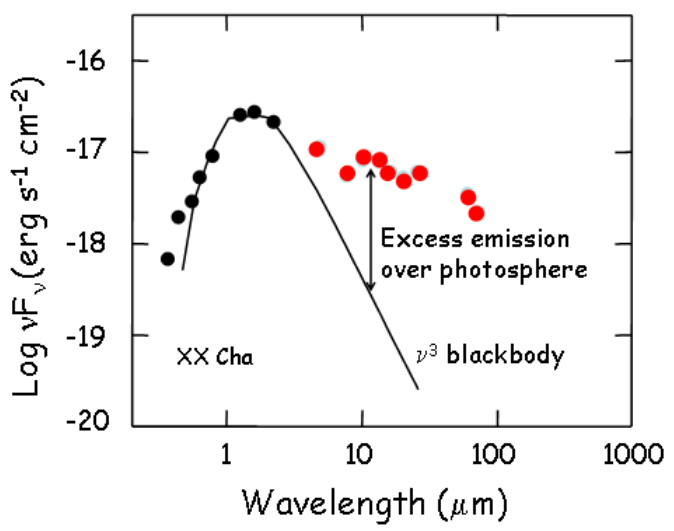

Figure 7: Spectral Energy Distribution of XX Cha (Beckwith, 1998, 1999 after Adams, Lada \& Shu, 1988).

Updated Catalog of Circumstellar Disks can be found at http://www.circumstellardisks.org/ (Created by Caer McCabe. Redesigned by Isabelle H. Jansen. Maintained by Karl Stapelfeldt. Last updated April 29, 2016). The total number of resolved disks is 170, the PMS disks are 129, and the debris disks are 41.

The realization of the Atacama Large Millimeter/submillimeter Array (ALMA) between Europe, North America and Japan started at the end of 2003, after a series of tests, when the first stone was placed in what was to become the most ambitious radio observatory on Earth.

With ALMA it has been possible to study the problem of disk formation: the initial chemical and physical properties of disks are important for the subsequent evolution and the formation of planetary systems. The size and properties of disks as they are assembled are key properties that can be investigated with ALMA at high angular resolution (e.g. Sakai et al., 2014; Aso et 
al., 2015). Detailed observations of young protostellar systems with ALMA have revealed the chemical signatures of the shock at the interface between the infalling envelope and the forming protoplanetary disk. The drastic change of the chemistry at the interface between the envelope and disk are believed to be caused by localized heating at the centrifugal barrier.

ALMA sensitivity allows for the first time true demographical studies of the disk properties in nearby star forming regions, and detailed studies of the chemical complexity of protoplanetary disks (Ansdell et al., 2016).

ALMA spectral line observations of disks in various star forming regions show a deficit in the luminosity of $\mathrm{CO}$ and its isotopologues when compared to the dust continuum luminosity. These observations suggest that disks have a very low gas to dust ratio (3-30 by mass (Miotello et al., 2016).

One of the most stunning results from ALMA have been the discovery of gaps and asymmetries in the dust distribution in planet forming disks. It is still unclear whether a phase transition of the major volatiles are responsible for the symmetric gaps and bright rings in the continuum images of disks, or if the effects of photoevaporation of the disk are dominating, or whether planetesimals, planetary cores and gas giants are responsible. In some cases the presence of planets seems unavoidable, especially in some of the so-called Transition Disks (Qi et al., 2013; Kataoka et al., 2016a; ALMA Partnership et al., 2015; Andrews et al., 2016; Cieza et al. 2016; Isella \& Turner, 2016; Pérez et al., 2016; Walsh et al. 2016).

ALMA has detected molecular gas in several debris disks, despite the fact that molecular gas as expected to be short lived in these systems. The currently favored explanation is that fresh gaseous material is constantly replenished in these systems through shattering of icy bodies. In particular, the observations of the nearby young star Beta Pictoris have revealed an asymmetric distribution of gas with most of the emission associated with a clump of gas. The mass and location of the molecular gas is consistent with being produced by the recent destruction of an icy body with a mass similar to Mars (Dent et al., 2014).

\section{Accretion in Young Stellar Objects}

The study of Pre-Main Sequence Stars (PMSSs) is of great interest as it provides crucial information on stellar evolution and, particularly, on the role of magnetic fields, angular momenta, accretion of matter, and mass loss processes, as well as indirect information on the formation processes of the Sun and Solar system.

Before the space era, practically all observations of PMSSs were only restricted at the optical and near-IR (NIR) spectral regions. With the nowdays knowledge of PMSSs, we can say that they are sites of a variety of extraordinary astrophysical processes, including accretion disks and outflows. The presence of an accretion disk have been strengthened by the observation of nearly Keplerian differential rotation (Hartmann \& Kenyon, 1987a,b) in the optical and NIR spectra of FU Ori, a young stellar object (YSO) whose spectral energy distribution (SED) can be explained by the presence of a disk.

PMS is an important evolutionary phase of all stars, which are evolving from their birth to the Main Sequence (MS), and then to their ultimate fate. A first important difference among stars is due to their masses (or luminosity). Indeed, as well known, high mass stars evolve rapidly into the 
MS and therefore the experimental study of their evolution within the PMS phase is very difficult, due to the lack of a good statistics. On the contrary, low mass stars, because of their slower evolution towards the MS, can be studied in details since it is possible to find large samples of objects belonging to different stages of the PMS evolutionary phase (for details see e.g. Giovannelli, 1994).

The evolution of YSOs has four different phases: Class 0, Class I, Class II and Class III. Class 0 is observable only in submm range. Class I, II and III sources are characterized with a blackbody radiation and an infrared excess. The amount of infrared excess is decreasing during the evolution. Class II and III objects are also visible in the optical bands (see Fig. 8).

Class 0 objects are sources with a central protostar that are extremely faint in the optical and near-IR and that have a significant submillimeter luminosity. Class I sources (a $>0)$ are relatively evolved embedded sources with circumstellar disks and envelopes, they are optically invisible stars

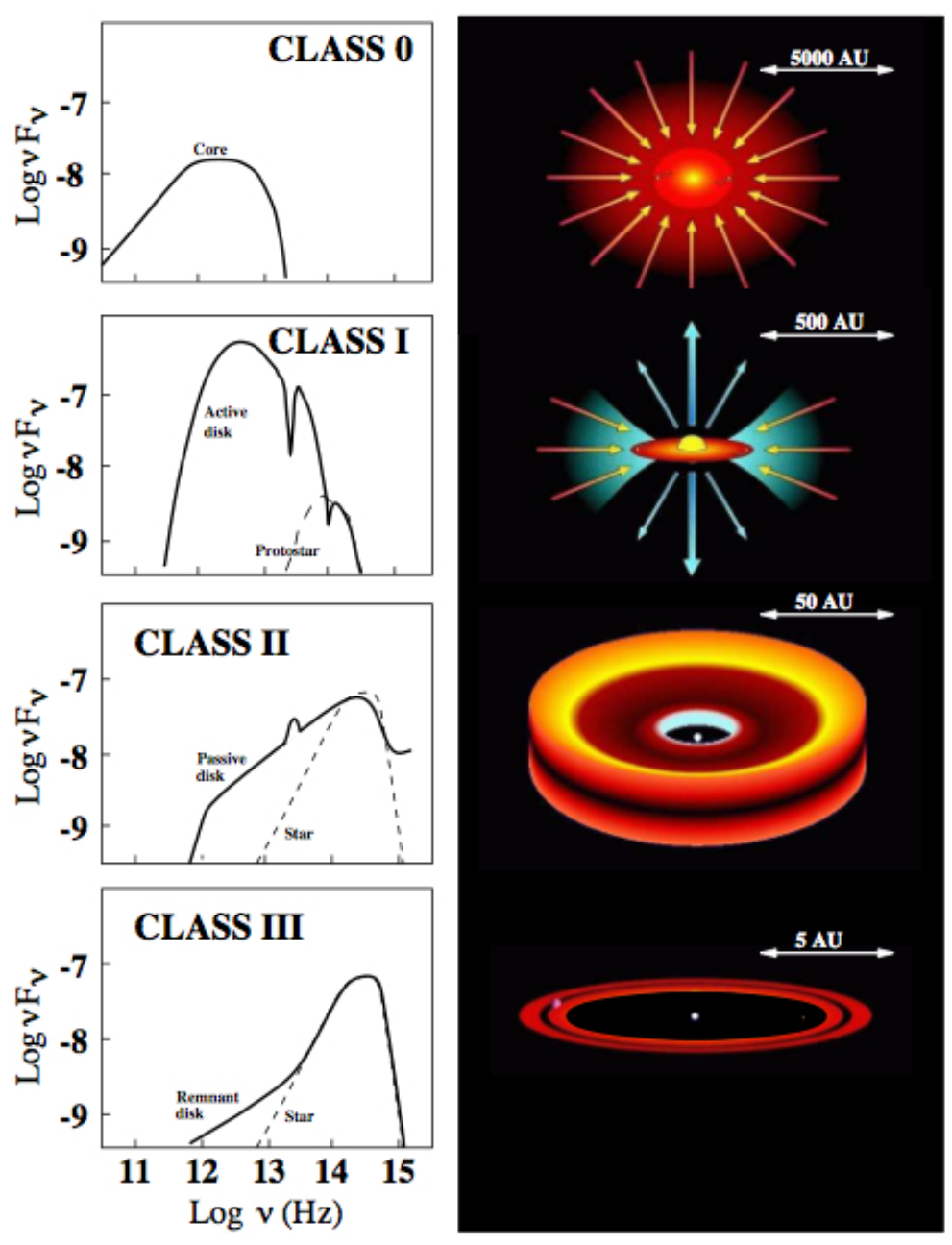

Figure 8: Sketch of the formation of a single star and the evolution of the circumstellar material. The left panels show the SED of the system at different evolutionary stages. The right panels show the corresponding system geometry (Giovannelli \& Sabau-Graziati (2016), adopted from Andrea Isella, 2006, after André, 1994). 
with SEDs that peak at mid-IR to far-IR wavelengths. Class II, or classical T Tauri stars $(-2<\mathrm{a}$ $<0$ ) have significant circumstellar disks, strong emission lines and substantial IR or UV excesses characterized them. Class III, weak emission T Tauri stars $(\mathrm{a}<-2)$ have weak or no emission lines and negligible excesses, they are no longer accreting significant amounts of matter.

It is important to remark the smooth evolution from one class to another. We move from one class to another continuously.

Stellar properties of embedded protostars have been reviewed by White et al. (2007) starting from the objective fact that protostars are precursors to the nearly fully assembled T-Tauri and Herbig Ae/Be type stars undergoing quasi-static contraction towards the zero-age main sequence; they are in the process of acquiring the majority of their stellar mass. Although numerous young stars with spatially extended envelope-like structures appear to fit this description, their high extinction has inhibited observers from directly measuring their stellar and accretion properties and confirming that they are in fact in the main phase of mass accretion (i.e., true protostars).

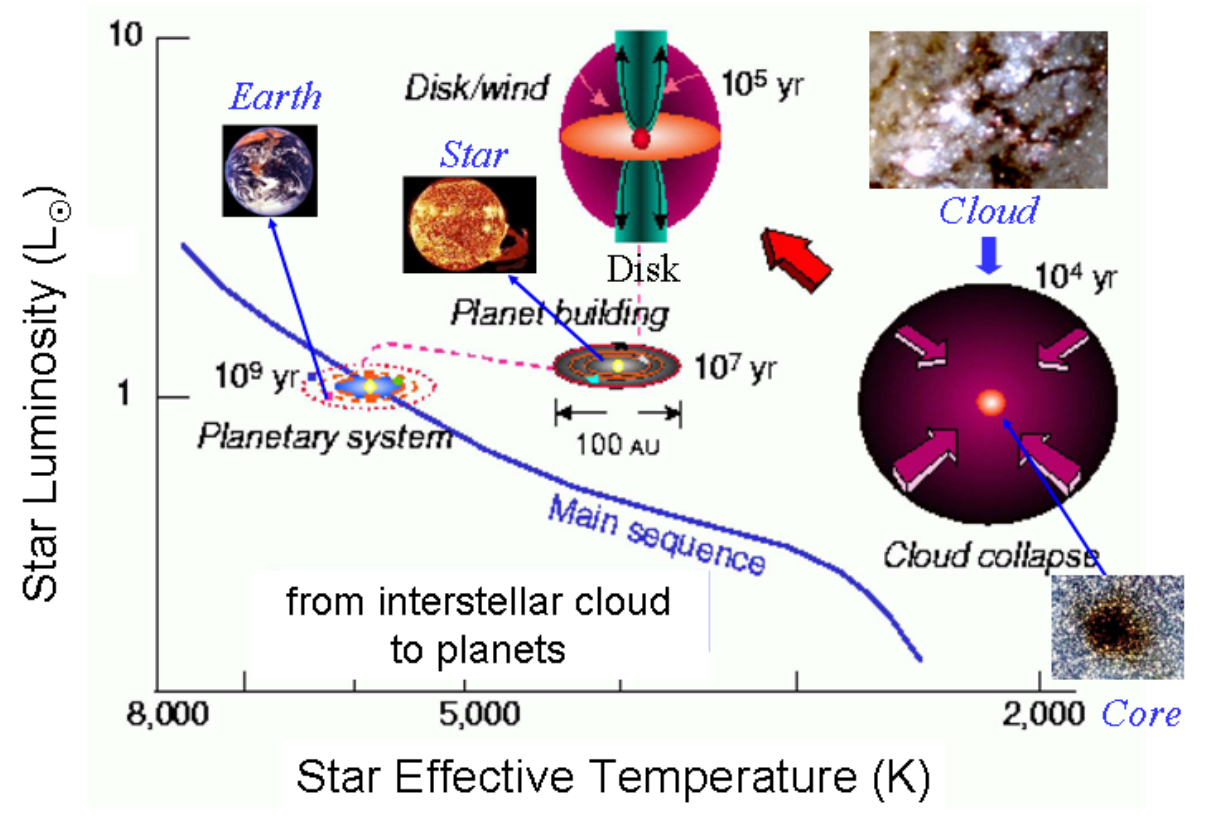

Figure 9: The main stages of planetary formation from the collapse of an interstellar cloud (Giovannelli \& Sabau-Graziati (2016), adapted from Beckwith \& Sargent, 1996).

Figure 9 shows even better the pathway for the formation of planets from an interstellar (IS) cloud in the case of Solar System (Beckwith \& Sargent, 1996). From the collapse of the IS cloud in a timescale of order $10^{4} \mathrm{yr}$ that forms a core, through the active disk/outflow phase $\left(\approx 10^{5} \mathrm{yr}\right)$, the passive disk/planet formation phase $\left(\approx 10^{7} \mathrm{yr}\right)$, and the existence of a stable planetary system by the time the star reaches the MS $\left(\approx 10^{9} \mathrm{yr}\right)$. The formation of the disk occurs naturally. The disk acts as a reservoir for material with too much initial angular momentum to fall onto the central star. Disks emit and reflect light very well and can be seen to relatively large distances from the central star with the new instrumentation both ground- and space-based in different energy regions.

A discussion about the structure and evolution of pre-main-sequence circumstellar disks can be found in the paper by Isella, Carpenter \& Sargent (2009), and a deep discussion about the 
formation and evolution of planetary systems can be found in the paper by Carpenter et al. (2009) in which they used Spitzer photometric and spectroscopic observations.

\subsection{T Tauri Stars}

Following the paper by Linsky \& Schöller (2015) the evolution of YSOs from dense clumps of interstellar gas and dust to main sequence stars proceeds through several stages (Feigelson \& Montmerle, 1999), as shown in Fig. 8. The earliest phase of a protostar (referred to as a Class 0 object) consists of a collapsing massive disk detectable only at millimeter and far-infrared wavelengths. After $\sim 10^{5} \mathrm{yr}$, the collapsing cloud has formed a Class I object consisting of a heavily obscured star heated by the release of gravitational energy and a large circumstellar disk. After $\sim 10^{6} \mathrm{yr}$, the star becomes optically visible above and below the plane of the disk, although the visible spectrum is veiled by emission from the disk. These stars are called Classical T Tauri stars (CTTSs) or Class II objects. Strong UV emission seen in CTTSs results mostly from accretion of gas along magnetic field lines from the disk to the star, producing postshock regions with strongly enhanced emission in the HeI 5876 and other lines. Strong X-ray emission can be produced in the postshock region, closed-field lines in the stellar corona, and where stellar and disk magnetic fields interact. In the last stage of evolution to the main sequence, which occurs between a few million years and $\sim 10^{7} \mathrm{yr}$, most of the gas and dust from the disk has condensed into planets or has been accreted onto the star, which is now called a Weak-lined T Tauri star (WTTS) or Class III object. WTTSs are often called naked TTSs because their ultraviolet emission line spectrum is no longer obscured by a disk.

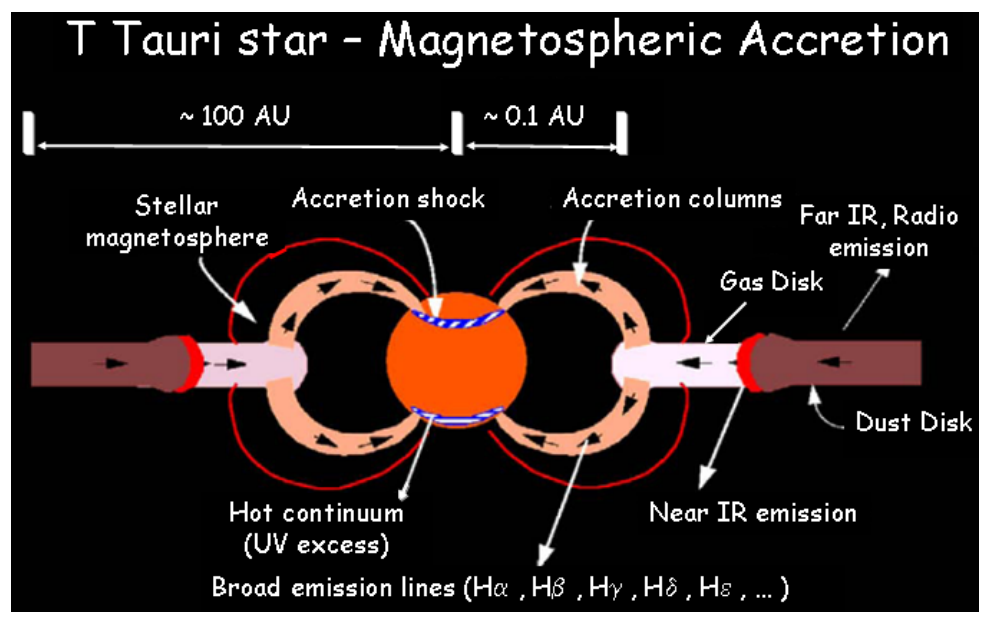

Figure 10: Magnetically funneled accretion stream producing broadened emission lines (Giovannelli \& Sabau-Graziati (2016), after Hartmann, 1998 and Feigelson \& Montmerle, 1999). The dimensions marked are not in scale.

The importance of a deep study of TTSs is based among others because they probably look like the Sun in its very early stages (although with a scaling factor of $\sim 10^{3}-10^{4}$ ). TTSs are subgiant of late spectral type (later than K up to M5.5) and show high variability, strong HI, CaII, FeII emission lines, strong IR excess; the underlying photospheres are relatively cool: $\mathrm{T}<6000$ $\mathrm{K}$ (typically $\mathrm{T} \sim 4000 \mathrm{~K}$ ); masses: $0.5 \mathrm{M}_{\odot} \leq \mathrm{M} \leq 3 \mathrm{M}_{\odot}$; age: $\sim 10^{5}-10^{7} \mathrm{yr}(\mathrm{e}, \mathrm{g}$. Giovannelli, 1994). 
One of the most intriguing problem is connected with the origin of magnetic field. This argument has been deeply discussed by Feigelson \& Montmerle (1999). It is difficult to study YSO magnetic fields directly, and in most cases, indirect tracers of magnetic activity such as cool star spots or high energy radiation produced by violent field reconnection must be used.

After a long multifrequency campaign of observations of the CTTS RU Lupi (Giovanelli et al., 1995) and the subsequent derived model (Lamzin et al., 1996), the proposed model by Feigelson \& Montmerle (1999, after Hartmann, 1998) - shown in Fig. 10 - can be confirmed: an accretion stream magnetically funneled produces broadened emission lines. The different zones interested in the emissions are clearly marked in Fig. 10.

Chiang \& Goldreich (1997) derived hydrostatic, radiative equilibrium models for passive disks surrounding $\mathrm{T}$ Tauri stars. Each disk is encased by an optically thin layer of superheated dust grains. This layer re-emits directly to space about half the stellar energy it absorbs. The other half is emitted inward and regulates the interior temperature of the disk. The heated disk flares. As a consequence, it absorbs more stellar radiation, especially at large radii, than a flat disk would. The portion of the spectral energy distribution contributed by the disk is fairly flat throughout the thermal infrared. At fixed frequency, the contribution from the surface layer exceeds that from the interior by about a factor 3 and is emitted at more than an order of magnitude greater radius. Spectral features from dust grains in the superheated layer appear in emission if the disk is viewed nearly face-on.

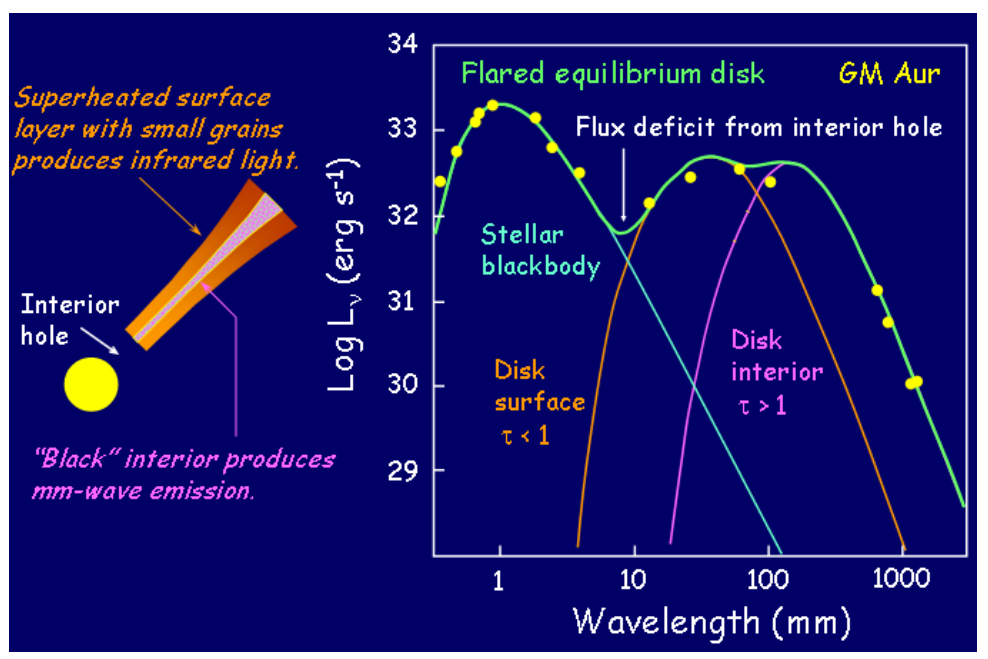

Figure 11: Observed Spectral Energy Distribution of GM Aur, and flared radiative equilibrium disk fit to GM Aur. The contribution of the different component of the system are clearly indicated (Giovannelli \& Sabau-Graziati, 2016, adopted from Beckwith, 1998 after Chiang \& Goldreich, 1997).

Beckwith (1998) derived from the paper by Chiang \& Goldreich (1997) the SED of the T Tauri star GM Aur, reported in Fig. 11, where the contribution of the different part of the system are clearly illustrated.

Material from the thick disk flows along the star's magnetic field lines and is deposited onto the star's surface. When material hits the star, it lights up brightly. In order to measure the interior hole or inner gap in a protoplanetary disk, the technique of "Light Echo" is used to measure the distance from a star to its surrounding protoplanetary disk: the light echo from the disk is delayed 

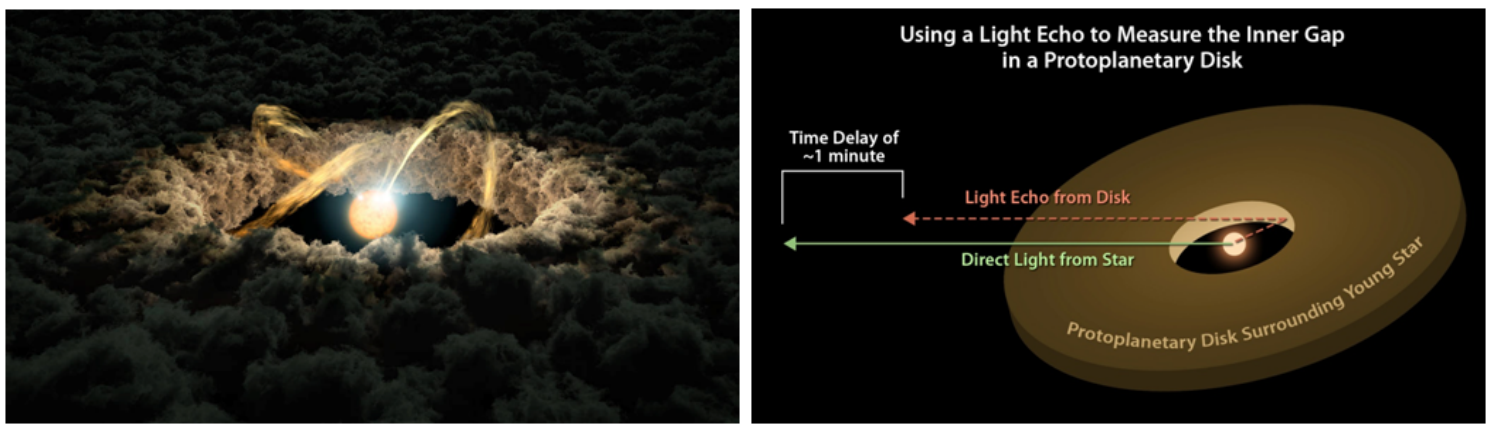

Figure 12: Light Echo technique for meauring the distance from a star to its surrounding protoplanetary disk (Images Credit: NASA/JPL-Caltech).

with respect to the direct light coming from the star. Figure 12 shows in the left panel an artistic picture of the inner gap, and in the right panel the "Light Echo" technique used for measuring the gap (Images Credit: NASA/JPL-Caltech).

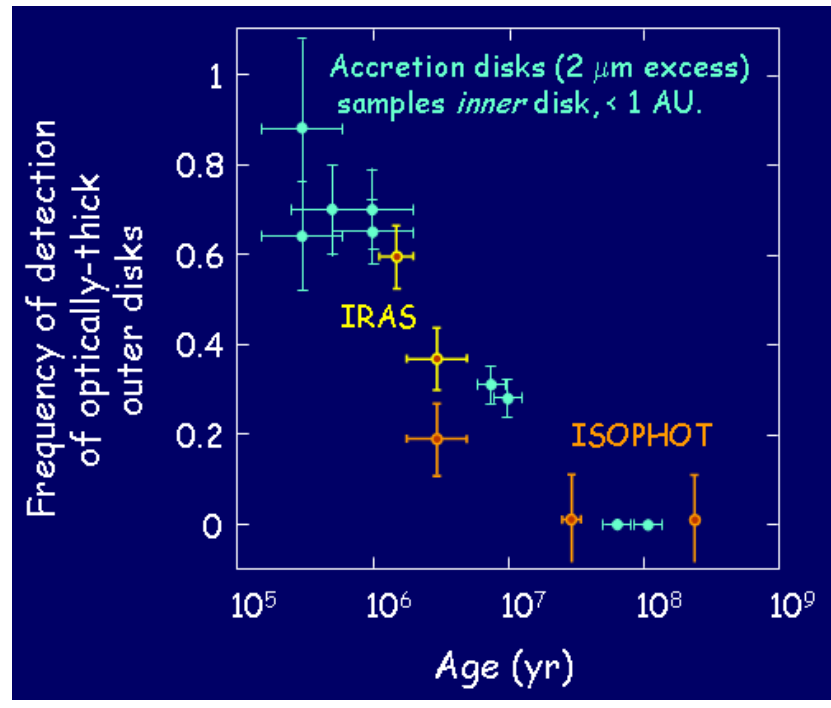

Figure 13: Frequency of detections as a function of cluster age for several star-forming regions observed by ISO, IRAS, and ISOPHOT (Giovannelli \& Sabau-Graziati, 2016, adapted from Beckwith, 1998 after Meyer \& Beckwith, 2000).

While it appears that most young stars form accompanied by a circumstellar disk, it is not clear that all disks form planets. Several factors could play a crucial role in determining the fate of an accretion disk. It has been suggested that disks evolve more quickly around high mass stars $\left(\mathrm{M}_{\star}>\right.$ $1.0 \mathrm{M}_{\odot}$ ) compared to low mass stars (Natta, Grinnin \& Mannings, 2000). Meyer \& Beckwith (2000) reviewed the observed correlations of disk properties with time as studied from ISO, IRAS, ISOPHOT. Figure 13 shows the frequency of detections of optically-thick disks as a function of cluster age for several star-forming regions observed. It appears that optically-thick disks dissipate or coagulate into larger bodies on a timescale comparable to the termination of the main accretion phase in T Tauri disks (Giovannelli \& Sabau-Graziati, 2016, adapted from Beckwith, 1998 after Meyer \& Beckwith, 2000). 
Dust opacities are relevant for dense regions in molecular clouds and for protoplanetary accretion disks. This problem was discussed by Henning, Michel \& Stognienko (1995), and the knowledge of opacities is important in order to settle the controversy among models. An important contribution will be given by the MILLIMETRON Space Observatory (Smirnov et al., 2012; Kardashev et al., 2014) which will operate in a region $(\sim 0.02-20 \mathrm{~mm})$, useful for giving answers to the uncertain values of insterstellar opacities, as shown in the Fig. 14 (light brown ellipse).

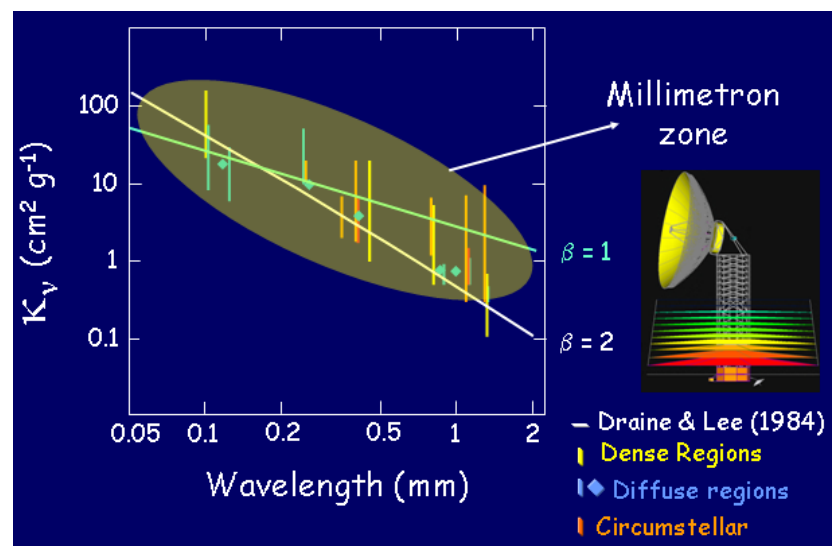

Figure 14: Uncertainties on interstellar opacities. The light brown ellipse mark the zone covered by the Millimetron (Giovannelli \& Sabau-Graziati, 2016, after Beckwith, 1998 and Giovannelli \& Sabau-Graziati, 2010).

With the advent of ALMA (The Atacama Large Millimeter/submillimeter Array) - a worldwide collaboration (ESO/NAOJ/NRAO) - (ALMA Science Advisory Committee, 2015; Carpenter et al., 2018) unbelievable advancement have been obtained in many fields of astrophysics, and in particular in that of protoplanetary science. Figure 15 (left panel) shows an image of the protoplanetary disk around HL Tauri - a T Tauri star - (Credit: ALMA (ESO/NAOJ/NRAO), NSF, 2014; Brogan et al. - ALMA Partnership -, 2015). Figure 15 (right panel) shows the model (Dong, Zhu \& Whitney, 2015).

This is the sharpest image ever taken by ALMA - sharper than is routinely achieved in visible light with the NASA/ESA Hubble Space Telescope. It shows the protoplanetary disk surrounding the young star HL Tauri. These new ALMA observations reveal substructures within the disk that have never been seen before and even show the possible positions of planets forming in the dark patches within the system (Brogan et al. - ALMA Partnership -, 2015). At a resolution of 35 $\mathrm{AU}$, they resolve the molecular component of the disk in $\mathrm{HCO}^{+}(1-0)$ which exhibits a pattern over Local Standard of Rest (LSR) velocities from 2-12 $\mathrm{km} \mathrm{s}^{-1}$ consistent with Keplerian motion around $\mathrm{a} \sim 1.3 \mathrm{M}_{\odot}$ star. The model developed by Dong, Zhu \& Whitney (2015) fits very well the observation. This means that we can totally rely on the models developed for protoplanetary disks. The confirmation is shown in Fig. 16 where a certain number of experimental data are compared with the models (Antoniucci, 2018).

These new results represent an enormous step forward in the understanding of how protoplanetary disks develop and how planets form.

The circumstellar environments of classical T Tauri stars are challenging to directly image because of their high star-to-disk contrast ratio. One method to overcome this is by using imaging 


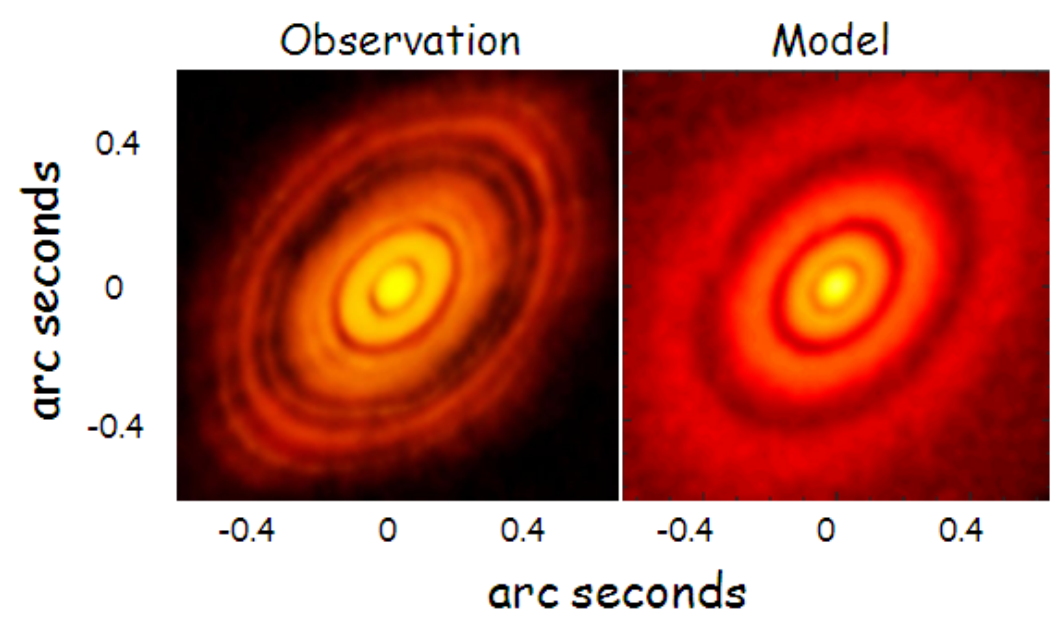

Figure 15: Left panel: the protoplanetary disk around the $\mathrm{T}$ Tauri star HL Tauri (Credit: ALMA (ESO/NAOJ/NRAO), NSF, 2014; Brogan et al. - ALMA Partnership -, 2015). Right panel: the model of the protoplanetary disk around the T Tauri star HL Tauri (Dong, Zhu \& Whitney, 2015).
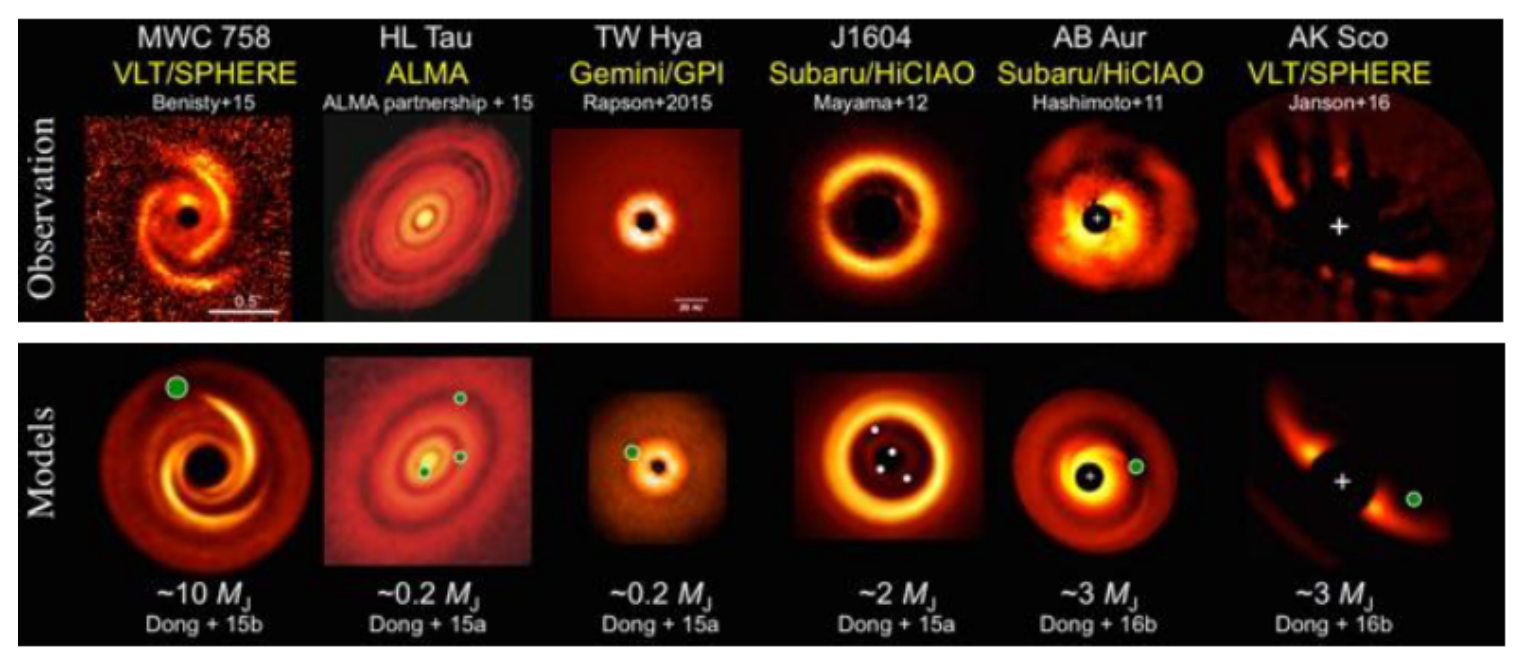

Figure 16: Upper panel: Observations of several systems. Lower panel: Models for the corresponding systems (courtesy of Simone Antoniucci, 2018).

polarimetry where scattered and consequently polarized starlight from the star's circumstellar disk can be separated from the unpolarized light of the central star.

Jeffers et al. (2014) performed a campaign of observations to measure the complete linear polarization signal (Stokes $\mathrm{Q}$ and $\mathrm{U}$ ). The data-reduction software has specifically been developed for ExPo with the aim of minimizing atmospheric and instrumental effects. ExPo is a sensitive imaging-polarimeter is a regular visitor instrument at the Nasmyth focus of the $4.2 \mathrm{~m}$ William Herschel Telescope (Rodenhuis, Sprenger \& Keller, 2012).

Jeffers et al. (2014) present images of the circumstellar environment of SU Aur, a classical T Tauri star at the transition of T Tauri to Herbig stars. SU Aur's disk was modeled using radiativetransfer models to fit the SED (Spectral Energy Distribution), by using the following parameters: 

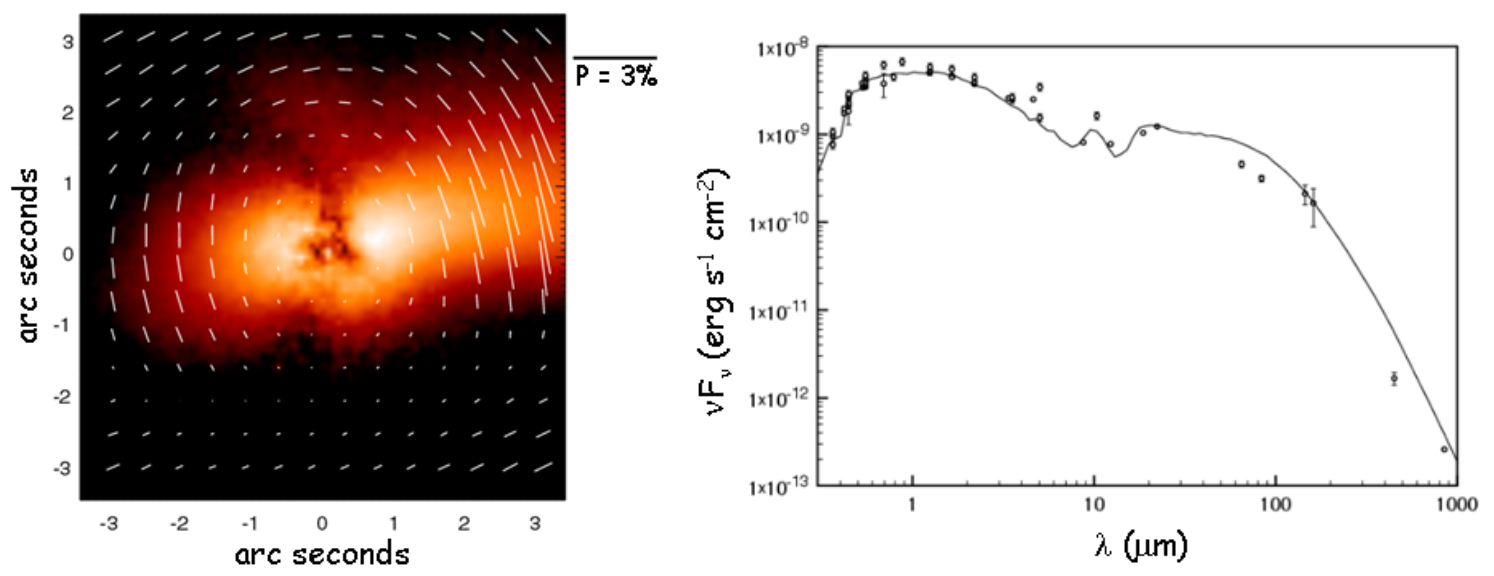

Figure 17: Left panel: ExPo image of the circumstellar environment of SU Aur in polarised intensity, taken without a filter. The intensity scale is logarithmic over two orders of magnitude and the length of the vectors represents a lower limit to the true polarization degree. North is up and east is to the left. Right panel: the SED of SU Aur (adopted from Jeffers et al., 2014).

$\mathrm{M}_{\star}=1.88 \mathrm{M}_{\odot}, \mathrm{d}=143 \mathrm{pc}, \dot{\mathrm{M}}=3 \times 10^{-7} \mathrm{M}_{\odot} \mathrm{yr}^{-1}$, the computed total dust mass $1.2 \times 10^{-4} \mathrm{M}_{\odot}$, which is $\approx 1 \%$ of the stellar mass.

Figure 17 (left panel) shows ExPo image of the circumstellar environment of SU Aur in polarised intensity (PI), taken without a filter. The intensity scale is logarithmic over two orders of magnitude and the length of the vectors represents a lower limit to the true polarisation degree. North is up and east is to the left. Figure 17 (right panel) shows the SED of SU Aur.

The results obtained with the disk model of SU Aur were compared with the ExPo image. Jeffers et al. (2014) found very small grains in the surface layers of the disk. The modeled turbulence parameter is about two orders of magnitude higher than previously determined for Herbig Ae/Be and T Tauri disks, indicating an enhanced level of grain mixing in the disk, though this value needs to be confirmed with more detailed multiwavelength (imaging) studies. In their ExPo images, they additionally resolved a large extended nebulosity that is most likely a remnant of the prenatal molecular cloud because of the position angle of the disk and the brightness of the nebulosity, and is not a cavity carved out by a jet, as previously speculated.

This is one more example of the continuity in nature: Su Aur is in between the phase of Herbig $\mathrm{Ae} / \mathrm{Be}$ and T Tauri stars.

The submillimiter polarization observations of protoplanetary disks is an important tool for determining the size of grains. Indeed, for example, Kataoka et al. (2016a) - studyimg the circumstellar disk around the Herbig Ae/Be star HD 142527 by using the Atacama Large Millimeter/submillimeter Array - conclude that self-scattering of thermal dust emission plays a major role in producing polarization at millimeter wavelengths in this protoplanetary disk. Also, this puts a constraint on the maximum grain size to be approximately $150 \mu \mathrm{m}$ if one assumes a compact spherical dust grains. Kataoka et al. (2016b) found grain size constraints on HL Tau by using the millimeter-wave polarization of the protoplanetary disk around HL Tau, which has been interpreted as the emission from elongated dust grains aligned with the magnetic field in the disk. Dust grains are assumed to be spherical and to have a power-law size distribution. They changed the 
maximum grain size with a fixed dust composition in a fixed disk model to find the grain size to reproduce the observed signature. They found that the direction of the polarization vectors and the polarization degree can be explained with the self-scattering. Moreover, the polarization degree can be explained only if the maximum grain size is $\sim 150 \mu \mathrm{m}$. The obtained grain size from the polarization is different from that which has been previously expected from the spectral index of the dust opacity coefficient (a millimeter or larger) if the emission is optically thin.

The growth of dust grains from sub- $\mu \mathrm{m}$ to $\mathrm{mm}$ and $\mathrm{cm}$ sizes is the first step towards the formation of planetesimals. Theoretical models of grain growth predict that dust properties change as a function of disk radius, mass, age, and other physical conditions. High angular resolution observations at several (sub-)mm wavelengths constitute the ideal tool with which to directly probe the bulk of dust grains and to investigate the radial distribution of their properties. Tazzari et al. (2016) lay down the methodology for a multiwavelength analysis of (sub-)mm and cm continuum interferometric observations to self-consistently constrain the disk structure and the radial variation of the dust properties. They analyzed three disks and found a common trend of larger (cm-sized) grains in the inner disks $(\mathrm{R}<30-40 \mathrm{AU})$ and smaller (mm-sized) grains in the outer disks, but different slopes of $\sigma_{\max }(\mathrm{R})$ for different disks. A natural question that arises is whether this is an evolutionary trend (caused by dust growth processes) or an intrinsic variability of disk properties. They cannot answer this question because of the very limited sample, but the analysis method is ready to be performed on larger samples that will have the potential of giving some insight into possible correlations and intrinsic variability.
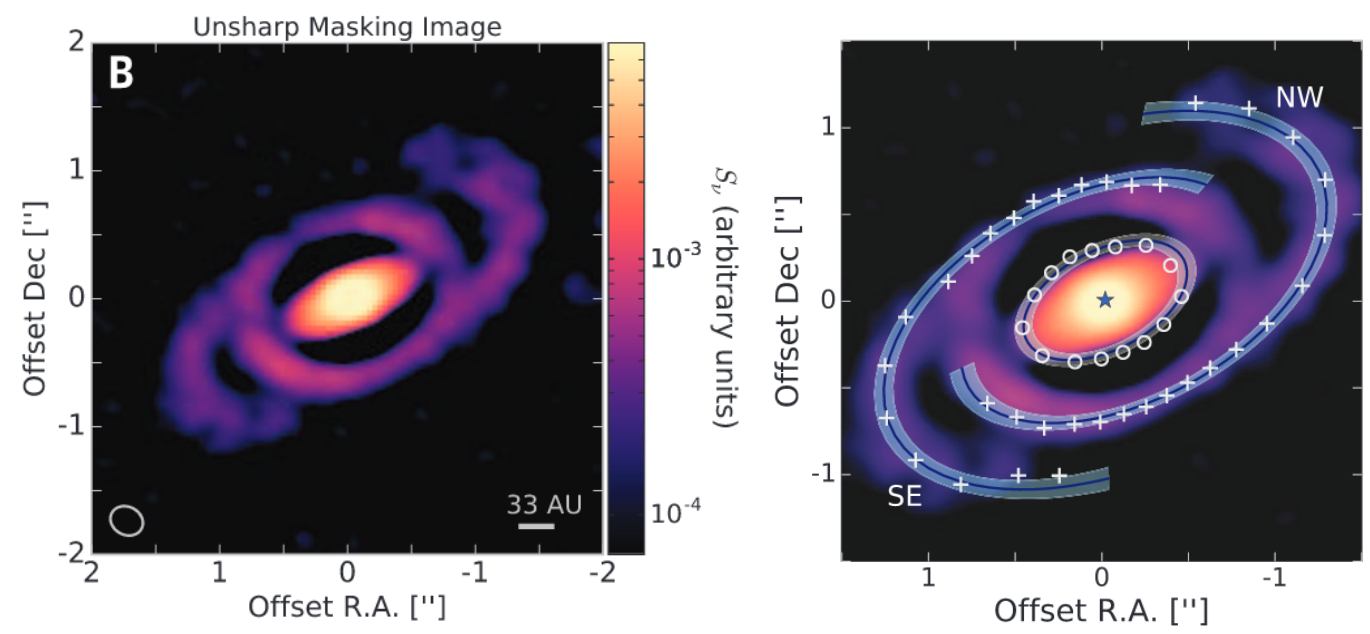

Figure 18: Left panel: the thermal dust emission from the protoplanetary disk surrounding Elias 2-27 with increased contrast image from processing the original ALMA observations with an unsharp masking filter. Right panel: the model of symmetric spirals and dark ring for the Elias 2-27 protoplanetary disk (Peréz et al., 2016).

Gravitational forces are expected to excite spiral density waves in protoplanetary disks, disks of gas and dust orbiting young stars. However, previous observations that showed spiral structure were not able to probe disk midplanes, where most of the mass is concentrated and where planet formation takes place. Using the Atacama Large Millimeter/submillimeter Array, Peréz et al. (2016) detected a pair of trailing symmetric spiral arms in the protoplanetary disk surrounding 
the young star Elias 2-27. The arms extend to the disk outer regions and can be traced down to the midplane. These millimeter-wave observations also reveal an emission gap closer to the star than the spiral arms. They argue that the observed spirals trace shocks of spiral density waves in the midplane of this young disk.

Figure 18 shows i) the thermal dust emission from the protoplanetary disk surrounding Elias 227 with increased contrast image from processing the original ALMA observations with an unsharp masking filter (left panel); ii) the model of symmetric spirals and dark ring for the Elias 2-27 protoplanetary disk (Peréz et al., 2016).

\section{Formation of planets}

Following the very interesting review by Dullemond \& Monnier (2010), to understand how planetary systems form in the dusty disks around pre-main-sequence stars, a detailed knowledge of the structure and evolution of these disks is required. Although this is reasonably well understood for the regions of the disk beyond about $1 \mathrm{AU}$, the structure of these disks inward of $1 \mathrm{AU}$ remains a puzzle. This is partly because it is very difficult to spatially resolve these regions with current telescopes. But it is also because the physics of this region, where the disk becomes so hot that the dust starts to evaporate, is poorly understood.

Figure 19 shows the rich structure of protoplanetary disks, with very different physics playing a role in different regions of the disk.

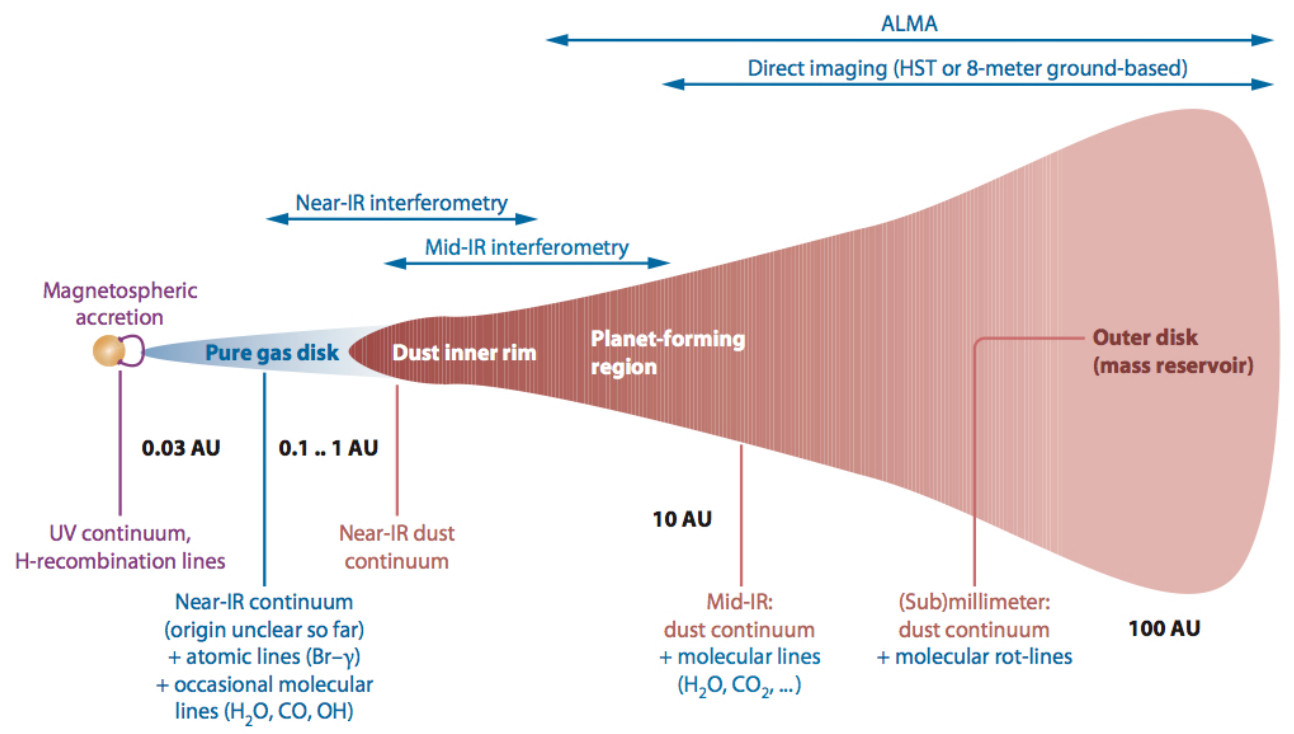

Figure 19: Pictogram of the structure and spatial scales of a protoplanetary disk. Note that the radial scale on the $\mathrm{x}$-axis is not linear. Above the pictogram shows which techniques can spatially resolve which scales. Below shows which kind of emission arises from which parts of the disk (adopted from Dullemond \& Monnier, 2010).

One can see the strikingly large dynamic range that is involved: the outer radius of a protoplanetary disk can be anywhere from a few tens of astronomical units up to 1,000 AU or more, whereas the inner disk radius is typically just a few stellar radii, i.e., of the order of $0.02 \mathrm{AU}$. This spans a 
factor of $10^{4}-10^{5}$ in spatial scale. For each orbit of the outer disk, we have up to ten million orbits of the inner edge of the disk. Equivalently, the dynamic timescale on which various processes in the disk take place is also a million times shorter (i.e., faster) in the very inner disk regions than in the outer disk regions. In addition, the temperatures differ vastly: from $\mathrm{T} \gg 10^{3} \mathrm{~K}$ in the inner disk regions down to $\mathrm{T} \sim 10-30 \mathrm{~K}$ in the outer regions.

This large dynamic range in spatial scale, density, and temperature means that very different observational techniques have to be applied to probe the various regions of these disks. Long wavelength telescopes [in the far-infrared (FIR) and millimeter regime] predominantly probe the outer $\sim 10$ - few 100 AU of disks, whereas mid-IR observations probe intermediate radii about a few AU, near-infrared (NIR) observations probe the inner AU, and finally optical and UV observations typically probe the regions very close to the stellar surface $(\sim 0.01-0.1 \mathrm{AU})$. Of course, due to dust scattering and sometimes due to quantum-heated grains, short wavelength radiation can also be used to probe the outer disk regions, but for thermally emitted radiation from the disk, this rule of thumb applies very well.

Beckwith et al. (1990) from a continuum observations at $1.3 \mathrm{~mm}$ of 86 pre-main-sequence stars in the Taurus-Auriga dark clouds showed that $42 \%$ have detectable emission from small particles. The detected fraction is only slightly smaller for the weak-line and "naked" T Tauri stars than for CTTSs, indicating that the former stars often have circumstellar material. The typical disk has an angular momentum comparable to that generally accepted for the early solar nebula, but very little stored energy, almost five orders of magnitude smaller than that of the central star. Their results demonstrate that disks more massive than the minimum mass of the proto-solar system commonly accompany the birth of solar-mass stars and suggest that planetary systems are common in the Galaxy.

This fact is supported also by the observations of submillimeter flux densities from the circumstellar disks around 29 pre-main-sequence stars (Beckwith \& Sargent, 1991). The properties of these disks are of special interest, because they bear many similarities to those of the primitive solar nebula. Studies of such systems may therefore lead to a better understanding of the formation of planetary systems like our own.

Meyer et al. (2002) discussed the formation and evolution of planetary systems utilizing the sensitivity of SIRTF (the Space Infra-Red Telescope Facility) through the Legacy Science Program to carry out spectrophotometric observations of solar-type stars. They derived circumstellar dust properties around a representative sample of primordial disks (dominated by ISM grains in the process of agglomerating into planetesimals) and debris disks (dominated by collisionally generated dust) over the full range of dust disk optical-depths diagnostic of the major phases of planet system formation and evolution. Hillenbrand (2008) discussed disk-dispersal and planet-formation timescales starting that well before the existence of exo-solar systems was confirmed, it was accepted knowledge that most - if not all - stars possess circumstellar material during the first oneto-several million years of their pre-main sequence lives, and thus that these systems commonly have the potential to form planets.

Following the very interesting paper by Machida, Inutsuka \& Matsumoto (2010) and references therein), we believe that stars are born with a circumstellar disk. The formation of the circumstellar disk is coupled with the angular momentum problem that is a serious problem in the star formation process, and the dynamics of disks may determine the mass accretion rate onto the 
protostar that determines the final stellar mass. In addition, planets are considered to form in the circumstellar (or protoplanetary) disk, and their formation process strongly depends on disk properties such as disk size and mass. Thus, the formation and evolution of the circumstellar disk can provide a significant clue to star and planet formation.

Stars form in molecular clouds that have an angular momentum (Arquilla \& Goldsmith, 1986; Goodman et al., 1993; Caselli, 2002; 2003). Thus, the appearance of a circumstellar disk is a natural consequence of the star formation process when the angular momentum is conserved in the collapsing cloud core. In addition, observations have shown the existence of circumstellar disks around the protostar (e.g., Watson et al., 2007; Dutrey, Guilloteau \& Ho, 2007; Meyer et al., 2007). Numerous observations indicate that circumstellar disks around Class I and II protostars have a size of $\sim 10-1000 \mathrm{AU}$ and a mass of $\sim 10^{-3}-0.1 \mathrm{M}_{\odot}$ (e.g., Calvet, Hartmann \& Strom, 2000; Natta, Grinin \& Mannings, 2000). However, they correspond to phases long after their formation. Because the formation site of the circumstellar disk and protostar are embedded in a dense infalling envelope, we cannot directly observe newborn or very young circumstellar disks (and protostars). Thus, we only observe the circumstellar disks long after their formation, i.e., around the class I or II protostar phase.

Observations also indicate that a younger protostar has a massive circumstellar disk (Natta, Grinin \& Mannings, 2000; Meyer et al., 2007). Enoch et al. (2009) observed a massive disk with $\mathrm{M}_{\text {disk }} \sim 1 \mathrm{M}_{\odot}$ around class 0 sources, indicating that this massive disk can be present early in the main accretion phase. However, unfortunately, observation cannot determine the real sizes of circumstellar disks, and how and when they form. Therefore, we cannot understand the formation process of the circumstellar disk by observations. The theoretical approach and numerical simulation are necessary to investigate the formation and evolution of the circumstellar disk.

For this reason Machida, Inutsuka \& Matsumoto (2010) investigated the formation and evolution of the circumstellar disk in unmagnetized molecular clouds using three-dimensional hydrodynamic simulations from the prestellar core until the end of the main accretion phase. Their calculations indicate that the planet or brown-dwarf mass object may form in the circumstellar disk in the main accretion phase. In addition, the mass accretion rate onto the protostar shows strong time variability that is caused by the perturbation of proto-planets and/or the spiral arms in the circumstellar disk. Such variability provides a useful signature for detecting the planet-sized companion in the circumstellar disk around very young protostars.

Drouart et al. (1999) starting from the fact that each nebula disk is characterized by its initial mass $\mathrm{M}_{\mathrm{D}}$, its initial radius $\mathrm{R}_{\mathrm{D}}$, and the coefficient of turbulent viscosity $\alpha$, they show that these parameters may be constrained by comparing temperature-density profiles to properly chosen physical and chemical Solar System data. They developed an analytical model that permits fruitful comparisons with available Solar System data and helpful constraints on the structure of the primitive solar nebula, as summarized below:

- Theories of the formation of giant planets provide relatively weak constraints on the main parameters which define the nebula, namely the initial mass $M_{D}$, the initial radius $R_{D}$, and the coefficient of turbulent diffusion $\alpha$. However, they conclude that forming the cores of giant planets in a time compatible with the lifetime of the nebula requires a $\alpha$ value equal to at least a few $10^{-4}$. 
- The enrichment in fossil deuterium enrichment in water with respect to the protosolar abundance is by far more constraining. At $t=0, R_{D}$ must be between 8 and $28 \mathrm{AU}, \mathrm{M}_{\mathrm{D}}$ between 0.03 and $0.3 \mathrm{M}_{\odot}$, and $\alpha$ must be between 0.003 and 1 .

- The high viscosity disks they infer $(0.02<\alpha<1)$ are characterized by a MHD turbulence while their low viscosity models $(0.003<\alpha<0.02)$ are charaterized by hydrodynamical turbulence. Other mechanisms susceptible to generating a turbulent nebula compatible with observations seem to be ruled out.

- The strength of magnetic fields calculated from their selected models of nebula is consistent with the remanent magnetism found in carbonaceous chondrites. The lifetime of magnetic fields was short, no more than $10^{5}$ years in the extreme case.

Now we can say that over the past 20 years abundant evidence has emerged that many (if not all) stars are born with circumstellar disks. Understanding the evolution of post-accretion disks can provide strong constraints on theories of planet formation and evolution.

Meyer et al. (2007) reviewed on developments in understanding: i) the evolution of the gas and dust content of circumstellar disks based on observational surveys, highlighting new results from the Spitzer Space Telescope; ii) the physical properties of specific systems as a means to interpret the survey results; iii) theoretical models used to explain the observations; iv) an evolutionary model of our own solar system for comparison to the observations of debris disks around other stars; and v) how these results impact our assessment of whether systems like our own are common or rare compared to the ensemble of normal stars in the disk of the Milky Way.

With a sample of $\sim 328$ stars ranging in age from $\sim 3$ Myr to $\sim 3$ Gyr, Meyer et al. (2006) traced the evolution of circumstellar gas and dust from primordial planet-building stages in young circumstellar disks through to older collisionally generated debris disks. In addition to the observational program, they coordinated a concomitant theoretical effort aimed at understanding the dynamics of circumstellar dust with and without the effects of embedded planets, dust spectral energy distributions, and atomic and molecular gas line emission. Together with the observations, these efforts can provide an astronomical context for understanding whether our solar system - and its habitable planet - is a common or a rare circumstance.

The $2^{\text {nd }}$ JEDI (JEts and Disks at INAF) meeting about Jets, disks and the dawn of planets has been very interesting. The proceedings contain papers about: i) Early Protostellar Phases; ii) Disk Accretion and YSOs; iii) Jets and Winds; iv) Physical and Chemical Properties of Disks (Antoniucci et al., 2015).

Protoplanetary disks dissipate rapidly after the central star forms, on time-scales comparable to those inferred for planet formation. In order to allow the formation of planets, disks must survive the dispersive effects of UV and X-ray photoevaporation for at least a few Myr. Viscous accretion depletes significant amounts of the mass in gas and solids, while photoevaporative flows driven by internal and external irradiation remove most of the gas. A reasonably large fraction of the mass in solids and some gas get incorporated into planets. Gorti et al. (2016) review our current understanding of disk evolution and dispersal, and discuss how these might affect planet formation. They also discuss existing observational constraints on dispersal mechanisms and future directions. In the near future it is desirable to be able to connect disk evolution to planet formation and under- 
stand the close, and perhaps causal, correspondence between timescales for planet formation and disk dispersal.

Recently, investigations about the possible correlation between the mass accretion rate onto the central young star and the mass of the surrounding protoplanetary disk - theoretically predicted - have been performed. For instance, Manara et al. (2016) have accurately and homogeneously determined the photospheric parameters, mass accretion rate, and disk mass for an essentially complete sample of young stars with disks in the Lupus clouds. They found a correlation between the mass accretion rate and the disk dust mass, with a ratio that is roughly consistent with the expected viscous timescale when assuming an interstellar medium gas-to-dust ratio. This confirms that mass accretion rates are related to the properties of the outer disk. We expect in the near future measurements in different clouds in order to better determine such a correlation.

Considering that star and planet formation are the complex outcomes of gravitational collapse and angular momentum transport mediated by protostellar and protoplanetary disks, Kratter \& Lodato (2016) deeply discuss on the role of gravitational instability in this process. They highlight open questions related to the development of a turbulent cascade in thin disks and the role of mode-mode coupling in setting the maximum angular momentum transport rate in thick disks. Gravitational instability provides efficient angular momentum transport in relatively massive protostellar disks. Evidence of this phase observationally remains sparse but is likely to improve in the coming years with more ALMA data. For this purpose it is interesting to look at the paper "The ALMA Development Road Map" (Carpenter et al., 2018).

González-Casanova, Lazarian \& Santos-Lima (2016) consider formation of accretion disks from a realistically turbulent molecular gas using 3D MHD simulations. In particular, they analyze the effect of the fast turbulent reconnection for the removal of magnetic flux from a disk. With their numerical simulations they demonstrate how the fast reconnection enables protostellar disk formation resolving the so-called "magnetic braking catastrophe".

What we can say about the step from disk to planet? Thanks to the unprecedented sensitivity and imaging capabilities offered by the Atacama Large Millimeter Array (ALMA) we have transformed our understanding of protoplanetary disks and, hence, of planet formation. Isella (2016) discuss the main results and caveats related to the measurement of the mass of solids in protoplanetary disks based on millimeter-wave observations. Then he present a recent analysis of the ALMA observations of the HL Tau disk, which suggests that the observed circular rings might be due to the tidal interaction between Saturn mass planets and the circumstellar material. In the conclusion, Isella (2016) argues that the existing observations of protoplanetary disks suggest that planets might form very early on, perhaps at the same time as the formation of the disk itself.

Magnetohydrodynamic(MHD) and photoevaporative winds are thought to play an important role in the evolution and dispersal of planet-forming disks. Recently Fang et al. (2018) report the first high-resolution $\left(\Delta \mathrm{v} \sim 6 \mathrm{~km} \mathrm{~s}^{-1}\right)$ analysis of [SII] $\lambda 4068$, [OI] $\lambda 5577$, and [OI] $\lambda 6300$ lines from a sample of $48 \mathrm{~T}$ Tauri stars. They decompose them into three kinematic components: a highvelocity component (HVC) associated with jets, and a low-velocity narrow (LVC-NC) and broad (LVC-BC) components. They estimate $\dot{\mathrm{M}}_{\text {wind }} / \dot{\mathrm{M}}_{\text {acc }}$ for the LVC and $\dot{\mathrm{M}}_{\mathrm{jet}} / \dot{\mathrm{M}}_{\mathrm{acc}}$ for the HVC. With the likely assumption that the NC wind height is larger than the BC, the LVC-BC $\dot{\mathrm{M}}_{\text {wind }} / \dot{\mathrm{M}}_{\text {acc }}$ is found to be higher than the LVC-NC. These results suggest that most of the mass loss occurs close to the central star, within a few AU, through an MHD driven wind. Depending on the wind height, 
MHD winds might play a major role in the evolution of the disk mass.

\subsection{Habitable Zone in the Milky Way and Exoplanets}

The most important questions about the possible origin of life in our Universe became a real scientific question in the last couple decades when it appeared a near certainty that other planets must orbit other stars. And yet, it could not be proven, until the early 1990's. Then, radio and optical astronomers detected small changes in stellar emission which revealed the presence of first a few, and now many, planetary systems around other stars. We call these planets "exoplanets" to distinguish them from our own solar system neighbors (http://science.nasa.gov/astrophysics/focusareas/exoplanet-exploration/).

We know of over 3,500 confirmed exoplanets, with more than 2,500 of those found in the Kepler data. These planets range in size from larger than Jupiter to smaller than Earth. In just a couple decades, thanks largely to Kepler, we have gone from suspecting exoplanets existed to knowing that there are more exoplanets than stars in our galaxy.

Figure 20 shows the distribution of Kepler planet candidates by size as of July 23, 2015 (Image Credit: NASA Ames/W Stenzel). As we can see there are 955 Earth-like planets in the neighbourhood of solar system.

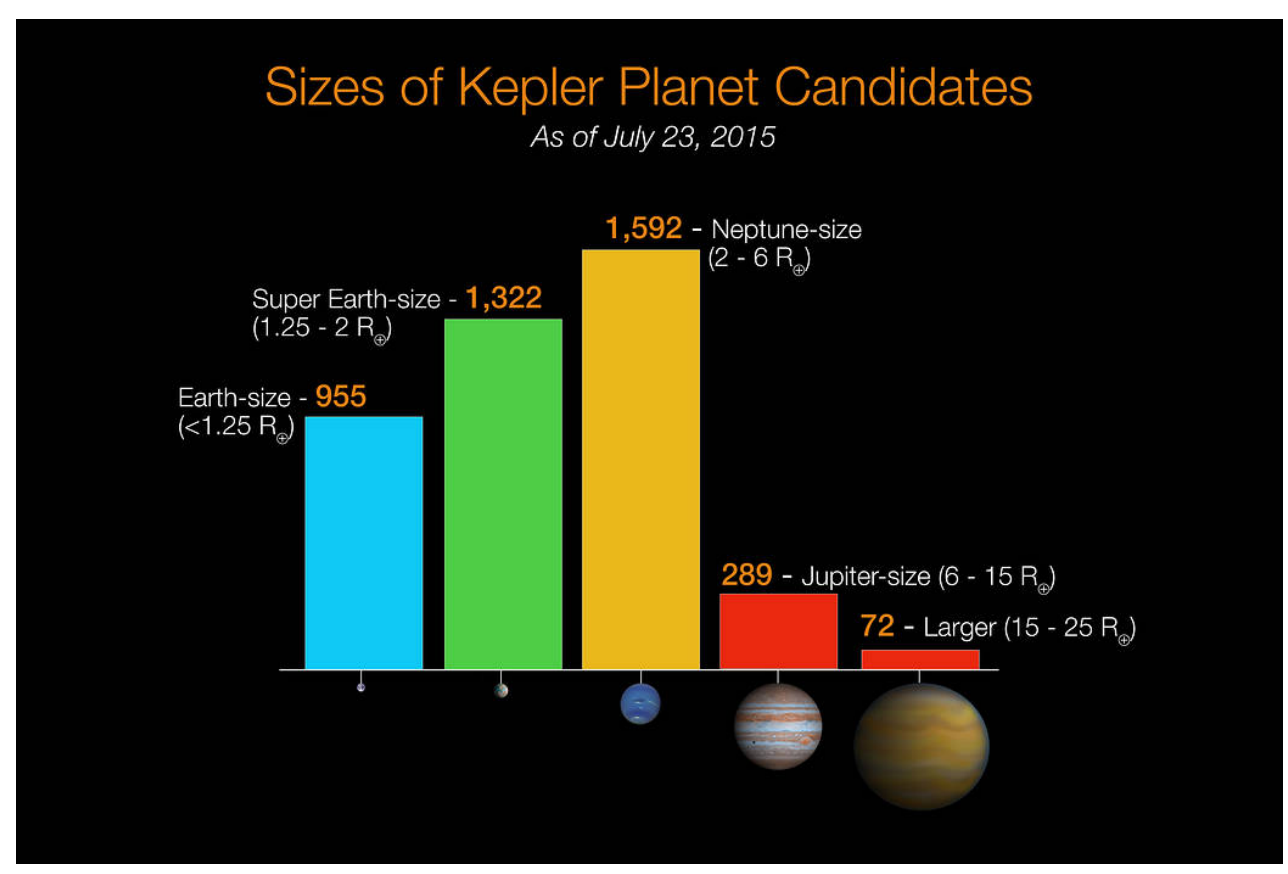

Figure 20: The distribution of Kepler planet candidates by size as of July 23, 2015 (Image Credit: NASA Ames/W Stenzel).

Figure 21 shows the radius versus orbital period of the discovered exoplanets as of December 14, 2017.

The research of potential habitable exoplanets has been strongly supported during last two decades. Indeed, this field of astrophysics is now probably the most exciting since the discovery of planets Earth-like could open a serious debate about the possibility of life outside of solar system. 


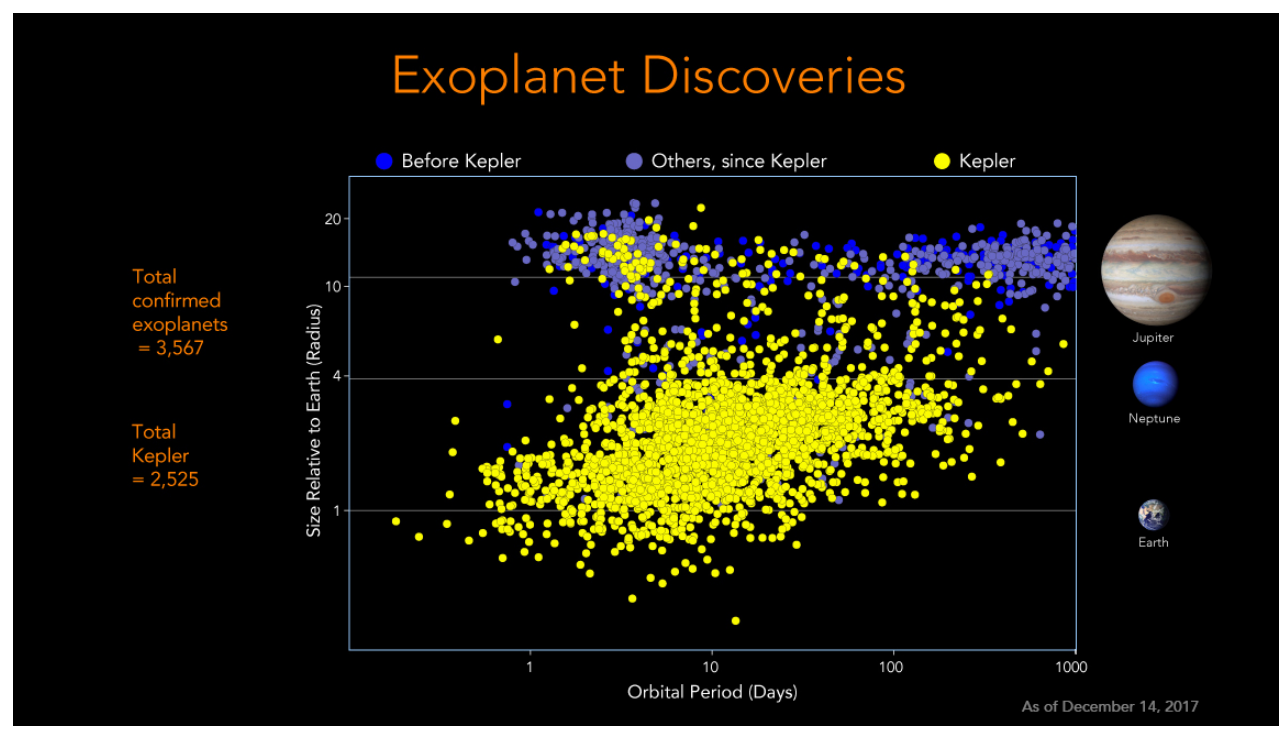

Figure 21: The radius versus orbital period of the discovered exoplanets as of December 14, 2017 (Image Credit: NASA [Public domain], via Wikimedia Commons).

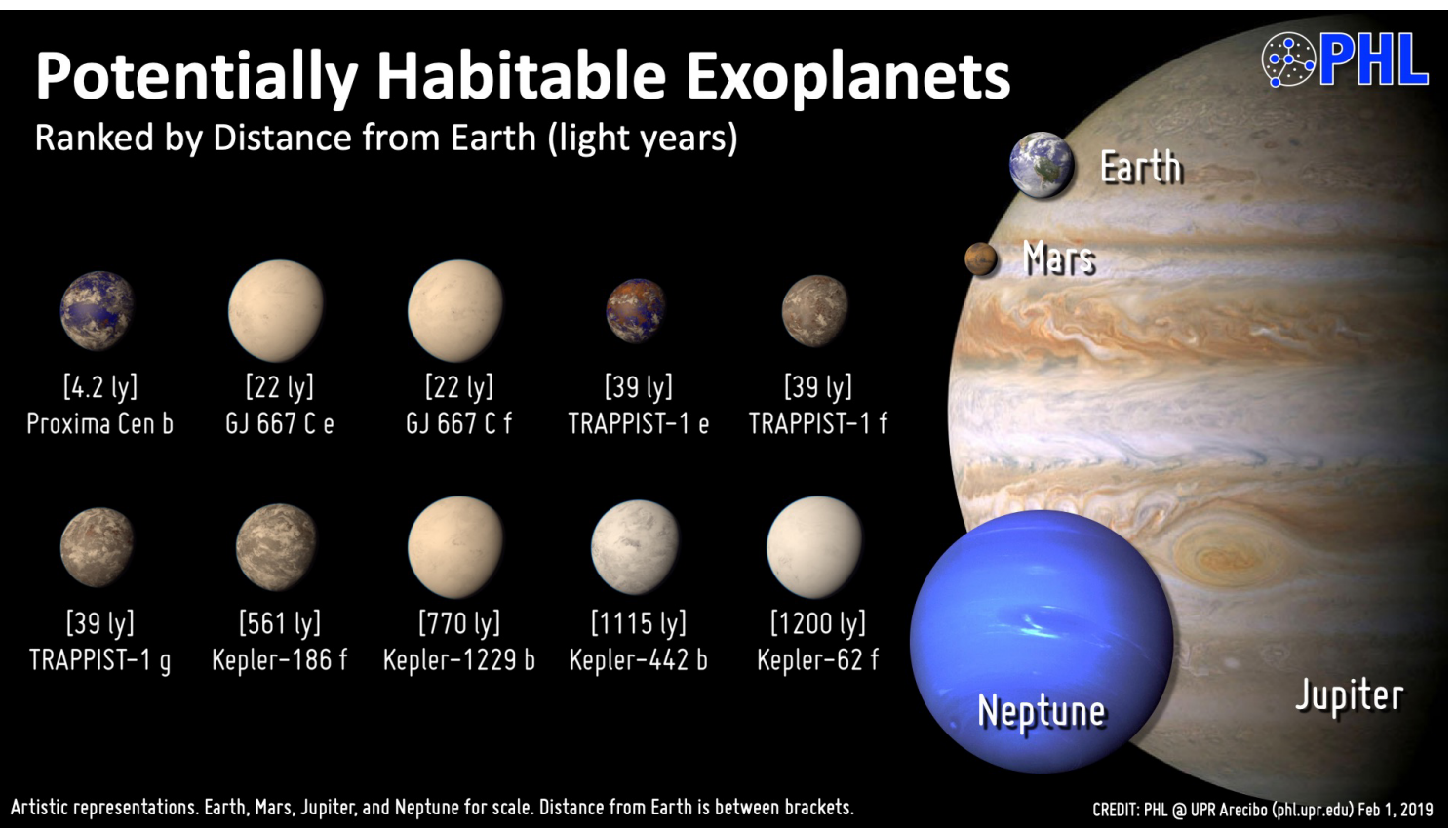

Figure 22: Current potential habitable exoplanets (2019, Credit: PHL@UPR Arecibo). 
The list of the potential habitable exoplanets updated to 1st February 2019 (Planetary Habitable Laboratory - PHL - University of Puerto Rico at Arecibo, http://phl.upr.edu/projects/habitableexoplanets-catalog) contains 49 objects: 16 Earth-size planets, 32 super-Earth-size planets, and 1 sub-Earth-size planet (Mars size). Figure 22 artistically shows such potential habitable exoplanets. This represents a list of the exoplanets that are more likely to have a rocky composition and maintain surface liquid water (i.e. $0.5<$ Planet Radius $\leq 1.5$ Earth radii or $0.1<$ Planet Minimum Mass $\leq 5$ Earth masses,and the planet is orbiting within the conservative habitable zone) (Credit: PHL@UPR Arecibo).

This list is continuously updated and the number of such exoplanets is rapidly increasing.

The Exoplanet Data Explorer is an interactive table and plotter for exploring and displaying data from the Exoplanet Orbit Database. The Exoplanet Orbit Database is a carefully constructed compilation of quality, spectroscopic orbital parameters of exoplanets orbiting normal stars from the peer-reviewed literature, and updates the Catalog of nearby exoplanets. A detailed description of the Exoplanet Orbit Database and Explorers was published by Han et al. (2014). The latest list in CSV format was updated on 2019/02/01 and is available at http://phl.upr.edu/hec.

The presence of numerous exoplanets in the vicinity of solar system - within a distance of $\sim$ $0.9 \mathrm{kpc}$, which is the range accessible by the Kepler mission - plays an important role in speculating about the possible number of such exoplanets within the whole habitable zone of our galaxy. Such habitable zone has an internal radius of $\sim 4 \mathrm{kpc}$ and an external radius of $\sim 11 \mathrm{kpc}$, as shown in Fig. 23 (after Lineweaver, Fenner \& Gibson, 2004), where the habitable zone in a Milky Way-like galaxy is represented in green. The number of stars contained in this zone is $\approx 10 \%$ of the total number of stars in the Galaxy. Taking into account that the thickness of the disk is $\approx 1 \mathrm{kpc}$, as evaluated by the differential rotation of the Galaxy, the habitable volume is $\sim 330 \mathrm{kpc}^{3}$. Therefore, if in a volume of $\approx 3.36 \mathrm{kpc}^{3}$ there are 955 Earth-size planets detected, in the habitable zone of our Galaxy we could expect $\approx 9.4 \times 10^{4}$ Earth-size planets, as lower limit. Planets around other stars are the rule rather than the exception, and there are likely hundreds of billions of exoplanets in the Milky Way alone (Maruyama, Ebisuzaki \& Kurokawa, 2018). Therefore, it is evident that the probability of finding numerous habitable planets becomes very high. Next generation instruments ground- and space-based will provide valuable information about this intriguing problem.

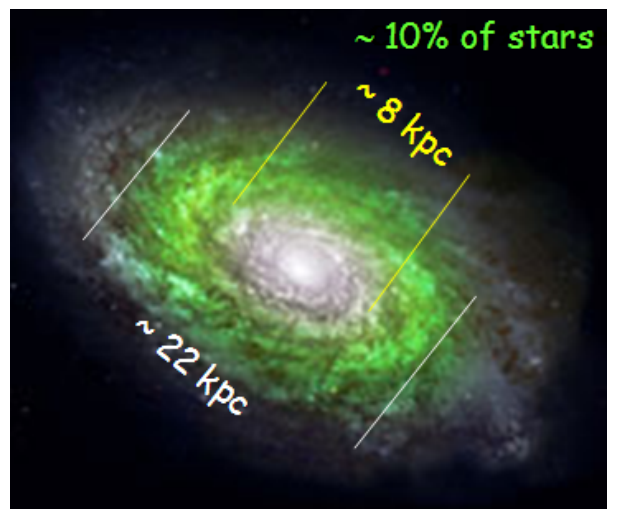

Figure 23: Habitable zone of a Milky Way-like galaxy (Giovannelli \& Sabau-Graziati, 2016 after Lineweaver, Fenner \& Gibson, 2004; Image courtesy: Yeshe Fenner, Space Telescope Institute). 
Studies about exoplanet predictions around stars have been performed by Bovaird \& Lineweaver (2013). They predict the existence of a low-radius $\left(R<2.5 R_{\odot}\right)$ exoplanet within the habitable zone of KOI-812 and that the average number of planets in the habitable zone of a star is 1-2.

For life-forms like us, the most important feature of Earth is its habitability. Understanding habitability and using that knowledge to locate the nearest habitable planet may be crucial for our survival as a species. During the past decade, expectations that the universe could be filled with habitable planets have been bolstered by the increasingly large overlap between terrestrial environments known to harbor life and the variety of environments on newly detected rocky exoplanets. The inhabited and uninhabited regions on Earth tell us that temperature and the presence of water are the main constraints that can be used in a habitability classification scheme for rocky planets. Lineweaver \& Chopra (2012) compiled and reviewed the recent exoplanet detections suggesting that the fraction of stars with planets is $\sim 100 \%$, and that the fraction with rocky planets may be comparably large. They reviewed extensions to the circumstellar habitable zone (HZ), including an abiogenesis habitable zone and the galactic habitable zone.

Earth is located in a dangerous part of the universe. Threats to life on Earth are manifold and range from asteroid impacts to supernova explosions and from supervolcano eruptions to humaninduced disasters. If the survival of the human species is to be ensured for the long term, then life on Earth has to spread to other planetary bodies. Mars is the most Earth-like planet we currently know and is the second closest planet; further it possesses a moderate surface gravity, an atmosphere, abundant water and carbon dioxide, together with a range of essential minerals. Thus, Mars is ideally suited to be a first colonization target. Here we argue that the most practical way that this can be accomplished is via a series of initial one-way human missions (Schulze-Makuch \& Davies, 2013).

However, we have interesting news about the presence of water in the universe. We knew that all the water found on Earth, has been transported by small bodies such as comets and asteroids. On the contrary, the work "The ancient heritage of water ice in the solar system" (Cleeves et al., 2014) has carried the knowledge one step further. It is understood that the water now present in Earth's oceans, and is present in other solar system bodies, has remained virtually unchanged with respect to that in the interstellar medium. This means that this water has not changed during the process of planet formation. This allows us to understand that the initial conditions that have favored the emergence of life are not unique, i.e. not dependent on the unique characteristics of our solar system. They can, however, be common in space.

Astrobiology is an interdisciplinary scientific field, recently born, not only focused on the search of extraterrestrial life, but also on deciphering the key environmental parameters that have enabled the emergence of life on Earth. Understanding these physical and chemical parameters is fundamental knowledge necessary not only for discovering life or signs of life on other planets, but also for understanding our own terrestrial environment. Recent papers by Cottin et al. (2017a,b) presented an interdisciplinary review of current research in astrobiology, covering the major advances and main outlooks in the field. They reviewed the most recent discoveries, the new understanding of planetary system formation including the specificity of the Earth among the diversity of planets, the origin of water on Earth and its unique combined properties among solvents for the emergence of life, the idea that the Earth could have been habitable during the Hadean Era, the inventory of endogenous and exogenous sources of organic matter and new concepts about how 
chemistry could evolve towards biological molecules and biological systems. In addition, many new findings show the remarkable potential life has for adaptation and survival in extreme environments. All those results from different fields of science are guiding our perspectives and strategies to look for life in other Solar System objects as well as beyond, in extrasolar worlds.

An intriguing question about the probability of finding a number of civilization in the Galaxy arises. It is now evident that Drake's formula (Drake, 1962) must be object of a robust revision.

For years, the search for manifestations of extraterrestrial civilizations has been one of humanity's most ambitious projects. Major efforts are now focused on the interception of messages from extraterrestrial civilizations, and the millimeter range is promising for these purposes (Dyson,1960). The Millimetron space observatory is aimed at conducting astronomical observations to probe a broad range of objects in the Universe in the wavelength range $20 \mu \mathrm{m}$ to $20 \mathrm{~mm}$, including the search for extraterrestrial life (Smirnov et al., 2012; Kardashev et al., 2014, and the references therein).

However, if we insist in looking for life which is like our own, why do we look for ... INTELLIGENT LIFE? (Giovannelli, 2001b).

Important news have been published by Anglada-Escudé et al. (2016). They reported observations that reveal the presence of a small planet with a minimum mass of about 1.3 Earth masses orbiting Proxima Centauri $(\mathrm{d}=1.295 \mathrm{pc})$ with a period of $\approx 11.2$ days at a semi-major-axis distance of $\sim 0.05 \mathrm{AU}$. Its equilibrium temperature is within the range where water could be liquid on its surface.

By using the European Southern Observatory's HARPS - a high precision instrument fitted to the 3.6-m telescope at the La Silla Observatory in Chile - 102 red dwarf stars neighbouring the sun over a period of six years have been studied. Red dwarfs are smaller and cooler than the Sun, however it's been found that $40 \%$ of red dwarf stars may have Earth-sized planets orbiting them that have the right conditions for life. New observations with Harps mean that about $40 \%$ of all red dwarf stars have a super-Earth orbiting in the habitable zone where liquid water can exist on the surface of the planet. Because red dwarfs are so common - there are about 160 billion of them in the Milky Way - this leads us to the astonishing result that there are tens of billions of these planets in our galaxy alone (Bonfils et al., 2013).

Therefore, planets around other stars are the rule rather than the exception, and there are likely hundreds of billions of exoplanets in the Milky Way alone. NASA's Kepler space telescope has found more than 2,400 alien worlds, including a new haul of 95 planets announced on Feb. 15, 2018.

A good help in finding and analyzing extrasolar planets can come from three recent simple programs:

- The TRAPPIST (TRAnsiting Planets and PlanetesImals Small Telescopes) consists in a network of two 60-cm robotic telescopes, one in Chile and one in Morocco, used by the Origins in Cosmology and Astrophysics (OrCA) researchers to contribute to the fields of astrobiology and planetology through two complementary approaches: the photometric detection and characterization of exoplanets transiting nearby stars (PI: Michael Gillon), and the photometric study of small bodies of our solar system (PI: Emmanuel Jehin) (Jehin et al., 2011). 
- The Search for habitable Planets EClipsing ULtra-cOOl Stars (SPECULOOS) Southern Observatory (SSO), a new facility of four 1-meter robotic telescopes at Cerro Paranal. The exquisite astronomical conditions at Cerro Paranal will enable SPECULOOS to detect exoplanets as small as Mars. It will explore approximately 1000 of the smallest $\left(\leq 0.15 \mathrm{R}_{\odot}\right)$, brightest $\left(\leq 12.5 \mathrm{~K}_{\mathrm{mag}}\right.$, and nearest $(\mathrm{d} \leq 40 \mathrm{pc}$ ) very low mass stars and brown dwarfs. The ultimate goals of the project are to reveal the frequency of temperate terrestrial planets around the lowest-mass stars and brown dwarfs, to probe the diversity of their bulk compositions, atmospheres and surface conditions, and to assess their potential habitability (Delrez et al., 2018; Jehin et al., 2018).

It is interesting to remark the importance of the book SPECULOOS Exoplanet Search and Its Prototype on TRAPPIST (Burdanov et al., 2018).

- ARIEL was conceived to observe a large number $(\sim 1000)$ of transiting planets for a chemical survey of exoplanets, including gas giants, Neptunes, super-Earths and Earth-size planets around a range of host star types using transit spectroscopy in the $1.25-7.8 \mu \mathrm{m}$ spectral range and multiple narrow-band photometry in the optical. ARIEL will thus provide a representative picture of the chemical nature of the exoplanets and relate this directly to the type and chemical environment of the host star (Tinetti et al., 2018).

Following the NASA Exoplanet Exploration page (https://exoplanets.nasa.gov/the-search-forlife/life-signs/) we can say that our early planet finding missions, such as NASA's Kepler and its new incarnation, K2, or the soon-to-be-launched James Webb Space Telescope, could yield bare bones evidence of the potentially habitable worlds. Perhaps K2's examination of nearer, brighter stars will stumble across an Earth-sized planet in its star's habitable zone, close enough for follow ups by other instruments to reveal oceans, blue skies and continents. Or James Webb, designed in part to investigate gas giants and super Earths, might find an outsized version of our planet. With a possible launch in the mid 2020s, WFIRST (Wide-Field Infrared Survey Telescope), could zero in on a distant planet's reflected light to detect the signatures of oxygen, water vapor, or some other powerful indication of possible life.

But unless we get lucky, the search for signs of life could take decades. Discovering another blue-white marble hidden in the star field, like a sand grain on the beach, will probably require an even larger imaging telescope. Designs are already underway for that next-generation planet finder, to be sent aloft in the 2030s or 2040s.

However, we are going to study 50,000 Clusters of Galaxies (Thomas Boller, 2017), and we have millions more. We know the lower limit of the extrapolated number of Earth-size planets in the habitable zone of our Galaxy. Thus a natural extrapolation can be reasonably thought. Thus, we can dare to say that we are approaching to the philosophical results obtained by two great free thinkers:

- Siddhartha Gautama also known as Shakyamuni (the sage of Shaka - between the VI and V century B.C.) who exposes a grandiose vision of the universe: through the concept of "major system of worlds", a concept on huge scale that implies both the existence of countless galaxies and the possibility of sentient life on other planets other than our own (from the Lotus Sutra - the central text of Mahayana Buddhism). 


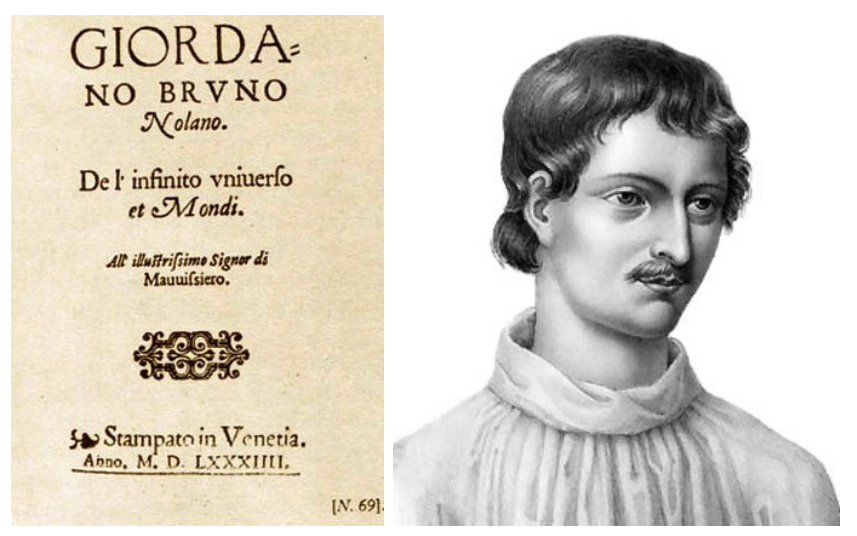

Figure 24: Left panel: Frontispiece of the original publication of De l'infinito, universo et mondi (Giordano Bruno, 1584). Right panel: Giordano Bruno (Rixner \& Siber, 1824).

- About 2000 years later, Giordano Bruno (Nola 1548 - Roma 17th February 1600) who was burned alive in Campo dei Fiori by the "Saint Inquisition" because of his thought - summarized in De l'infinito, universo e mondi (Giordano Bruno, 1584) (see Fig. 24) - that produced the same conclusions of Siddharta: The Universe is infinite and is populated by a myriad of worlds. Moreover he was saying that "Whether we like it or not, we are the cause of ourselves. Being born in this world, we fall into the illusion of the senses: we believe in what appears. We ignore that we are blind and deaf. Then the fear attacks us and we forget that we are "divine". We can change the course of events".

These philosophical lucubrations were not exactly in agreement with the position of the Roman (Catholic) Church!

Of course we must wait scientific confirmation for the "alien life". We must wait even more for the discovery of "intelligent life". But, the number of discovered planets is growing very fast. Thus, we can reasonably affirm that the Universe is full of life, hoping to avoid to be burned alive like Giordano Bruno.

It is important for me to close this section with the beautiful words full of hope of William Borucki - principal investigator for NASA's Kepler mission: If we find lots of planets like ours... we'll know it's likely that we aren't alone, and that someday we might be able to join other intelligent life in the universe.

\section{Accretion onto compact objects}

For a general deep tutorial lecture see "Black Hole Accretion and Feedback" by Andrew King (2018).

In the Galaxy there are different kinds of compact sources: white dwarfs (WDs), neutron stars (NSs) and black holes (BHs), both isolated and in binary systems. Thousand papers about these cosmic sources are available in the literature. We mention the last available exhaustive review by Postnov \& Yungelson (2014) about "The Evolution of Compact Binary Stars Systems in which they review the formation and evolution of compact binary stars consisting of WDs, NSs, and BHs. 
Merging of compact-star binaries are expected to be the most important sources for forthcoming GW astronomy.

Several review papers have been published for discussing the different classes of galactic compact sources: i) Cataclysmic Variables (CVs) and related objects (e.g. Giovannelli \& SabauGraziati, 2008, 2012b; 2015b); ii) High Mass X-ray Binaries (HMXBs) (e.g. Giovannelli \& SabauGraziati, 2001, 2004, 2014b, and van den Heuvel, 2009 and references therein; iii) Obscured Sources and Supergiant Fast X-Ray Transients (e.g. Chaty, 2011); iv) Ultra-Compact DoubleDegenerated Binaries (e.g. Wu, Ramsay \& Willes, 2008; Wu, 2009); Magnetars (Kitamoto et al., 2014: White Paper for ASTRO-H Space X-ray observatory).

A summary of these topics can be found in the review paper by Giovannelli \& Sabau-Graziati (2014a).

Among the cosmic systems where accretion processes occur, undoubtedly, non-magnetic CVs, intermediate polars and polars constitute the most powerful probe to test our theories of the various modes of accretion. The reason is rather simple: $\mathrm{CVs}$ are enough close to us and their processes develop in time-scales relatively easy to be followed and enough energetic to be easily detected.

The accretion structure depends on the magnetic field (B) of the white dwarf and on the transfer mass rate.

Figure 25 (Bisikalo \& Zhilkin, 2015) shows from the left to the right panels the 3D MHD simulations of the so-called non-magnetic CVs (NMCVs) $\left(\mathrm{B} \sim 10^{4}-10^{5} \mathrm{G}\right)$, intermediate polars (IPs) $\left(\mathrm{B} \sim 10^{6}-10^{7} \mathrm{G}\right)$, and polars (Ps) $\left(\mathrm{B} \sim 10^{7}-10^{8} \mathrm{G}\right)$.
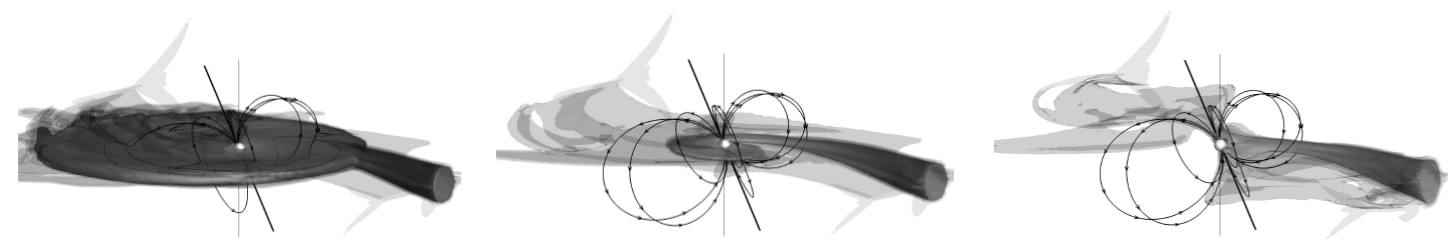

Figure 25: Left panel: non-magnetic CV. Central panel: intermediate polar. Right panel: polar. The 3D MHD simulations were obtained with $\mathrm{B}=10^{5}, 10^{6}$, and $10^{7} \mathrm{G}$, respectively (Bisikalo \& Zhilkin, 2015).

However we have a smooth continuity among the classes, as shown in fig. 26 (Giovannelli \& Sabau-Graziati, 2015a).

Indeed, taking into account the average values of magnetic field intensity and orbital periods for polars and IPs, and the minimum and maximum value for both parameters ( $\mathrm{B}$ and $\mathrm{P}_{\mathrm{orb}}$ ), it is possible to construct a very interesting plot (Fig. 26) that shows the evident continuity between the two classes of MCVs. Such a continuity has been noted by Schmidtobreick \& Tappert $(2014,2015)$ : CVs evolution is driven by angular momentum loss; as consequence $\mathrm{P}_{\text {orb }}$ decreases. All long $\mathrm{P}_{\text {orb }}$ CVs cross SW Sex regime before entering in the "period gap". Therefore SW Sex phenomenon is an evolutionary stage in the life of CVs (e.g. Rodriguez-Gil, 2003).

The long term evolution of CV systems accreting at a prohibitive rate has become a hot topic both in terms of the fate of such systems (all sorts of supernovae) and the microphysics of Eddington and super Eddington mass accretion and mass loss flows. In particular we stress one of the hottest topics in present day astrophysics, namely the progenitors of SN-Ia. This problem is connected with fundamental issues in cosmology. Novae and recurrent novae are the most promising 


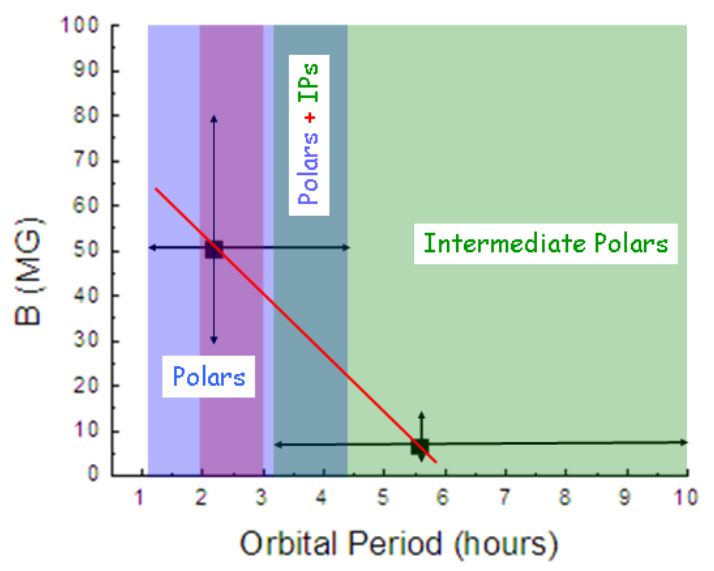

Figure 26: Magnetic field intensity versus orbital period for MCVs. Polars and IPs are contained in the light blue and light green rectangles, respectively. Violet rectangle indicates the so-called "period gap". Cyan-50 rectangle represents the intersection between the Polars and IPs (adopted from Giovannelli \& Sabau-Graziati, 2015a).

progenitor candidates but so far could not be nailed down. One of the most important goals of our serial workshops about The Golden Age of Cataclysmic Variables and Related Objects is to discuss what is missing in our knowledge of CVs that will allow a unique determination and observational confirmation (Giovannelli \& Sabau-Graziati, 2012a, 2015b,c, 2017). Indeed the sub-class of CVs, named Classical Novae $(\mathrm{CNe})$, which are the third most powerful stellar explosions in a galaxy, have been observed as close as a kpc and as far as galaxies in the Fornax cluster.

The recent renaissance in $\mathrm{CNe}$ studies is coming from the observations with 8-10m class telescopes, high resolution spectroscopy, in synergy with observations from space carried out with Swift, XMM, Chandra, HST, and Spitzer. This, coupled with recent advances in the theory of the outburst, can provide important advancement in the knowledge of the physics governing such a powerful accretors.

Since long time it appeared evident that the old classification of CVs based on the optical outburst properties, by which one may distinguish four groups:

- classical novae;

- recurrent novae;

- dwarf novae;

- nova-like objects

is neither self-consistent nor adequate and it is much better to consider primarily the observed accretion behaviour (Smak, 1985a,b). Therefore, it is necessary to find a method as general as possible to describe the behavior of cataclysmic variables.

The nature in all its manifestations shows continuity. Then we have to abandon the "convenient method" of thinking everything in Watertight Compartments and to go toward a general model for 
compact accreting stars. This can be obtained looking at the "Accretion Behaviour" and "Magnetic Fields".

This was the fundamental concept of The Scenario Machine, an idea developed by Vladimir Lipunov and collaborators many years ago.

\subsection{The Scenario Machine}

Starting from the trivial definition of X-ray Binary Systems (XRBs): they are binary systems emitting X-rays, a natural question arises. Are these systems governed by few physical parameters independent of their nature? The answer is positive. Indeed, High Mass XRBs (HMXRBs), Low Mass XRBs (LMXRBs), Anomalous X-ray Pulsars (AXPs), and Cataclysmic Variables (CVs) can be considered as gravimagnetic rotators: a body with mass $\mathrm{M}$, having a magnetic moment $\vec{\mu}$, rotating with rotational velocity $\vec{\omega}$, being the two axis not necessarily coincident, as sketched in Fig. 27 (Giovannelli, 2016). Introducing a physical parameter, $y=\dot{M} / \mu^{2}$, named gravimagnetic parameter, all the gravimagnetic rotators are contained in a plane $\log \mathrm{P}_{\text {spin }}$ vs $\log \mathrm{y}$ (Lipunov, 1987; Lipunov \& Postnov, 1988).

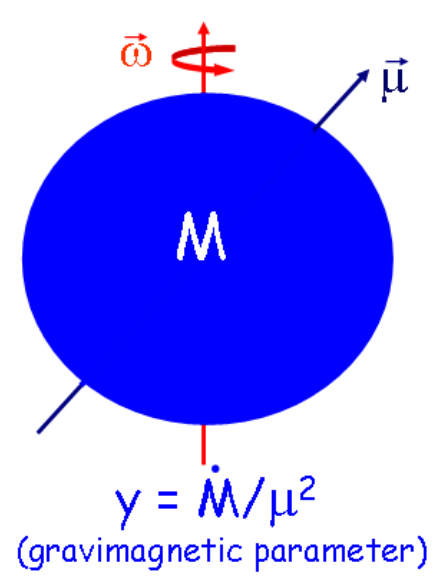

Figure 27: Gravimagnetic rotator: a body with mass $M$, having a magnetic moment $\vec{\mu}$, rotating with rotational velocity $\vec{\omega}$ (Giovannelli, 2016). The parameter $y=\dot{M} / \mu^{2}$ is called gravimagnetic parameter (Lipunov, 1987; Lipunov \& Postnov, 1988).

The Scenario Machine (Monte Carlo simulations of binary evolution) permits to build up the complete picture of all possible evolutionary stages of binaries in the Galaxy. The basic evolution equation (5.1) used for 500,000 systems containing magnetized stars provided the results contained in the plane $\log \mathrm{P}_{\text {spin }}-\log \mathrm{y}$, reported in the upper panel of Fig. 28. $\mathrm{P}_{\text {spin }}$ is expressed in seconds and the gravimagnetic parameter is expressed in unit of $10^{-42} \mathrm{~g} \mathrm{~s}^{-1} \mathrm{G}^{-2} \mathrm{~cm}^{-6}$. The symbols used for the different types of binaries are explained in the lower panel of Fig. 28. The definition of the characteristic radii can be found in the paper by Lipunov (1987). Observational examples of various types of rotators are reported in Fig. 29 (Lipunov, 1987).

$$
\frac{\mathrm{dI} \omega}{\mathrm{dt}}=\dot{\mathrm{M}} \mathrm{K}_{\mathrm{su}}-\frac{\kappa_{\mathrm{t}} \mu^{2}}{\mathrm{R}_{\mathrm{t}}^{3}}
$$




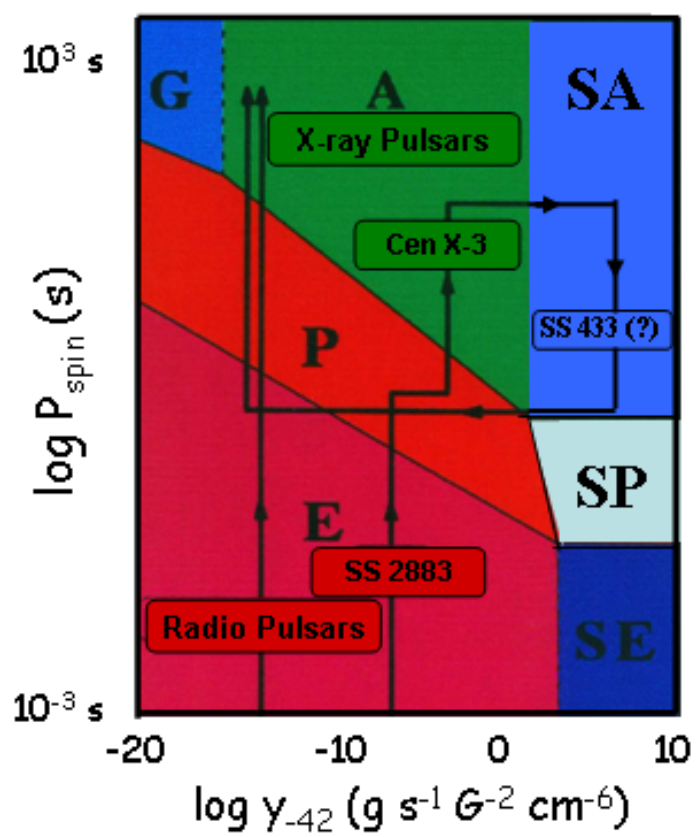

The rotator classification

\begin{tabular}{|c|c|c|}
\hline Designation & Name & Physical sense \\
\hline $\mathrm{E}$ & Ejector & $R_{s t}>\max \left\{R_{G}, R_{l}\right\}$ \\
\hline $\mathbf{P}$ & Propeller & $R_{c}<R_{s t} \leq \max \left\{R_{G}, R_{l}\right\}$ \\
\hline A & Accretor & $R_{s t} \leq R_{G}$ and $R_{s t} \leq R_{c} \quad \dot{M}_{c} \leq \dot{M}_{c r}$ \\
\hline G & Georotator & $R_{G}<R_{s t} \leq R_{c}$ \\
\hline M & Magnetor & $R_{s t}>a$ and $R_{c}>a$ \\
\hline SE & Superejector & $R_{s t}>R_{l}$ \\
\hline SP & Superpropeller & $R_{c}<R_{s t} \leq R_{l}$ \\
\hline SA & Superaccretor & $R_{s t} \leq R_{c}$ and $R_{s t} \leq R_{G}$ \\
\hline
\end{tabular}

Figure 28: Upper panel: distribution of magnetic rotators in the plane "Spin Period" - "Gravimagnetic Parameter" (adapted from Lipunov, 1995); lower panel: classification of rotators (Lipunov, 1987).

\begin{tabular}{|c|c|c|c|}
\hline Type of rotator & Designation & $\begin{array}{l}\text { The clearly confirmed } \\
\text { observational example }\end{array}$ & Model assumptions \\
\hline Ejector & $\mathrm{E}$ & Radiopulsars & $\begin{array}{l}\text { LSI }+61^{\circ} 303, \text { Cyg X-3, } \\
\text { BL Lac objects, }\end{array}$ \\
\hline Propeller & $P$ & - & $\begin{array}{l}\text { Transient X-ray sources, } \\
\gamma \text {-bursts, some cataclysmic } \\
\text { variables (dwarf novae), } \\
\text { magnetic Ap-stars }\end{array}$ \\
\hline Accretor & A & $\begin{array}{l}\text { X-ray pulsars, X-ray } \\
\text { bursters, cataclysmic } \\
\text { variables with white } \\
\text { dwarfs, novae, intermediate } \\
\text { polars }\end{array}$ & - \\
\hline Superejector & SE & - & SS 433, AGN, QSO \\
\hline Superpropeller & SP & - & - \\
\hline Superaccretor & SA & - & SS 433 \\
\hline Georotator & $\mathrm{G}$ & - & - \\
\hline Magnetor & M & Polars & - \\
\hline
\end{tabular}

Figure 29: Observational examples of rotators (Lipunov, 1987). 
where:

$\mathrm{K}_{\mathrm{su}}=$ specific angular momentum applied by the accretion matter to the rotator;

$\mathrm{K}_{\mathrm{su}}=\sqrt{\mathrm{GM}_{\mathrm{x}} \mathrm{R}_{\mathrm{d}}}$ for Keplerian disk accretion;

$\mathrm{K}_{\mathrm{su}}=\eta_{\mathrm{t}} \Omega R_{\mathrm{g}}^{2}$ for wind accretion in a binary;

$\mathrm{K}_{\mathrm{su}} \sim 0$ for a single magnetic rotator;

$R_{d}=$ radius of the inner disk edge;

$\Omega=$ rotational frequency of the binary system;

$\eta_{\mathrm{t}}=1 / 4 \quad$ (Illarionov \& Sunyaev, 1975);

$\kappa_{\mathrm{t}}=$ dimensionless factor;

$\mathrm{R}_{\mathrm{t}}=$ characteristic radius;

$\dot{M}=$ accretion rate in different regimes.

Using the "Scenario Machine" Raguzova \& Lipunov (1999) obtained an evolutionary track that can lead to the formation of $\mathrm{Be} / \mathrm{BH}$ systems. The modern evolutionary scenario predicts the existence of binary black holes on eccentric orbits around Be stars and such systems may be discovered in the near future... Like happened! Indeed MWC 656, the first Be/BH binary was detected by Casares et al. (2014).

Raguzova \& Lipunov (1999) calculations show that binary black holes with Be stars must have $0.2<\mathrm{e}<0.8$. It is particularly difficult to detect such systems as most of their spectroscopic variations occur in a relatively small portion of the orbit, and could easily be missed if the systems are observed at widely separated epochs. They found for 20-30 Be/NS systems the formation of 1 $\mathrm{Be} / \mathrm{BH}$ system.

Belczynski \& Ziółkowski (2009) used binary population synthesis models to show that the expected ratio of $\mathrm{Be} / \mathrm{XRBs}$ with neutron stars to black holes in the Galaxy is relatively high: for $~$ $30-50 \mathrm{Be} / \mathrm{NS}$ systems the formation of $1 \mathrm{Be} / \mathrm{BH}$ system, and so broadly in line with observations.

\subsection{Accretion driven X-ray sources}
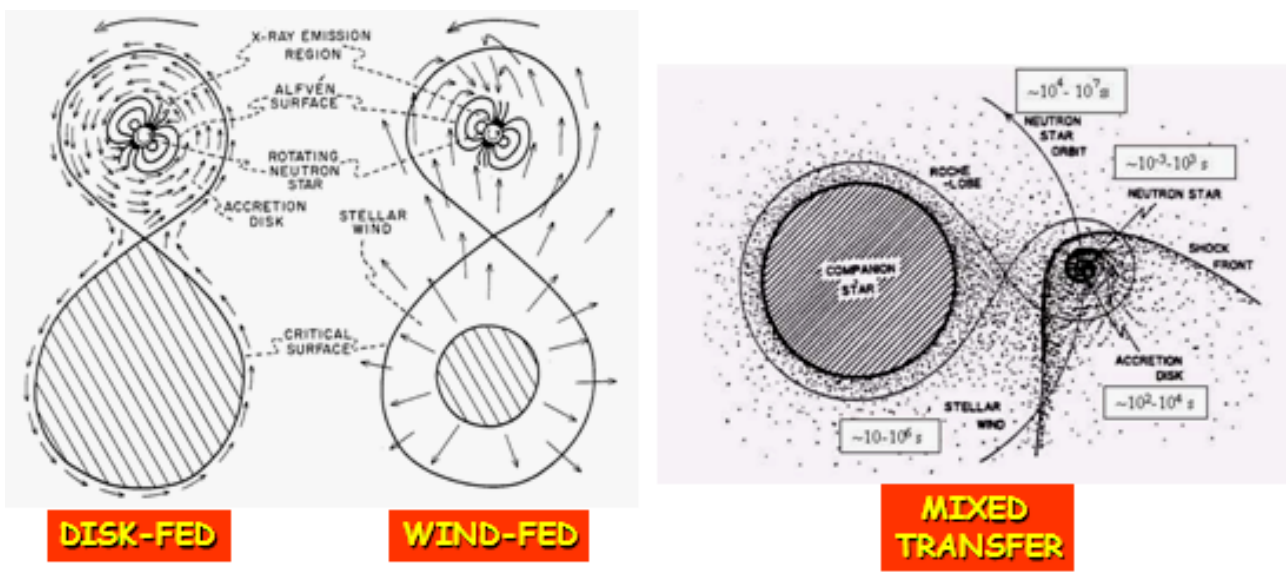

Figure 30: Left panel: accretion in X-ray binary systems disk-fed and wind-fed (Giovannelli \& SabauGraziati, 2001, adapted from Blumenthal \& Tucker, 1974). Right panel: mixed transfer (adopted from Giovannelli \& Sabau-Graziati, 2001, after Nagase, 1989). 
In binary systems there are essentially two ways for accreting matter from one star to the other: via accretion disk or via stellar wind (Giovannelli \& Sabau-Graziati, 2001, adapted from Blumenthal \& Tucker, 1974) (left panel of Fig 30). But in some cases there is a third way which is a mixture between the two, as for instance in eccentric binary systems close to the periastron passage where a temporary accretion disk can be formed around the neutron star (e.g. Giovannelli \& Ziółkowski (1990), like shown in the right panel of Fig. 30 (Giovannelli \& Sabau-Graziati, 2001, after Nagase, 1989).

\subsection{Doppler tomography of accretion disks}

The fast Fourier Transform (FFT) in one, two, or three dimensions is an efficient algorithm well known for decades (see Cooley \& Tukey, 1965) as well as a traditional component of any serious numerical library. Three-dimensional (3D) FFT happens to be one of the most compute- and communication-intensive components in applications from a range of fields (for example, in turbulence, molecular dynamics, 3D tomography, and astrophysics), and for this reason there are many excellent parallel implementations in existence today. Pekurovsky (2012) introduced a popular software package called P3DFFT which implements FFT in three dimensions in a highly efficient and scalable way. This paper discussed P3DFFT implementation and performance in a way that helps guide the user in making optimal choices for parameters of their runs.

Horne (1985) and Marsh \& Horne (1988) developed Doppler tomography as a technique aimed at constructing a two-dimensional velocity image (tomogram) of the accretion disks of CVs using an emission line in its spectra sampled at a number of orbital phases. Doppler tomography has revolutionized the interpretation of orbitally phase-resolved spectroscopic observations of interacting binary systems. It has become a valuable tool for resolving the distribution of line emission in CVs and other binary systems. For instance Marsh (2001) in his review discussed the results of Doppler tomography applied to CVs. Outstanding successes to that date are the discovery of two-arm spiral shocks in cataclysmic variable accretion disks and the probing of the stream/magnetospheric interaction in magnetic cataclysmic variable stars. Doppler tomography has also told us much about the stream/disk interaction in non-magnetic systems and the irradiation of the secondary star in all systems.

Zhilkin \& Bisikalo (2009) described a 3D numerical code designed for simulations of magnetohydrodynamical (MHD) flows in semidetached binary systems. They found that even in case of a moderate magnetic field $\left(\mathrm{B}=10^{5} \mathrm{G}\right)$ the flow structure in the vicinity of the accretor of a CV was drastically changed and the funnel flows were formed in the system.

Bisikalo \& Zhilkin (2012) discussed the Flow Structure in Magnetic CVs by studying the physical processes which go on in magnetic CVs with the mass transfer between the components. Using results of 3D MHD simulations, they investigated variations of the main characteristics of accretion disks depending on the value of the magnetic induction on the surface of the accreting star. Zhilkin, Bisikalo \& Mason (2012) discussed on "Full 3D MHD calculations of accretion flow structure in magnetic cataclysmic variables with strong, complex magnetic fields".

Bisikalo et al. (2008) discussed Dopppler tomography of the CV SS Cygni in quiescence. Boneva et al. (2009) discussed the Doppler mapping in the case of SS Cygni in outburst and finally Kononov et al. (2012) used Doppler tomography for studying the pre-outburst accretion disk of SS Cygni. 
Zhilkin \& Bisikalo $(2010,2011)$ presented a 3D numerical model to simulate plasma flows in close binary systems with accretors having strong proper magnetic fields. The model is based on an assumption that plasma dynamics is determined by a slow average flow against a background of propagating MHD waves. Equations describing the slow plasma motion are obtained as a result of the corresponding averaging procedure. The model takes into account the magnetic diffusivity which is due to the current dissipation in turbulent vortexes, magnetic buoyancy and wave MHD turbulence. They presented results of the simulations for MHD flows in a polar-like binary system. Results of the simulations show that in semi-detached binary systems with strong magnetic fields $\left(B=10^{7} \mathrm{G}\right)$ the flow structure can differ sufficiently from that of systems with moderate magnetic fields $\left(B=10^{5} \mathrm{G}\right)$, Accretion disks in such magnetic systems systems $\left(B=10^{7} \mathrm{G}\right)$ are not formed and the flow is collimated into the accretion stream starting from the inner Lagrange point $\mathrm{L}_{1}$ and finishing on the magnetic poles of the accretor. This flow structure corresponds to the case of polars.

Kotze, Potter \& McBride $(2015,2016)$ explored inside-out Doppler tomography for nonmagnetic and magnetic cataclysmic variables, respectively. The code they used is available at http://www.saao.ac.za/ ejk/doptomog/main.html.

Doppler tomography has been applied also to fusion plasmas giving an image of the fastion velocity distribution function in the tokamak ASDEX Upgrade (Salewski et al., 2015). It is interesting to note that Bisikalo et al. (2016) presented a method that can be used to recover the spectrum of turbulence from observations of optically thin emission lines formed in astrophysical disks. Within this method, they analyze how line intensity fluctuations depend on the angular resolution of the instrument, used for the observations. The method allows us to restore the slope of the power spectrum of velocity turbulent pulsations and estimate the upper boundary of the turbulence scale.

Doppler tomography is very useful also for analyzing the asymmetric MHD outflows/jets from accreting T Tauri stars, and even in planetary science, as discussed by Giovannelli \& Sabau-Graziati (2016, and the references therein).

\subsection{Accretion onto white dwarfs}

Accretion of matter onto WD can take place through a disk, a ring-like structure or an accretion stream depending on the WD magnetic field strength, the binary orbital period and mass accretion rate. The physical conditions of the material impinging onto the WD and emitting X-rays are still to be investigated. In non-magnetic CVs the WD magnetic field strength is low enough, $\mathrm{B}_{\mathrm{WD}} \lesssim 10^{5}$ $\mathrm{G}$, to allow the formation of an accretion disk and $\mathrm{X}$-rays are emitted at the disk Boundary Layer (BL). In highly magnetized CVs, $\mathrm{B}_{\mathrm{WD}} \gtrsim 10^{7} \mathrm{G}$, material is directly accreted onto the WD poles flowing along the magnetic field lines. A partial accretion disk (a sort of ring) can be formed at intermediate field strengths $\left(10^{5} \lesssim \mathrm{B}_{\mathrm{WD}} \lesssim 10^{7} \mathrm{G}\right.$ (see Fig. 25 , where the 3D MHD simulations of the three cases are reported).

At the WD poles a stand-off shock is formed. The post-shock accretion column is thought to cool via bremstrahlung (hard X-rays), recombination processes and cyclotron radiation. The relative proportion of these cooling mechanisms strongly depends on the WD magnetic field (Fischer \& Beuermann 2001). An interesting discussion about Dissecting accretion and outflows in accreting white dwarf binaries is reported by de Martino et al. (2015). 
Therefore, the magnetic field intensity plays a fundamental role in the process of accretion of matter onto the compact star. Thus Lipunov's diagram $\left(\log \mathrm{P}_{\text {spin }}\right.$ vs $\left.\log \mathrm{y}\right)$ appears as the best way for localizing the position of MWDs, both polars and IPs, as shown in Fig. 31. In this figure, also the positions of other gravimagnetic rotators are shown.

Sion (http://astronomy.villanova.edu/faculty/sion/CV/index.html) states that in the Galaxy we could expect $\approx 10^{6} \mathrm{CVs}$. One of the big questions that arises is: "can all of the observed CVs and the phenomena associated with them be understood in terms of a single unified picture?" Other questions relate to the relative probabilities that CVs will be observed at particular stages in their evolution, and how the observations of CVs at the current epoch can be used to determine their ultimate fate. To address these questions Nelson (2012) and Goliasch \& Nelson (2015) have undertaken a massive computational effort to theoretically simulate the evolution of most of the possible CVs that could be produced by nature. The temporal evolution of 56,000 nascent CVs was followed over an age of 10 billion years using the MESA stellar evolution code. According to Nelson, "This is the most ambitious analysis of the properties of an entire CV population that has ever been

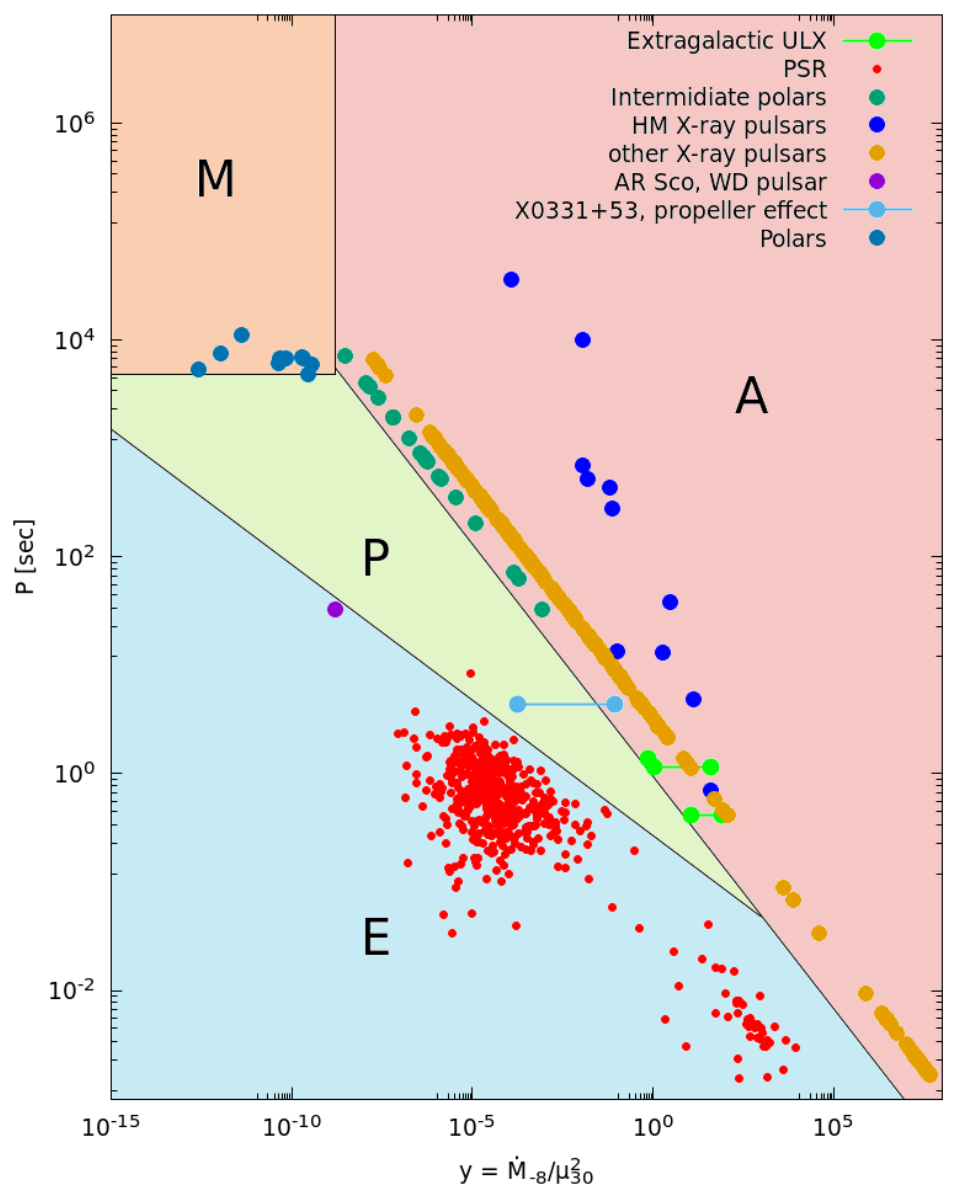

Figure 31: Lipunov's diagram: the position of different kind of gravimagnetic rotators are shown as well as those of several polars and IPs (courtesy of Vladimir Lipunov, 2018). 
undertaken. The whole project required several core-years of CPU time."

While many of the results confirmed what had already been inferred about the properties of $\mathrm{CVs}$, there were a number of surprises including the identification of a number of previously unexplored evolutionary pathways. But, as expected, a sharp bifurcation was found between nascent $\mathrm{CVs}$ that evolved to produce double white-dwarf binaries (including ones containing helium and hybrid white dwarfs), and ones that continuously transferred mass over the lifetime of the universe. In addition, the predictions of the theoretical simulations were in good general agreement with the observations of CVs with reasonably well-measured properties.

What was surprising was the large number of short-period "ultracompact" binaries (AM CVn stars) that were produced and, especially, the enormous depletion of carbon relative to nitrogen and oxygen that is predicted at certain epochs for evolved systems. As Nelson points out, "It seems that nature has provided us with a unique way to identify CVs that descended from a highly evolved state based on their carbon abundances. There is already some observational evidence to suggest that there is a significant depletion of carbon in certain CVs. This could be a really critical test that will allow us to infer the lineage of some CVs and predict what their fate will be".

\subsection{Accretion onto Neutron Stars and Black Holes}

In LMXBs (Low-Mass X-ray Binaries) the compact object can be either a neutron star or a black hole and the optical companion is a low mass star. The exchange of matter occurs via Roche lobe overflow, like shown in the left panel of Fig. 30. In HMXBs (High-Mass X-ray Binaries) the compact object can be either a neutron star or a black hole and the optical companion is a high mass star: giant or super-giant. The exchange of matter occurs mainly via stellar wind since usually the optical star does not fill its Roche lobe (Fig. 30, left panel). However, sometimes, the exchange of matter can occur in a mixed way because of the formation of an accretion disk around the compact object around the periatron passage (e.g. Giovannelli \& Ziółkowski, 1990) (Fig. 30, right panel).

McClintock, Narayan \& Rybicki (2004) found a very interesting relationship between the minimum X-ray luminosity in the range $0.5-10 \mathrm{keV}$ and the orbital periods of BH LMXBs and NS LMXBs. BH LMXBs are on average a factor of $\sim 100$ fainter than NS LMXBs with similar orbital periods (Fig. 32).

As well known, X-ray/Be systems are formed by a compact star and an optical star. Obviously there is a mutual influence between the two stars. Low-energy (LE) processes influence highenergy (HE) processes and vice versa. Never confuse the effect with the cause. There is a general law in the Universe: Cause and Effect. The Cause generates an Effect and NOT vice versa!

Time-lag between HE events and LE events in disk-fed accreting X-ray binaries (XRBs) has been noted in many systems, but the trigger of the work resulted in a model for explaining in general such a phenomenon (Bisnovatyi-Kogan \& Giovannelli, 2017) was given by Giovannelli \& SabauGraziati (2011) who noted a systematic delay between the relative enhancement in luminosity of the optical Be star - occurring at the periastron passage of the neutron star - and the subsequent X-ray flare in the system HDE 245770/A 0535+26. The model for such a system was developed and corroborated by many events (Giovannelli, Bisnovatyi-Kogan \& Klepnev, 2013: GBK13), and later by events reported in Giovannelli et al. (2015) where also a relationship between $\Delta \mathrm{V}_{\text {mag }}$ of the optical star at the periastron and X-ray intensity ( $\left.\mathrm{I}_{\mathrm{X}}\right)$ of the 8-day delayed flare was produced. 


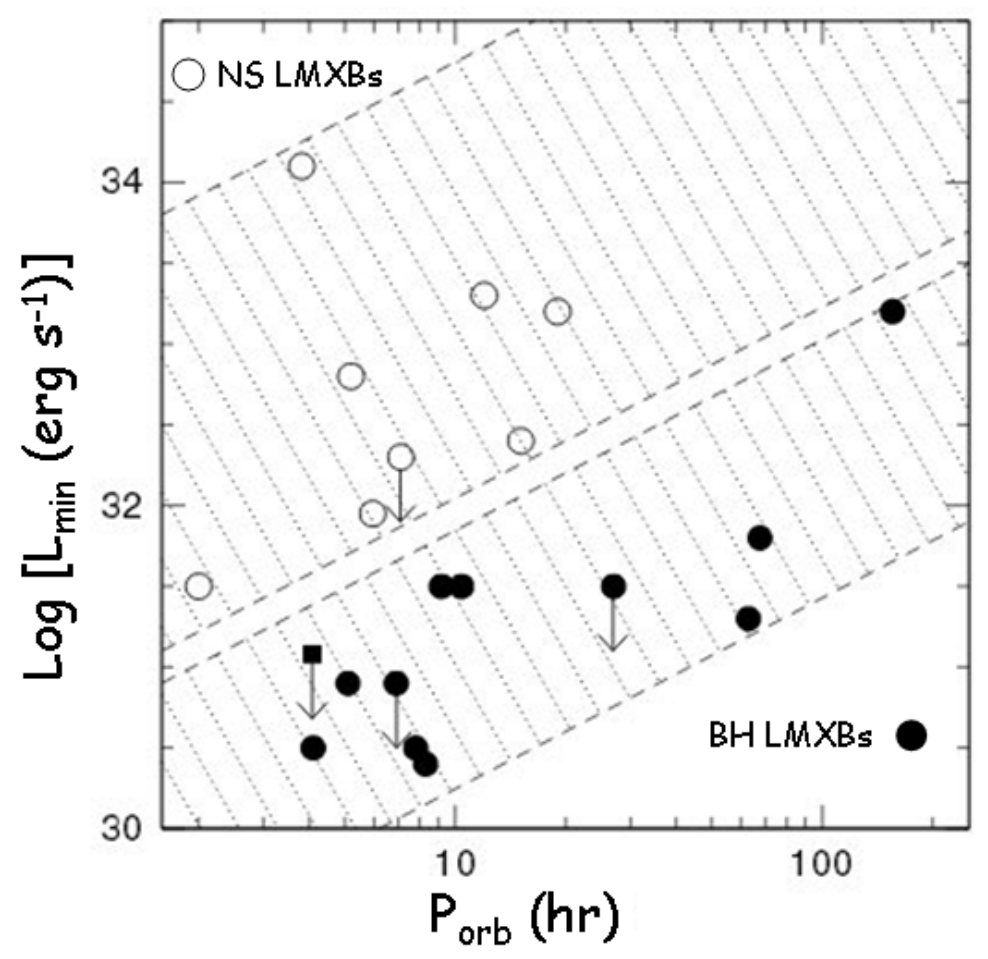

Figure 32: Minimum X-ray luminosity (0.5-10 keV) versus orbital period for BH LMXBs and NS LMXBs. The diagonal hatched areas delineate the regions occupied by the two classes of sources and indicate the dependence of luminosity on orbital period (adapted from McClintock, Narayan \& Rybicki, 2004).

It is right to remind that the mechanism proposed by GBK13 for explaining the X-ray-optical delay in A $0535+26 / \mathrm{HDE} 245770$ is based on an enhanced mass flux propagation through the viscous accretion disk. This mechanism, known as UV-optical delay (the delay of the EUV flash with respect to the optical flash) was observed and modeled for cataclysmic variables (e.g. Smak, 1984; Lasota, 2001). Time delays have been detected also in several other X-ray transient binaries. This is the reason that urged Bisnovatyi-Kogan \& Giovannelli (2017) to generalize the aforementioned model, developed for the particular case of A 0535+26/HDE 245770 (Flavia' star). This general model provides the formula (5.2) of the time delay between the optical and X-ray flashes appearance in transient cosmic accreting sources:

$$
\tau=6.9 \frac{\mathrm{m}^{2 / 3} \dot{\mathrm{m}}^{1 / 15}}{\alpha^{4 / 5}\left(\mathrm{~T}_{4}\right)^{28 / 15}}
$$

where:

$\mathrm{m}=\mathrm{M} / \mathrm{M}_{\odot} ; \quad \dot{\mathrm{m}}=\dot{\mathrm{M}} /\left(10^{-8} \mathrm{M}_{\odot} / \mathrm{yr}\right) ; \quad \mathrm{T}_{4}=\mathrm{T}_{0} / 10^{4} \mathrm{~K} ; \quad \alpha=$ viscosity, and

$\mathrm{T}_{0}=$ maximum temperature in optics.

By using this formula it is possible to obtain an excellent agreement between the experimental and theoretical delays found in: 
- X-ray/Be system A0535+26/HDE245770: $\tau_{\exp } \simeq 8$ days (GBK13); $\tau_{\text {th }} \simeq 8$ days;

- Cataclysmic variable SS Cygni; $\tau_{\text {exp }}=0.9-1.4$ days (Wheatley, Mauche \& Mattei, 2003); $\tau_{t h} \simeq 1.35$ days;

- Low-mass X-ray binary Aql X-1/V1333 Aql: $\tau_{\text {exp }} \sim 3$ days (Shahbaz et al., 1998); $\tau_{t h} \simeq$ 3.2days

- Black hole X-ray transient GRO J1655-40: $\tau_{\text {exp }} \sim 6$ days (Orosz et al., 1997); $\tau_{t h} \simeq 6.5$ days.

In this general formula the $\alpha$-viscosity parameter plays an important role, and usually it is hard to be determined. However, if the other parameters are known, because experimentally determined, the formula (5.2) can be used for determining $\alpha$, taking into account the experimental delay measured in a certain source.

Over the last couple of decades we have witnessed the discovery of a multitude of highly ionized absorbers in high-resolution X-ray spectra from both BH and NS XRBs. The first detections were obtained thanks to ASCA on the BH binaries GROJ1655-40 and GRS 1915+105. Narrow absorption lines in the spectra of these systems identified as Fe XXV and Fe XXVI indicated the first of many discoveries of photo-ionized plasmas in LMXBs (Chandra, XMM-Newton and Suzaku). Black hole hot accretion flows occur in the regime of relatively low accretion rates and are operating in the nuclei of most of the galaxies in the universe. One of the most important progress in recent years in this field is about the wind or outflow. This progress is mainly attributed to the rapid development of numerical simulations of accretion flows, combined with observations on, e.g., Sgr A ${ }^{\star}$, the SMBH in the Galactic center. The mass loss from a BH via wind is related to the mass accretion rate onto the $\mathrm{BH}$ and can be described as (Yuan, 2016):

$$
\dot{\mathrm{M}}_{\text {wind }}(\mathrm{r})=\dot{\mathrm{M}}_{\mathrm{BH}}\left(\mathrm{r} / 20 \mathrm{r}_{\mathrm{s}}\right)^{\mathrm{s}}
$$

where $\mathrm{s} \approx 1$, and $\dot{\mathrm{M}}_{\mathrm{BH}}$ is the mass accretion rate at the black hole horizon and $\mathrm{r}_{\mathrm{s}}=2 \mathrm{GM} / \mathrm{c}^{2}$ is the Schwarzschild radius.

At this point it is useful to make a sort of summary about the number of XRBSs, including CVs. Liu, van Paradijs \& van den Heuvel (2006, 2007) and Ziółkowski (2013) report 315 galactic XRBs: 197 LMXBs (63\%) and 118 HMXBs (37\%), 72 of which are Be/X-ray systems; moreover there are 62 BH candidates. Coleiro \& Chaty (2013) report that in the Milky way there are $\geq 200$ HMXBs. Ritter \& Kolb (2003) catalogue, in the 7.20 (Dec. 2013) version, reports 1166 CVs. Buckley (2015) reports about the discoveries of 530 new CVs from MASTER-Network and $855 \mathrm{CV}$ s from Catalina Real Time Susrvey (CRTS) (http://nesssi.cacr.caltech.edu/DataRelease/). Ferrario, de Martino \& Gänsicke (2015) report the number of MCVs as $\approx 250$, and $\sim 60$ of which IPs, and considering those systems for which the magnetic field intensity has not yet been determined, their number is of $\approx 600$. Table 1 shows the content of XRBSs, including CVs, in the Galaxy, and in the LMC and SMC (Ziółkowski, 2013; Ferrario, de Martino \& Gänsicke, 2015; Buckley, 2015). The mass are expressed in unit of SMC.

Grimm (2003) published: (i) a list of the 17 most luminous LMXBs contributing $\approx 90 \%$ to the integrated luminosity of LMXBs in the $2-10 \mathrm{keV}$ band in the whole Galaxy, averaged over 19962000. The 12 most luminous sources (Cir X-1, GRS 1915+105, Sco X-1, Cyg X-2, GX 349+2, 
Table 1: Comparison of numbers of different classes of X-ray Binary Systems in the Milky Way and in the Magellanic Clouds (Ziółkowski, 2013; Ferrario, de Martino \& Gänsicke, 2015; Buckley, 2015).

\begin{tabular}{|l|c|c|c|}
\hline Name of the Class & Milky Way & LMC & SMC \\
\hline $\begin{array}{l}\text { Total mass of the galaxy } \\
\text { (in MSMC units) }\end{array}$ & 100 & 10 & 1 \\
\hline High Mass X-ray Binaries & 118 & 26 & 83 \\
in this Be/X-ray & 72 & 19 & 79 \\
\hline Low Mass X-ray Binaries & 197 & 2 & - \\
\hline Black Hole Candidates & 62 & 2 & - \\
\hline Cataclysmic Variables & $\approx 2000$ & - & - \\
in this MCVs & $\approx 250$ & - & - \\
IPs & $\sim 60$ & - & - \\
B not yet determined & $\approx 600$ & - & - \\
\hline
\end{tabular}

GX 17+2, GX 5-1, GX 340+0, GX 9+1, NGC 6624, Ser X-1, GX 13+1) contribute $\approx 80 \%$ of the integrated luminosity of the Galaxy; (ii) a list of the 10 most luminous HMXBs (Cyg X-3, Cen X-3, Cyg X-1, X 1657-415, V 4641 Sgr, GX 301-2, XTE J1855-024, X 1538-522, GS 1843+009, $X 1908+075$ - that contribute $\approx 40 \%$ to the integrated luminosity of HMXBs in the $2-10 \mathrm{keV}$ band in the whole Galaxy, averaged over 1996-2000.

As already mentioned in the Section "Scenario Machine", Raguzova \& Lipunov (1999) obtained an evolutionary track that can lead to the formation of Be/BH systems. This result has been confirmed fifteen years later by Casares et al. (2014) who discovered MWC 656, the first Be/BH binary.

Indeed, Raguzova \& Lipunov (1999) calculations show that binary black holes with Be stars must have $0.2<\mathrm{e}<0.8$. It is particularly difficult to detect such systems as most of their spectroscopic variations occur in a relatively small portion of the orbit, and could easily be missed if the systems are observed at widely separated epochs.

The critical initial mass of the supernova star that collapses to a $\mathrm{BH}$ is accepted to be equal to $55<\mathrm{M}_{\mathrm{cr}}<75 \mathrm{M}_{\odot}$, and the fraction of the presupernova mass $\left(\mathrm{M}_{\star}\right)$ collapsing to the $\mathrm{BH}, \mathrm{k}_{\mathrm{BH}}=$ $\mathrm{M}_{\mathrm{BH}} / \mathrm{M}_{\star}=0.5$. The kick velocity $\mathrm{v}_{\mathrm{m}}=0-200 \mathrm{~km} \mathrm{~s}^{-1}$. The age of the system, according to their evolutionary scenario is $4 \times 10^{6} \mathrm{yr}$.

The expected number of Be/BH binaries - with orbital period $10 \mathrm{~d}<\mathrm{P}_{\text {orb }}<1000 \mathrm{~d}$, and eccentricity $0.2<\mathrm{e}<0.8-$ is $1 \mathrm{Be} / \mathrm{BH}$ for $20-30 \mathrm{Be} / \mathrm{NS}$.

Belczynski and Ziółkowski (2009) used binary population synthesis models to show that the expected ratio of Be/XRBs with neutron stars to black holes in the Galaxy is relatively high ( $30-50$ ), and so broadly in line with observations. Thus we can expect $1 \mathrm{Be} / \mathrm{BH}$ for 30-50 Be/NS.

Therefore, we can expect $1 \mathrm{Be} / \mathrm{X}$-ray BH system for 20-50 Be/X-ray NS systems (Raguzova \& Lipunov, 1999; Belczynski \& Ziółkowski, 2009). We know 60 Be/X-ray NS systems (after 
INTEGRAL). Thus we expect 1-3 Be/X-ray BH systems. One of this systems has been detected: MWC 656 (Casares et al., 2014).

New simulations - using the StarTrack binary population synthesis models have been conducted to understand the formation channel of MWC 656 - constrain the population of $\mathrm{Be} / \mathrm{BH}$ systems and study the fate of MWC 656 as a possible NS-BH merger, and then possible gravitational wave emitter. In particular, it has been assumed that all donors beyond main sequence are allowed to survive the common envelope (CE) phase. Ten Gyr of evolution of the Galactic disk originates $\sim 8700 \mathrm{~B} / \mathrm{BH}$ systems, and 1/3 of them would be Be/BH systems: namely $\sim 2900$ ). However, only 13 of them had periods, eccentricities and masses similar to MWC 656 (Grudzinska et al., 2015).

There are so many black holes in the Universe that it is impossible to count them.

Stellar-mass black holes (SBHs) form from the most massive stars when their lives end in supernova explosions. The Milky Way galaxy contains some $10^{11}$ stars. Roughly one out of every thousand stars that form is massive enough to become a black hole. Therefore, our galaxy must harbor some $10^{8}$ stellar-mass black holes. Most of these are invisible to us, and only nineteen have been identified (Wiktorowicz, Belczynski \& Maccarone, 2014) with masses up to $\sim 15 \mathrm{M}_{\odot}$. Theoretically the mass of a SBH depends on the initial mass of the progenitor, how much mass is lost during the progenitor's evolution and on the supernova explosion mechanism (Belczynski et al., 2010; Fryer et al., 2012). Mass is lost through stellar winds, and the amount of mass lost strongly depends on the metallicity of the star. For a low metallicity star $(\sim 0.01$ of the solar metallicity) it is possible to leave a black hole of $\leq 100 \mathrm{M}_{\odot}$ (Belczynski et al., 2010). In the region of the Universe visible from Earth, there are perhaps $10^{11}$ galaxies. Each one has about $10^{8}$ stellar-mass black holes. And somewhere out there, a new stellar-mass black hole is born in a supernova every second.

However, some attempts of evaluation of the number of SBHs in the Galaxy have been done. For instance, taking into account the $\gamma$-ray emissivity of the Galaxy $\left(1.3 \times 10^{43} \mathrm{~s}^{-1}\right.$ for $\mathrm{E}>100$ $\mathrm{MeV}$ ) measured by the SAS II satellite (Strong, Wolfendale \& Worral, 1976) and the processes of disk-fed accretion onto black holes, Giovannelli, Karakuła \& Tkaczyk $(1981,1982)$ found a possible upper limits to the number of black holes $\left(\mathrm{M} \sim 10 \mathrm{M}_{\odot}\right.$ and $\left.\dot{\mathrm{M}} \approx 10^{-8} \mathrm{M}_{\odot} \mathrm{yr}^{-1}\right)$ of $10^{-5}$ or $10^{-4}$ of the total star population of the Galaxy, for Mach's number 1 and 2, respectively. ${ }^{(*)}$

There is a class of intermediate-mass black holes (IMBHs), with masses $>100 \mathrm{M}_{\odot}$ up to $\approx 10^{5} \mathrm{M}_{\odot}$. It contains a dozen systems, as listed in Johnstone (2004). However, black holes with masses of several hundred to a few thousand solar masses remain elusive, as reported in a review by Casares \& Jonker (2014) where a deeply discussion about the mass measurements of SBHs and IMBHs is contained.

(*) The Mach number is given by the ratio of the velocity of the gas to the local sound speed. In order to evaluate the temperature, concentration and velocity of the plasma near the black hole it is necessary to solve the system of equations describing the plasma motion (Michel, 1972) taking into account the distance from the black hole in units of gravitational radius and the $\mathrm{u}=\mathrm{R}$-component of four velocity. Mach's number 1 and 2 correspond to different values of $\mathrm{u}_{0}^{2}(1.0266$ and 2.1213, respectively) at a distance $r_{0}$ from the black hole (Giovannelli, Karakuła \& Tkaczyk, 1981, 1982). 
Supermassive black holes (SMBHs) are $10^{6}-10^{9}$ times more massive than our Sun and are found in the centers of galaxies (see the exhaustive review by Kormendy \& Ho, 2013). Most galaxies, and maybe all of them, harbor such a black hole. So in our region of the Universe, there are some $10^{11} \mathrm{SMBHs}$. The nearest one resides in the center of our Milky Way galaxy. The most distant one we know of resides in a quasar galaxy billions of lightyears away. SMBHs grow in size as they gorge on surrounding matter.

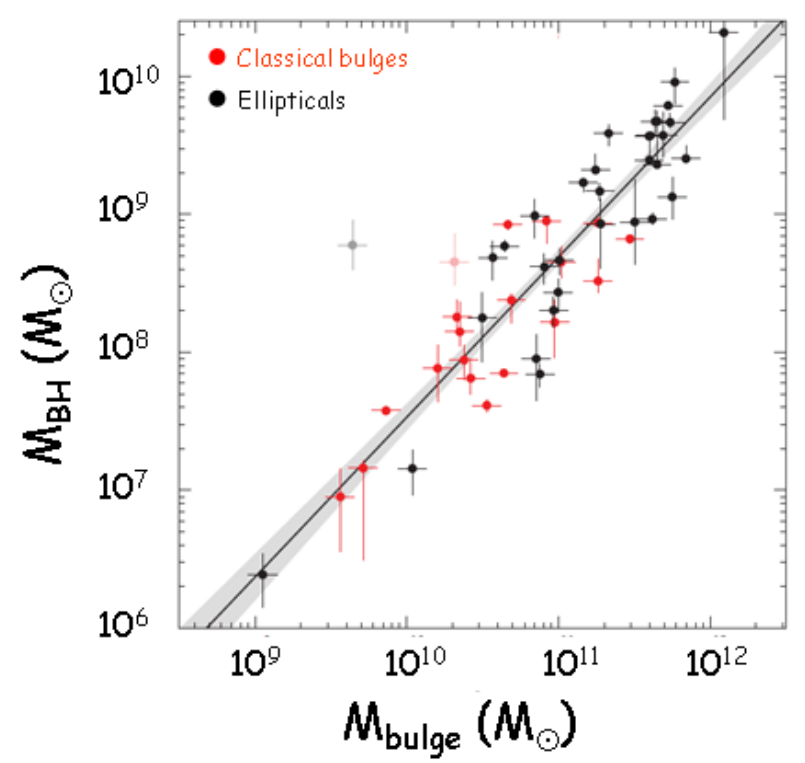

Figure 33: The correlation of the black hole mass $\left(\mathrm{M}_{\mathrm{BH}}\right)$ versus the bulge mass $\left(\mathrm{M}_{\text {bulge }}\right)$ (adopted from Kormendy \& Ho, 2013).

Figure 33 show the relative masses of super-dense cosmic objects versus the mass of the bulge (Kormendy \& Ho, 2013).

A list of BH candidates, compiled by Wm, has been reported by Robert Johnston (2004) and provides the input for constructing the map of sky locations of BH candidates, as shown in Fig. 34. Stellar-mass black holes in red, intermediate-mass black holes in purple, supermassive black holes in blue. The base image is from Tycho sky map from JPL's Solar System Simulator.

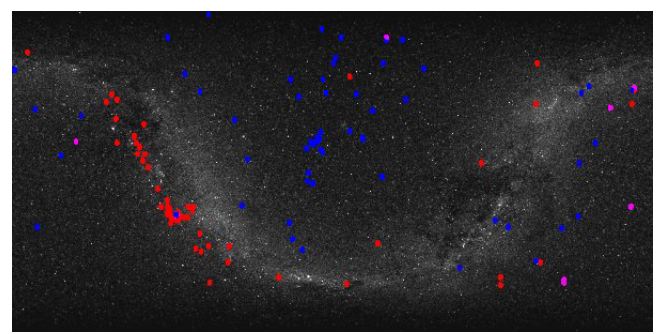

Figure 34: The map of sky locations of BH candidates. Red (stellar mass BHs), Purple (IMBHs), Blue (SMBHs). The base image is from Tycho sky map from JPL's Solar System Simulator (adopted from Johnstone, 2004). 


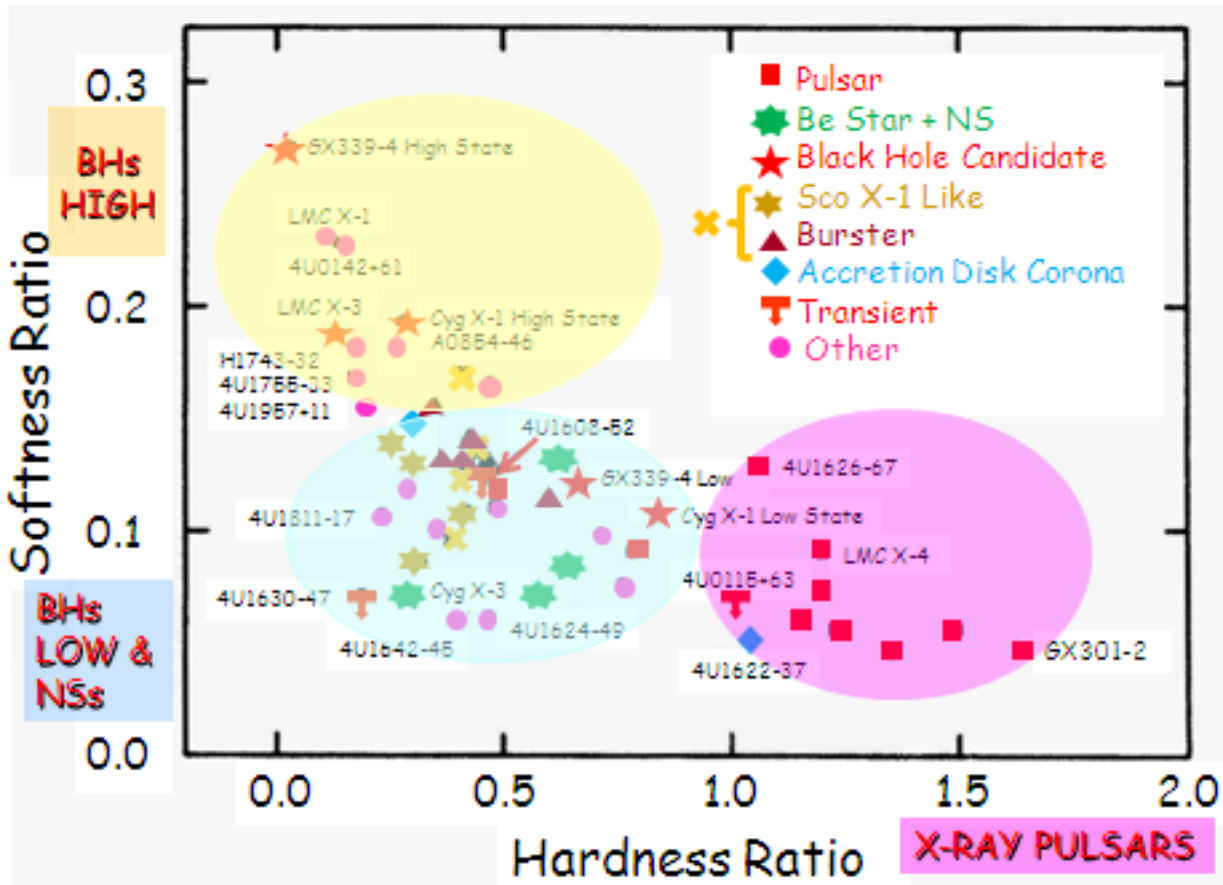

Figure 35: Softness ratio versus hardness ratio for galactic compact systems. Light yellow ellipse marks the zone where BHs in high state lie, light turquoise ellipse marks the zone of the BHs in low state and NSs, and light fuchsia ellipse marks the zone of the X-ray pulsars (adopted from Giovannelli, 2016, after Yasuo Tanaka, 2002).

In the case of galactic compact sources, by using the softness and hardness ratios, coming for the measurements of the many X-ray satellites, it is possible to construct a diagram in which BHs in high state are separated by those in low state, and by other kind of objects, such as X-ray pulsars and other systems, as shown in Fig. 35 (Giovannelli, 2016, after Tanaka, 2002).

There are several relationships in which the mass of black holes correlates with stellar dispersion velocity $\sigma$, and the bulge luminosity $\mathrm{L}_{\mathrm{V}}$, like shown in Fig. 36 (left and right panel) (McConnell et al., 2011), and the size of the disk, as shown in Fig. 37 (Mudd et al., 2018).

It is important to mention the paper by Casares (2015) in which he found that the FWHM of the $\mathrm{H} \alpha$ line in (soft X-ray transient (SXTs) is tightly correlated with the velocity semi-amplitude of the donor star $\mathrm{K}_{2}=0.233$ (13) FWHM. This new correlation, when combined with Porb (i.e., through photometric light curves), allows for the possibility of estimating compact object mass functions from single integration, low resolution spectroscopy. The correlation is the following:

$$
\mathrm{FWHM} \simeq \mathrm{A}\left(\mathrm{M}_{1} / \mathrm{P}_{\text {orb }}\right)^{1 / 3} \sin \mathrm{i}
$$

with $\mathrm{A}=876 \pm 48 \mathrm{~km} \mathrm{~s}^{-1} ; \mathrm{M}_{1}=$ Mass of the accreting star $\left(\mathrm{M}_{\odot}\right) ; \mathrm{P}_{\text {orb }}$ (days)

Figure 38 shows the $\mathrm{H} \alpha$-FWHM vs $\mathrm{K}_{2}$ correlation for SXTs with the best linear fit. Blue solid circles indicate BHs while Ns are marked by open circles (upper panel), and the $\mathrm{H} \alpha$-FWHM vs $\mathrm{K}_{2}$ correlation for CVs (lower panel). Filled triangles indicate CVs above the period gap while open triangles indicate those below/within the gap. Like in the case of SXTs, long period CVs display 

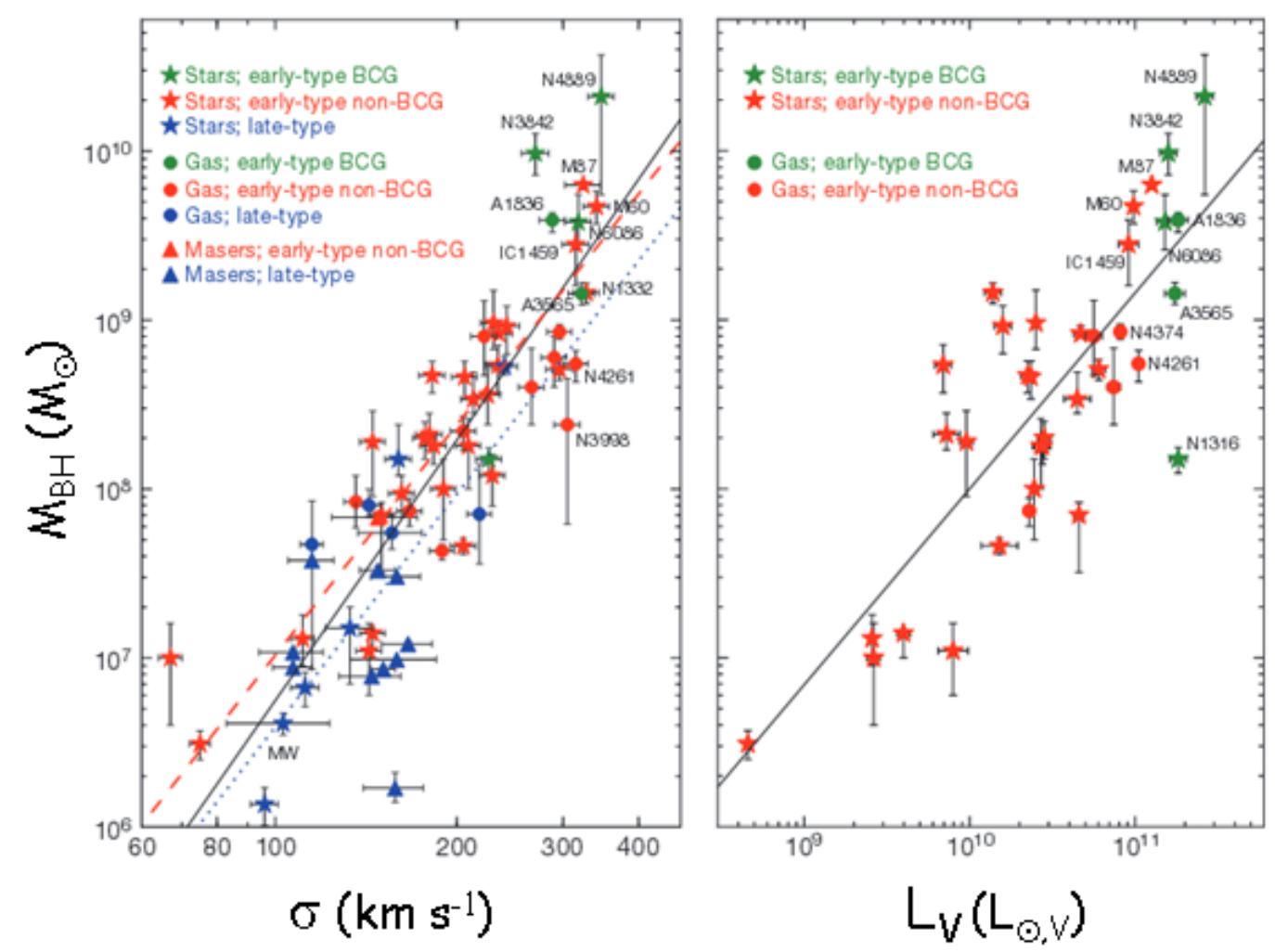

Figure 36: Correlations of dynamically measured black hole masses and bulk properties of host galaxies (adopted from McConnell et al., 2011).

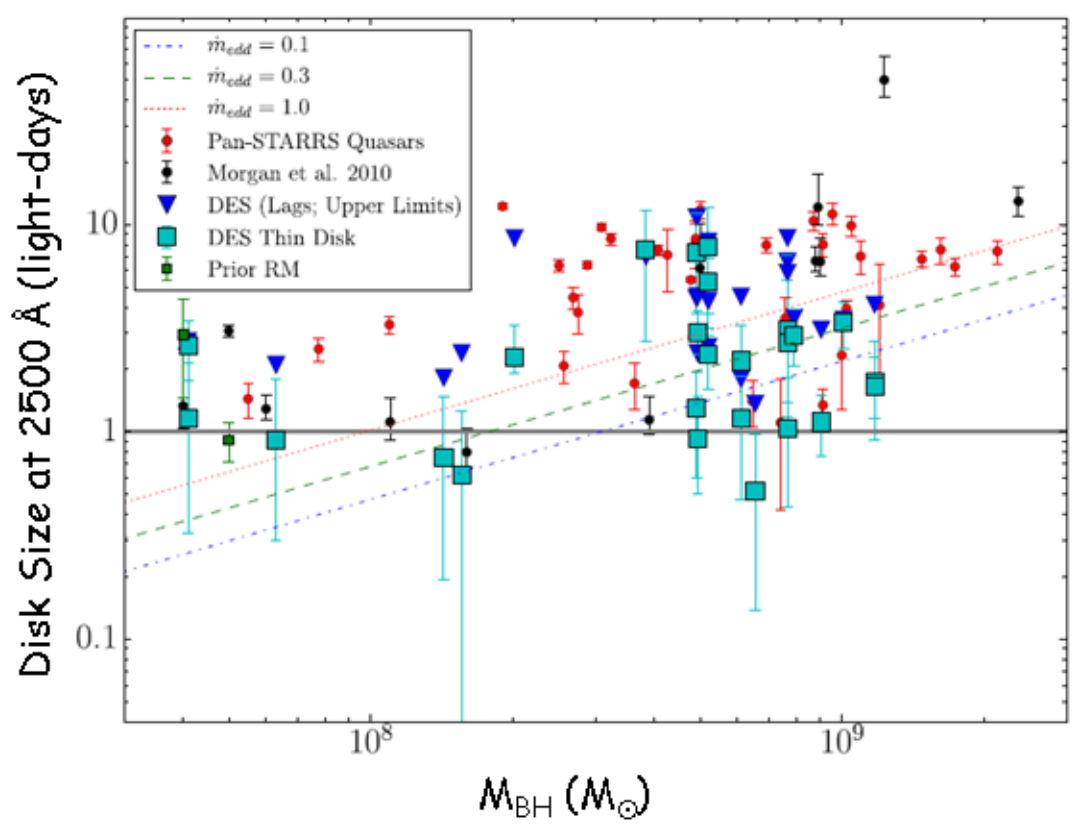

Figure 37: Disk size as a function of black hole mass (adopted from Mudd et al., 2018). 

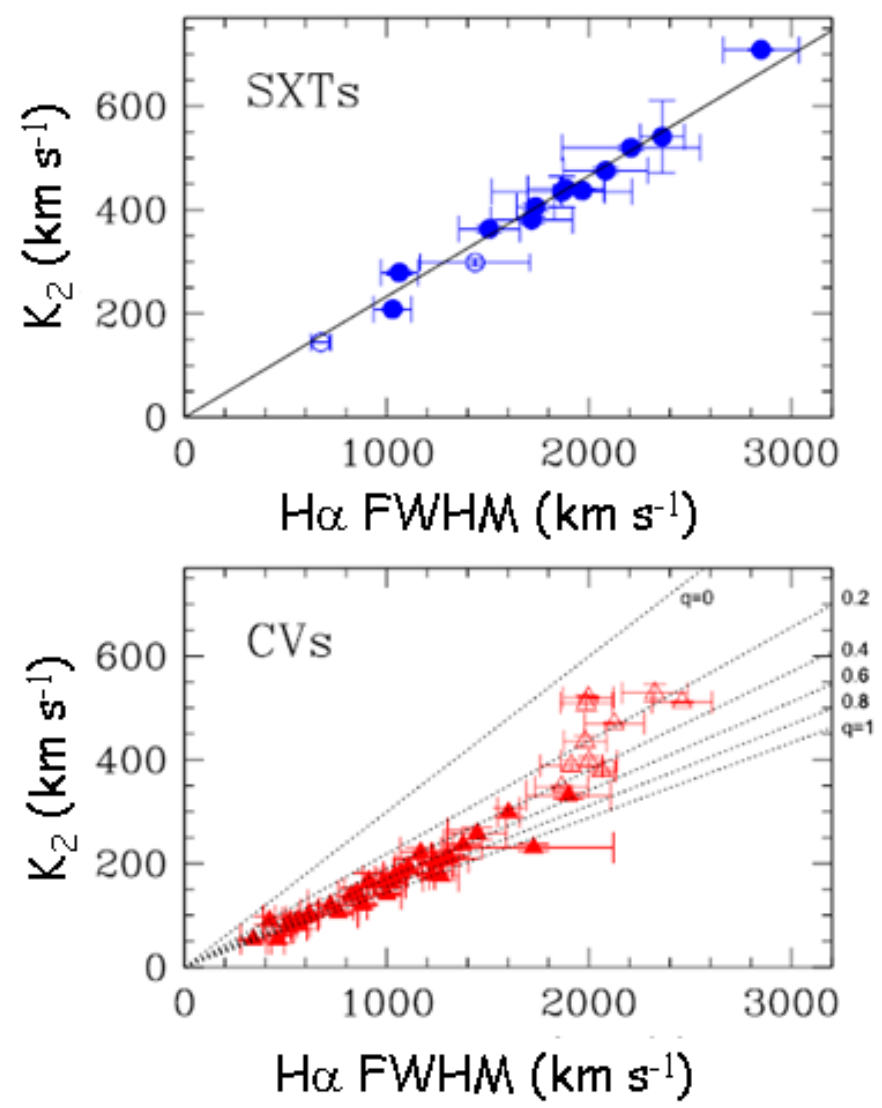

Figure 38: $\mathrm{H} \alpha$-FWHM vs $\mathrm{K}_{2}$ correlation for SXTs and CVs (adopted from Casares, 2015).

a tight $\mathrm{H} \alpha$-FWHM vs $\mathrm{K}_{2}$ correlation, though with a flatter slope. Dotted lines mark theoretical correlations derived by Casares's formulae.

Another important paper by Wu et al. (2016) reproduces the X-ray light curve of the hyperluminous X-ray source (HLX-1, whose peak X-ray luminosity $\sim 10^{42} \mathrm{erg} \mathrm{s}^{-1}$ ) near the spiral galaxy ESO 243-49, which underwent recurrent outbursts within a period of $\sim 400$ days (Fig. 39 left panel). This source is possibly the best candidate for an intermediate mass black hole (IMBH). The physical reason for this quasiperiodic variability is still unclear. Wu et al. (2016) explore the possibility of radiation-pressure instability in the accretion disk by modeling the light curve of HLX-1, and find that it can roughly reproduce the duration, period, and amplitude of the recurrent outbursts in HLX-1 with an IMBH of $\sim 10^{5} \mathrm{M}_{\odot}$. Their result provides a possible mechanism to explain the recurrent outbursts in HLX-1. They further find a universal correlation between the outburst duration and the bolometric luminosity for the black hole sources with a very broad mass range (e.g., X-ray binaries, HLX-1, and active galactic nuclei), which is roughly consistent with the prediction of radiation-pressure instability of the accretion disk (Fig. 39 right panel). These results imply that "heartbeat" oscillations triggered by radiation-pressure instability may appear in different-scale BH systems.

As already noted black hole accretion is a fundamental physical process in the universe. It is 

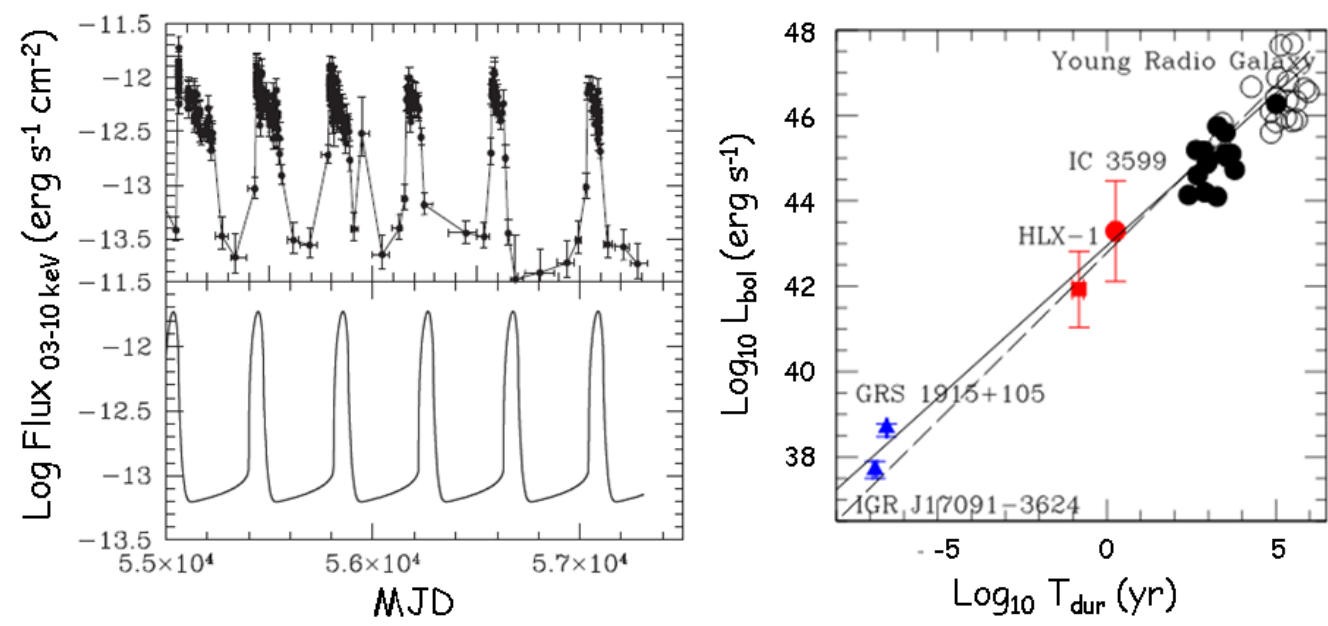

Figure 39: Left panel shows the observational light curve of HLX-1 (top panel) and modeled light curve of accretion disk (bottom). Right panel shows Correlation between the bolometric luminosity and the outburst duration for different-scale BHs. The black filled and empty circles represent the Gigahertz-peaked spectrum (GPS) and compact steep-spectrum (CSS) sources, respectively. The solid line represents the best fit and the dashed line shows the prediction of the disk model under radiation-pressure instability $(\alpha=0.02)$ (adopted from $\mathrm{Wu}$ et al., 2016).

the standard model for the central engine of active galactic nuclei (AGNs), and also plays a central role in the study of black hole X-ray binaries, Gamma-ray bursts, and tidal disruption events. According to the temperature of the accretion flow, the accretion models can be divided into two classes, namely cold and hot. The standard thin disk model belongs to the cold disk, since the temperature of the gas is far below the virial value (Shakura \& Sunyaev, 1973) (see reviews by (Pringle, 1981; Frank, King \& Rayne, 2002; Abramowicz \& Fragile, 2013; Blaes, 2014). The disk is geometrically thin but optically thick and radiates multi-temperature black body spectrum. The radiative efficiency is high, $\sim 0.1$, independent of the accretion rate. The model has been successfully applied to luminous sources such as luminous AGNs and black hole X-ray binaries in the thermal state. The most recent review about the accretion onto black holes was published by Lasota (2016).

The tidal disruption of stars by massive BHs has been discussed since many years by Rees (1988b), and e.g. Magorrian \& Tremaine (1999). Rees (1988b) argued that stars in galactic nuclei can be captured or tidally disrupted by a central black hole. Some debris would be ejected at high speed, the remainder would be swallowed by the hole, causing a bright flare lasting at most a few years. Such phenomena are compatible with the presence of $10^{6}-10^{8} \mathrm{M}_{\odot}$ holes in the nuclei of many nearby galaxies. Stellar disruption may have interesting consequences in our own Galactic Centre if $\mathrm{a} \approx 10^{6} \mathrm{M}_{\odot}$ hole lurks there.

In a recent paper, Bisnovatyi-Kogan \& Giovannelli (2017) developed models of time lags between optical and X-ray flashes for close-binary galactic sources with accretion disks and for an AGN with an SMBH that is embedded in a quasi-spherical bulge. The flashes in an AGN are considered in the model when a disruption of a star that is in the evolution phase of a giant enters the 
radius of strong tidal forces. The matter with a low angular momentum that is released by the star falls into the SMBH in the form of a quasi-spherical flow with a velocity that is close to the free-fall velocity. An X-ray flash occurs when the falling matter reaches the hot inner regions. The time lag observed in these sources is identified with the time of the matter falling from the tidal radius onto the central region. The values of the tidal radius that they calculated in this model were compared with the theoretical radii of a tidal disruption that depends on the masses of the SMBH and of the star, and on the radius of the star. Knowing the SMBH masses from observations, and making a reasonable suggestion for the stellar mass that is on the order of one solar mass, they obtained that the radii of the disrupted star are between a few tens and a few hundreds of $\mathrm{R}_{\odot}$. These radii are characteristic of stars of moderate mass on the giant phase of evolution (e.g. Bisnovatyi-Kogan, 2011).

Recently several books about black holes appeared in the literature:

- The Physics of Accretion onto Black Holes (Falanga et al., 2015). It provides a comprehensive summary on the physical models and current theory of black hole accretion, growth and mergers, in both the supermassive and stellar-mass cases.

- The Strongest Magnetic Fields in the Universe (Beskin et al. 2015). The topic of this volume contains reviews of completely new aspects of magnetic fields in the astrophysical Universe. The strength of the magnetic fields of the compact objects reviewed in this volume is up to 8 to 10 orders of magnitude higher than that of typical sunspots which are the strongest fields in the solar system. Large-scale astrophysical magnetic fields, such as interstellar and galactic fields are weaker still than the fields experienced in the solar system.

- The Formation and Disruption of Black Hole Jets (Contopoulos, Gabuzda \& Kylafis, 2015). The main aim of this book is to present reviews of the varied phenomenology regarding the radio to X-ray spectra of stellar binaries and the properties of AGN jets on the wide range of scales through which they propagate, as well as recent theoretical efforts to understand the physical mechanisms that contribute to the origin of black hole jets on all scales. Particular emphasis is given to the role and the origin of the black hole magnetosphere and the magnetic fields that drive, collimate, and accelerate the jets. The final goal is a tentative of giving a consistent, unified physical picture of the formation and disruption of jets in accreting black hole systems. New observational and theoretical results are piling up every day, so the contents of this volume only represent our current best ideas. Time will tell how close our present understanding is to reality.

- Astrophysics of Black Holes (Bambi, 2016). This book contains the most relevant papers discussed during the Fudan Winter School on Astrophysical Black Holes (held at Fudan University, in Shanghai, from February 10 to 15, 2014): Black Hole Accretion Discs, Transient Black Hole Binaries, Black Hole Spin: Theory and Observation, Winds from Black Hole Accretion Flows: Formation and Their Interaction with ISM, A Brief Review of Relativistic Gravitational Collapse, and General Relativity in a Nutshell.

- Black Holes: A Laboratory for Testing Strong Gravity (Bambi, 2017a). The main aim of this book is to discuss the electromagnetic techniques to study the strong gravity region 
around astrophysical black holes. For completeness, gravitational wave methods will be also reviewed, but only very briefly and without the necessary details to start working on the corresponding line of research. This book has not the ambition to be a complete manual on this research field. Hopefully, it may be a good starting point.

Important news were reported over the last couple of decades. We have witnessed the discovery of a multitude of highly ionized absorbers in high-resolution X-ray spectra from both $\mathrm{BH}$ and NS XRBs. The first detections were obtained thanks to ASCA on the BH binaries GROJ165540 and GRS 1915+105. Narrow absorption lines in the spectra of these systems identified as Fe $\mathrm{XXV}$ and Fe XXVI indicated the first of many discoveries of photo-ionized plasmas in LMXBs (Chandra, XMM-Newton and Suzaku).

\section{Gravitational waves}

The Universe that contains by definition all the matter or all the energy available showed one important event that was possible to be detected on the Earth. This event was a further direct experimental demonstration of the validity of the GR theory. Indeed, on September 14, 2015 at 09:50:45 UTC the two detectors of the Laser Interferometer Gravitational-Wave Observatory (LIGO) simultaneously observed a transient gravitational-wave signal. It matches the waveform predicted by GR theory for the inspiral and merger of a pair of black holes and the ringdown of the resulting single black hole. The signal was observed with a significance $\geq 5.1 \sigma$ (Fig. 40). The source lies at a luminosity distance of $410_{-180}^{+160} \mathrm{Mpc}$ corresponding to a redshift $\mathrm{z}=0.090_{-0.04}^{+0.03}$. In the source frame, the initial black hole masses are $36_{-4}^{+5} \mathrm{M}_{\odot}$ and $29 \pm 4 \mathrm{M}_{\odot}$, and the final black hole mass is $62 \pm 4 \mathrm{M}_{\odot}$ with $3.0 \pm 0.5 \mathrm{M}_{\odot} \mathrm{c}^{2}$ radiated in gravitational waves. All uncertainties define $90 \%$ credible intervals. These observations demonstrate the existence of binary stellar-mass black hole systems. This is the first direct detection of gravitational waves and the first observation of a binary black hole merger (Abbott et al., 2016a).

Abbott et al. (2016b) reported the second observation of a gravitational-wave signal produced by the coalescence of two stellar-mass black holes. The signal, GW151226, was observed by the twin detectors of the LIGO on December 26, 2015 at 03:38:53 UTC. The signal was detected at significance $\geq 5 \sigma$. The inferred source-frame initial black hole masses are $14.2_{-3.7}^{+8.3} \mathrm{M}_{\odot}$ and $7.5 \pm$ $2.3 \mathrm{M}_{\odot}$, and the final black hole mass is $20.8_{-1.7}^{+6.1} \mathrm{M}_{\odot}$. We find that at least one of the component black holes has spin greater than 0.2 . This source is located at a luminosity distance of $440_{-190}^{+180}$ Mpc corresponding to a redshift $\mathrm{z}=0.09_{-0.04}^{+0.03}$. All uncertainties define a $90 \%$ credible interval. This second gravitational-wave observation provides improved constraints on stellar populations and on deviations from the GR theory.

For these detections of gravitational waves - first predicted by Einstein 100 years ago - Rainer Weiss, Barry Barish \& Kip Thorne have been awarded the 2017 Nobel prize in physics.

Abbott et al. (2016c) present a possible observing scenario for the Advanced LIGO (aLIGO) and Advanced Virgo gravitational-wave detectors over the next decade, with the intention of providing information to the astronomy community to facilitate planning for multi-messenger astronomy with gravitational waves.

Gravitational waves provide a revolutionary tool to investigate yet unobserved astrophysical objects. Especially the first stars, which are believed to be more massive than present-day stars, 


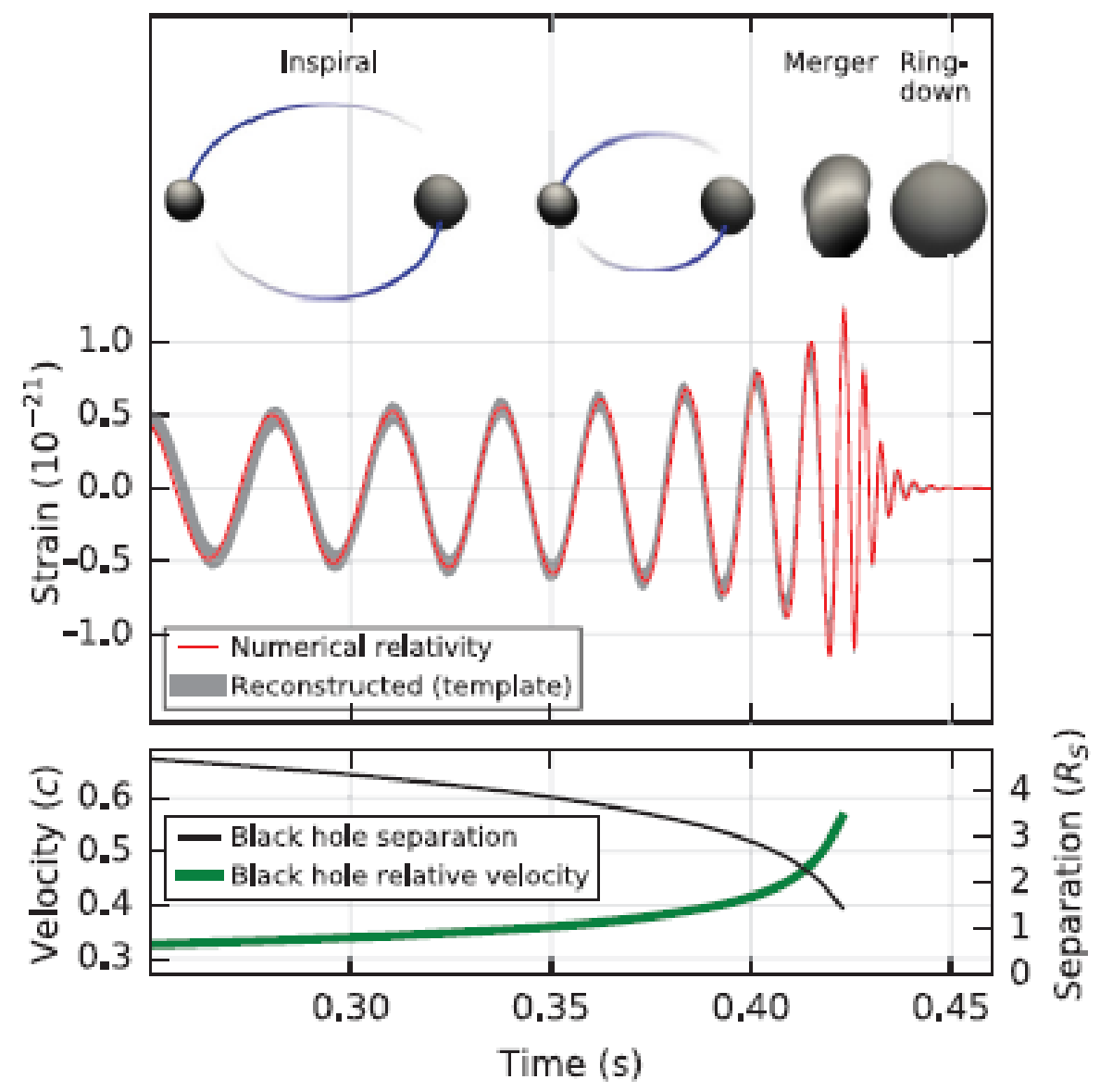

Figure 40: The GW150914 event. Top: estimated gravitational-wave strain amplitude. Bottom: the Keplerian effective black hole separation in units of Schwarzschild radii (adopted from Abbott et al., 2016a).

might be indirectly observable via the merger of their compact remnants. An interesting paper by Hartwig et al. (2016) developed a self-consistent, cosmologically representative, semi-analytical model to simulate the formation of the first stars. They estimated the contribution of primordial stars to the merger rate density and to the detection rate of the aLIGO. Owing to their higher masses, the remnants of primordial stars produce strong GW signals, even if their contribution in number is relatively small. They found a probability of $\geq 1 \%$ that the current detection GW150914 is of primordial origin. The higher masses of the first stars boost their GW signal, and therefore their detection rate. Up to five detections per year with aLIGO at final design sensitivity originate from Pop III BH-BH mergers. Approximately once per decade, we should detect a BH-BH merger that can unambiguously be identified as a Pop III remnant.

On 2017 August 17 the merger of two compact objects with masses consistent with two neutron stars was discovered through gravitational-wave (GW170817), gamma-ray (GRB 170817A), and optical (SSS17a/AT2017gfo) observations. The optical source was associated with the earlytype galaxy NGC 4993 at a distance of just $\sim 40 \mathrm{Mpc}$, consistent with the gravitational-wave measurement, and the merger was localized to be at a projected distance of $\sim 2 \mathrm{kpc}$ away from the galaxy's center (Abbott et al., 2017a,b). 
Lipunov et al. (1995) predicted the NS-NS merger at a distance of $\leq 50 \mathrm{Mpc}$ and the possibility of detecting GWs!

This prediction was born by the "Scenario Machine" that describes the evolution of gravimagnetic rotators (Lipunov, 1987; Lipunov, \& Postnov, 1988), and recently commented by Giovannelli (2016).

On August 17, 2017 Multimessenger Astrophysics born! As pioneer of the Multifrequency Astrophysics, I am particularly happy!

The Gravitational-Wave Transient Catalog of Compact Binary Mergers Observed by LIGO and Virgo during the First and Second Observing Runs contains 11 events: 10 BH-BH mergers and 1 NS-NS merger (LIGO-VIRGO Gravitational Wave Open Science Center, 2018) at https://www.gwopenscience.org/catalog/GWTC-1-confident/html/.

\section{What intelligent humanity is doing?}

As I already wrote in the first section, in this era - very troubled by major events, such as the overpopulation of the planet, the unstoppable migratory flows, due to the looting of the poorest countries, the run-up to nuclear weapons in the false illusion that peace can be guaranteed by weapons, poisoning apparently unstoppable of the planet - humanity is going along a wrong path.

Indeed, unfortunately, the alarms of an imminent disaster are there for all to see. The temperature of the planet is increasing dramatically, as shown in Fig. 41 (Saraceno, 2012), and this can cause irreparable damage to the survival of the human species and beyond.

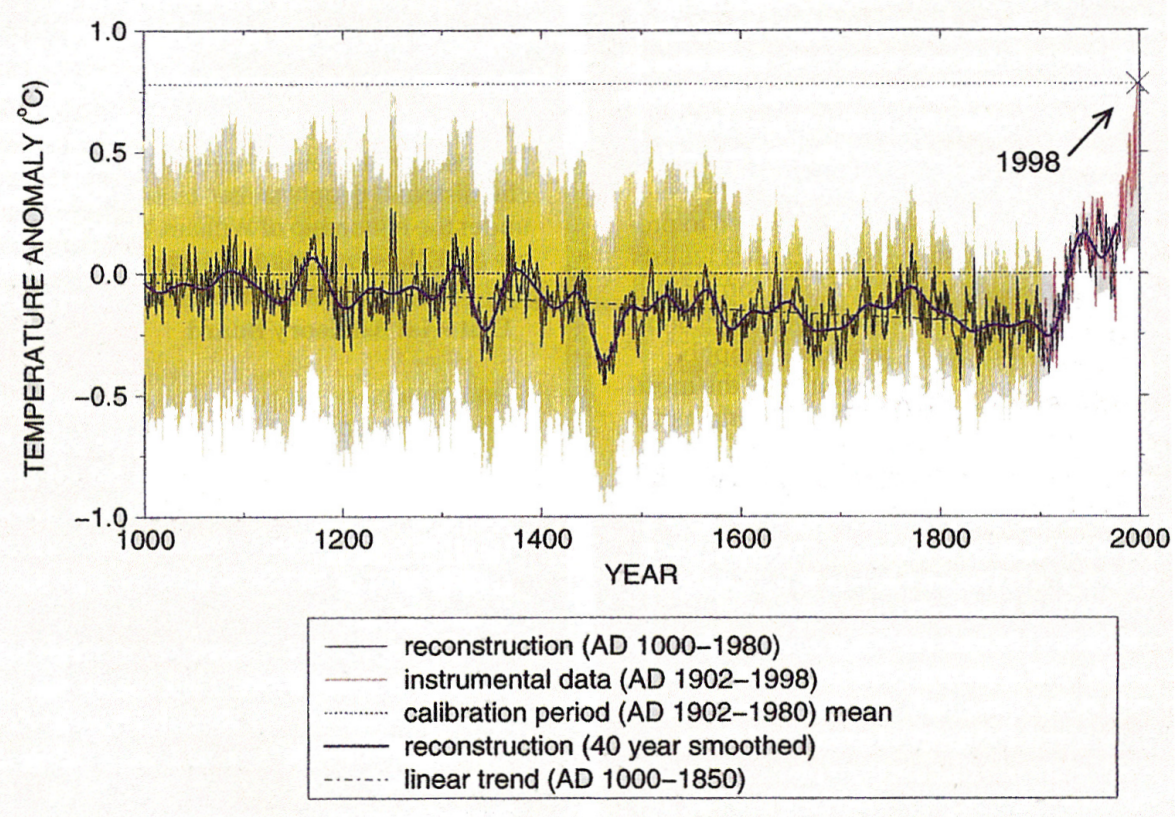

Figure 41: The temperature of the Earth from the year 1000 A.C. (adopted from Saraceno, 2012).

Indeed, for instance, the 2017 Hurricane Season Really Is More Intense Than Normal, like shown in Fig. 29. There have been 13 named storms in 2017. Only four other seasons since 1995 
have had that many by Sept. 18. Just two more by the end of the year would put 2017 in the top 15 since 1851, when reliable records begin (Astor, 2017).

The winds of such hurricanes are extremely high, e.g.: Harvey $\left(\mathrm{v}_{\max }=215 \mathrm{~km} / \mathrm{h}\right), \operatorname{Irma}\left(\mathrm{v}_{\max }\right.$ $=285 \mathrm{~km} / \mathrm{h})$, Jose $\left(\mathrm{v}_{\max }=250 \mathrm{~km} / \mathrm{h}\right)$, Maria $\left(\mathrm{v}_{\max }=280 \mathrm{~km} / \mathrm{h}\right)$, and the tropical storm Katia $\left(\mathrm{v}_{\max }=\right.$ $120 \mathrm{~km} / \mathrm{h}$ ) (Wikipedia, 2017). It is superfluous to remind that the energy is proportional to $\mathrm{v}_{\text {max }}^{2} ! ! !$ And the most important effects are evaluated in the provoked "damage" in U.S. dollars, without admitting that the effects of such hurricanes are son of the "stupidity" of humans!!!

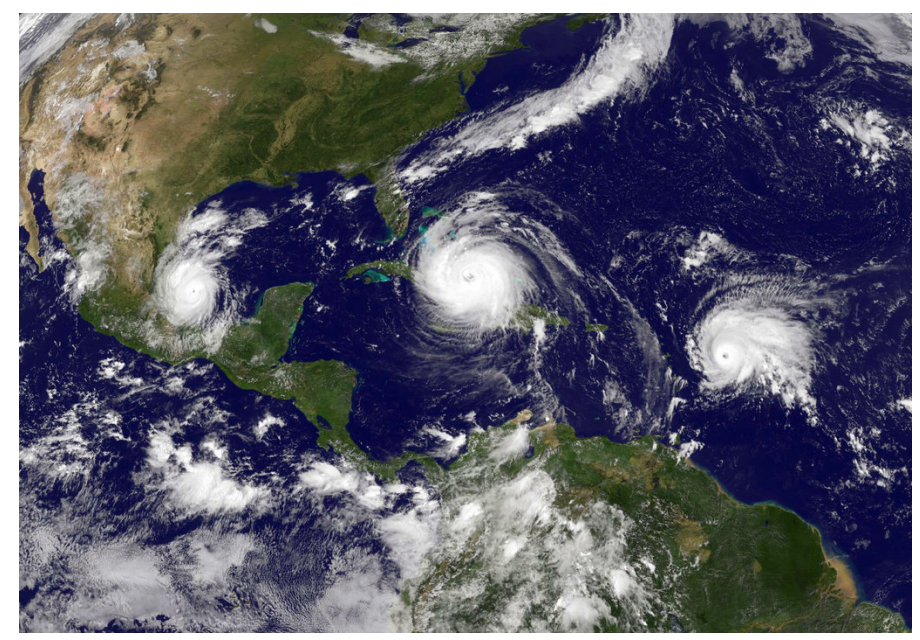

Figure 42: On Sept. 8, 2017 three storms threatened land: from left, Tropical Storm Katia, Hurricane Irma and Tropical Storm Jose, which would intensify into a hurricane (Adopted from Astor, 2017 - Credit: NOAA/NASA GOES Project).

In this regard, see the article by Giora Shaviv (2016). He discusses the phenomenon called Global Warming or in the new name Climate Change that refers to the behavior of the 'Temperature of the Earth' as a function of time. The 'temperature of the Earth' is a complicated term as it is measured in different locations and one has to define at what location and how to average the temperature over the complicated surface of the Earth so as to obtain a single number. The atmosphere, or better said the troposphere, can be considered as a boundary layer. However, this layer is not isothermal and shows large temporal and location temperature variations. The conclusions are not given because the problem is so complicated that the numerous theoretical models lead to significantly different results from each other.

\subsection{Earth surface loss}

Following the synthesis of Pier Luigi Luisi (2018) from 1950 till today:

- we have destroyed one half of the earth forests;

- we have inserted in the atmosphere large quantities of $\mathrm{CO}_{2}$, methane and other polluting substances;

- we have increased the average temperature of the planet of $\sim 0.5^{\circ} \mathrm{C}$, which can go up to $\gtrsim$ $2^{\circ} \mathrm{C}$; 
- we created a large ozone hole;

- $\sim 65 \%$ of land, once good for cultivation, is no longer available to farmers;

- and only $\sim 12 \%$ of earth surface is left for the conservation of non human species;

- the river has been contaminated by industrial waste;

- we have a loss of biodiversity about thousand time higher than normal;

- we continue to produce nuclear waste;

- almost one third of the world population lives with less than a dollar/day

\subsection{Accretion processes onto oceans}

A huge, swirling pile of trash in the Pacific Ocean is growing faster than expected and is now about three times the size of France (https://www.theoceancleanup.com/great-pacific-garbagepatch/). An astonishing partial image of this disaster is shown in Fig. 43 (left panel) (http://www.eio ba.pl/a/4f00/zabije-nas-plastik). The right panel of Fig. 43 shows a sea turtle entangled in a ghost net (Photo credits: Francis Perez).
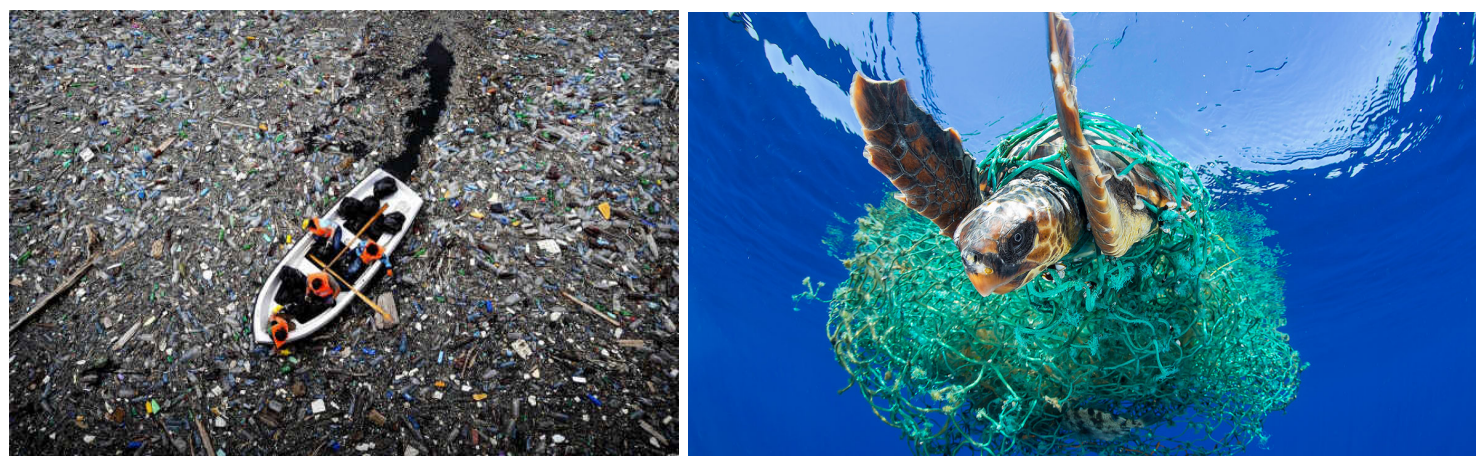

Figure 43: Left panel: partial image of the swirling pile of trash in the Pacific Ocean. Right panel: A sea turtle entangled in a ghost net (Photo credits: Francis Perez).

Our trash harms the deepest fish in the ocean. Humans have produced $8.3 \times 10^{12} \mathrm{~kg}$ of plastic since the ' 50 s (Geyer, Jambeck \& Law, 2017). That is equal in weight to $\approx 1.6 \times 10^{9}$ elephants, considering the average weight of an elephant of $\approx 5000 \mathrm{~kg}$ (https://www.livescience.com/27320elephants.html).

Garbage patches are large areas of the ocean where litter, fishing gear, and other debris known as marine debris - collects. They are formed by rotating ocean currents called "gyres". There are five gyres in the oceans. One in the Indian Ocean, two in the Atlantic Ocean, and two in the Pacific Ocean. Garbage patches of varying sizes are located in each gyre. The most famous of these patches is often called the "Great Pacific Garbage Patch". It is located in the North Pacific Gyre (between Hawaii and California). "Patch" is a misleading nickname, causing many to believe that these are islands of trash. Instead, the debris is spread across the surface of the water and from the surface all the way to the ocean floor. The debris ranges in size, from large abandoned 
fishing nets to tiny microplastics, which are plastic pieces smaller than $5 \mathrm{~mm}$ in size. This makes it possible to sail through some areas of the Great Pacific Garbage Patch and see very little to no debris (https://marinedebris.noaa.gov/info/patch.html).

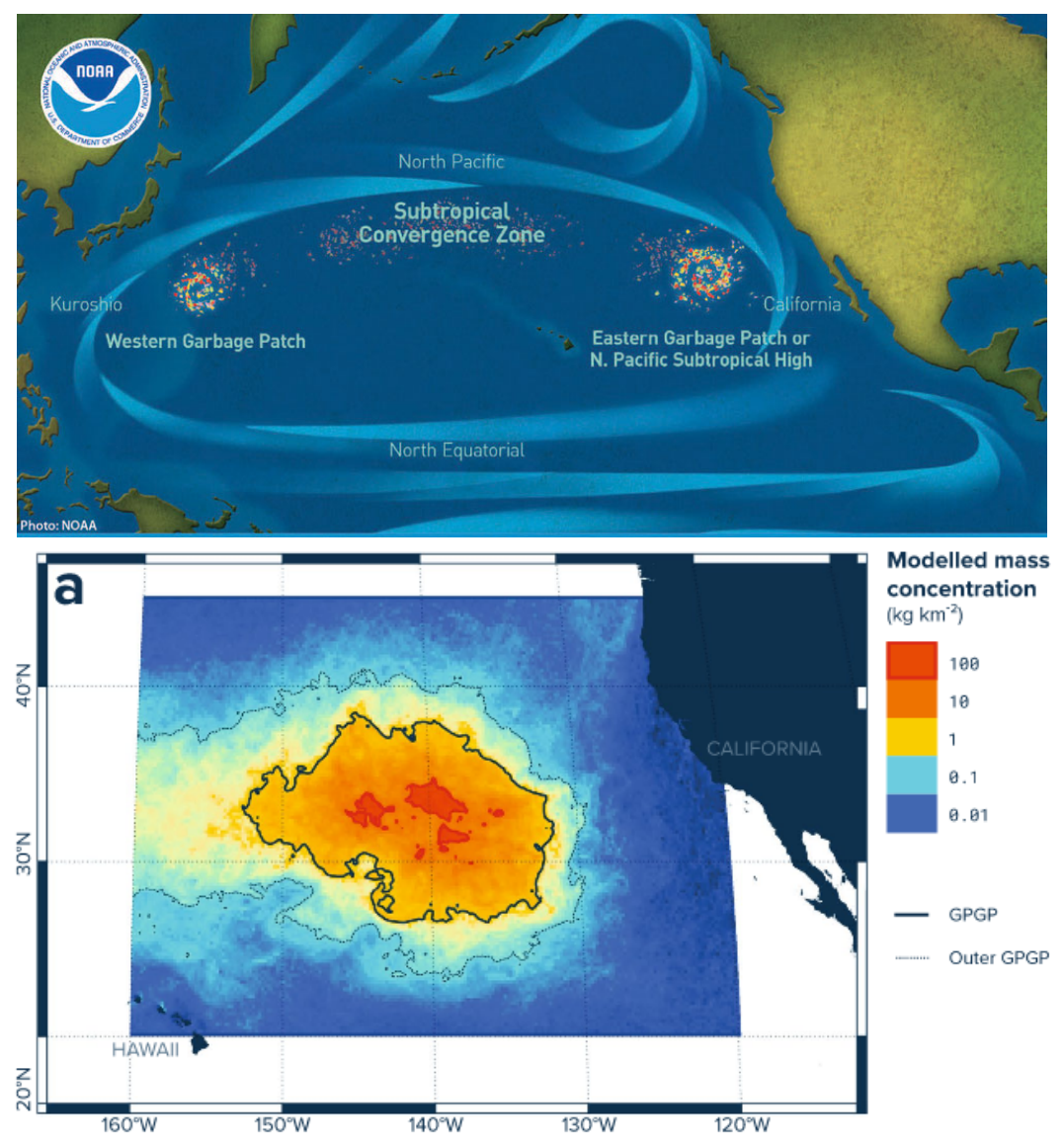

Figure 44: The great pacific garbage patch: upper panel - the extension (Map by NOAA); lower panel - the model (adopted from Lebreton et al., 2018).

Figure 44 (upper panel) shows the dramatic extension of the great pacific garbage patch (NOAA, Marine Debris Program, 2012 - https://marinedebris.noaa.gov/info/patch.html. However, a paper describing the dramatic situation of the garbage has been published by Lebreton et al. (2018). They state that ocean plastic can persist in sea surface waters, eventually accumulating in remote areas of the world's oceans. They characterize and quantify a major ocean plastic accumulation zone formed in subtropical waters between California and Hawaii: The Great Pacific Garbage Patch (GPGP). Their model, calibrated with data from multi-vessel and aircraft surveys, predicted at least $79_{-34}^{+50}$ thousand tonnes of ocean plastic are floating inside an area of $1.6 \times 10^{6} \mathrm{~km}^{2}$; a figure four to sixteen times higher than previously reported (Fig. 44, lower panel). They explain this difference through the use of more robust methods to quantify larger debris. Over three-quarters of the GPGP mass was carried by debris larger than $5 \mathrm{~cm}$ and at least $46 \%$ was comprised of fishing nets. Microplastics accounted for $8 \%$ of the total mass but $94 \%$ of the estimated $1.8_{-0.7}^{+1.8} \times 10^{9}$ pieces floating in the area. Plastic collected during their study has specific characteristics such as small surface-to-volume ratio, indicating that only certain types of debris have the capacity to persist and 


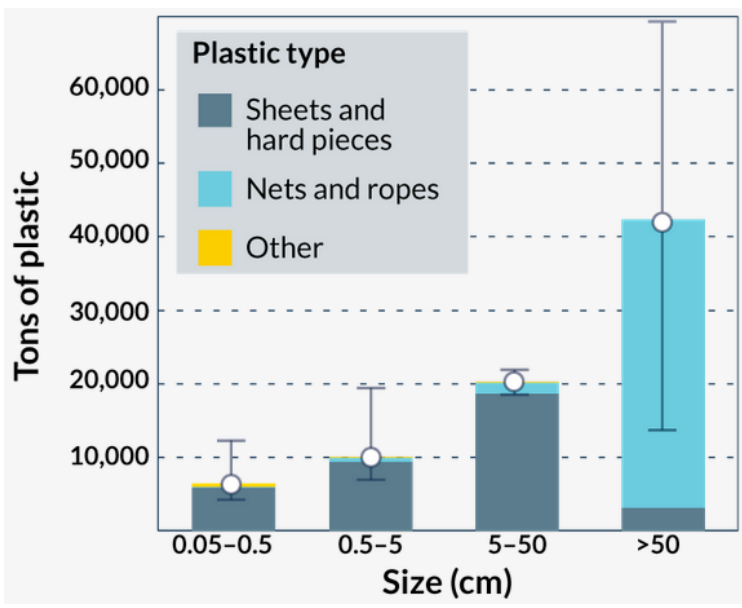

Figure 45: Inventory of plastic in the great pacific garbage patch (GPGP) (adopted from Lebreton et al., 2018).

accumulate at the surface of the GPGP. Finally, their results suggest that ocean plastic pollution within the GPGP is increasing exponentially and at a faster rate than in surrounding waters.

Figure 45 shows an inventory of plastic in the GPGP (Lebreton et al., 2018).

\subsection{Accretion processes in the space surrounding the Earth}

The Soviet Union launched the first space vehicle Sputnik I on October 4, 1957 (Stoiko, 1970). Since then, the exploration and use of space have proliferated dramatically. Activities in space have generated millions of pounds of debris, most of it remaining in Earth orbit. This debris poses a threat to individuals and equipment moving in space. On rare occasions, the debris also threatens the Earth's biosphere (Roberts, L.D. 1992). This author discusses the global legal regime that currently regulates outer space comprises four international agreements. This regime consists of the Treaty on Principles Governing the Activities of States in the Exploration and Use of Outer Space, Including the Moon and Other Celestial Bodies (Outer Space Treaty), the Convention on International Liability for Damage Caused by Space Objects (Liability Convention), the Convention on Registration of Objects Launched into Outer Space (Registration Convention), and the Agreement Governing the Activities of States on the Moon and Other Celestial Bodies (Moon Treaty). Although covering a wide range of issues relating to outer space, these agreements (collectively, Outer Space Agreements or Agreements) have not effectively managed the problem of space debris. Moreover, the Agreements are partly responsible for creating the problem.

The Outer Space Agreements were intended primarily to facilitate access to and use of the space environment, although they also included elements of environmental regulation. The Agreements typically raised environmental concerns only in the context of efficient use of space resources or research opportunities. They did not attempt to provide broader protection of the space environment. These factors, as well as the complexities of the space environment, make it difficult for the Agreements to provide a solution to the continuing degradation of the space environment.

This degradation, as well as the pollution of the entire planet, has reached alarming levels, as shown schematically in Figure 46 (upper panel) (Rayne Fisher-Quann, 2017 at https://quarkmag.co 
m/is-space-junk-a-problem-67e865f702a9). Lower panel of Fig. 46 shows John Glenn's disconsolate statement in a cartoon (Credit: Joe Heller).

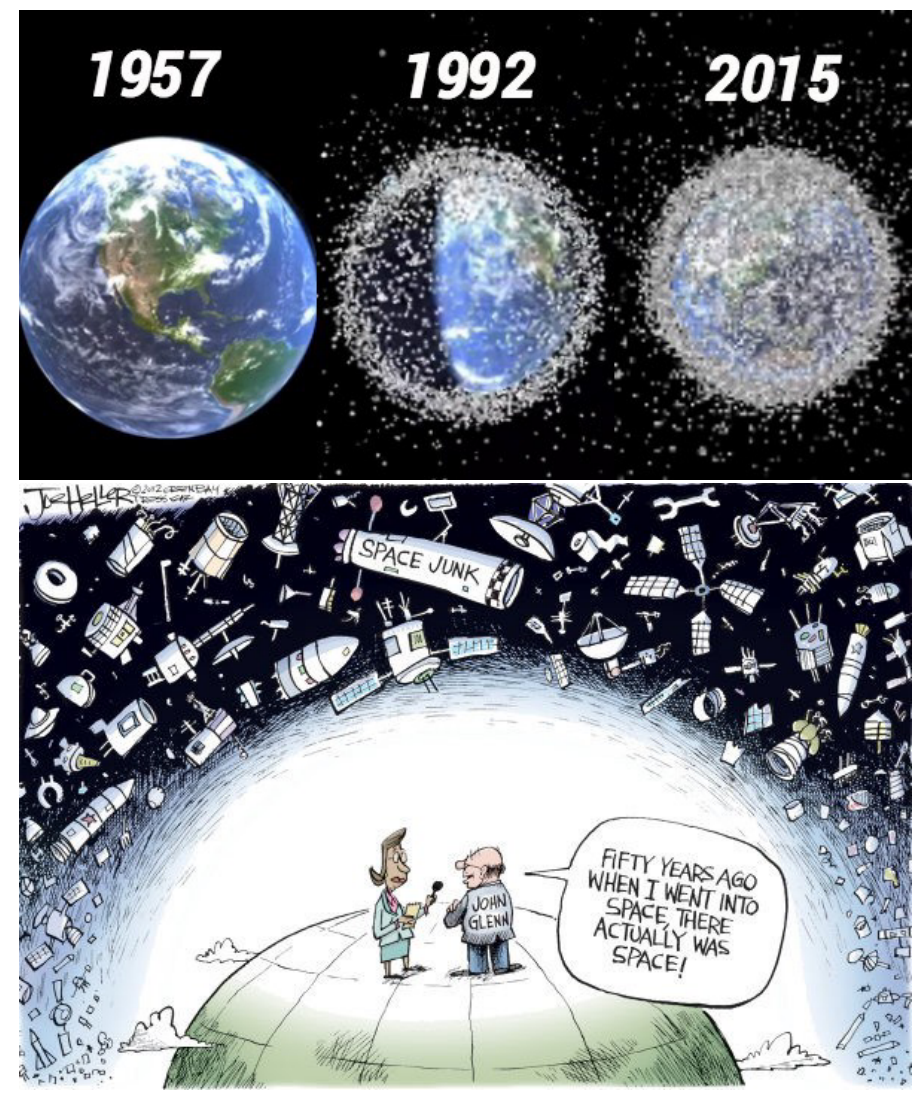

Figure 46: Upper panel: the amount of space junk in our atmosphere has skyrocketed, with dangerous consequences (Fisher-Quann, 2017). Lower panel: John Glenn's disconsolate statement (Credit: Joe Heller).

More than 500,000 pieces of debris, or "space junk", are tracked as they orbit the Earth. They all travel at speeds up to $28,000 \mathrm{~km} \mathrm{~h}^{-1}$, fast enough for a relatively small piece of orbital debris to damage a satellite or a spacecraft.

The rising population of space debris increases the potential danger to all space vehicles, but especially to the International Space Station, space shuttles and other spacecraft with humans aboard.

Indeed, currently there is about six metric tons of space debris in earth orbit and about $45 \%$ of that is in low-earth orbit and polar orbits where the threat of collisions continues to increase. This process can lead to an escalating cascade of more and more debris. Today we are very much at risk of such a cascading build-up that is known as the "Kessler Syndrome". Two events in recent years have particularly contributed to orbital space debris build-up. One event was the collision of the defunct Russian Kosmos 2251 weather satellite with the Iridium 33 low earth orbit mobile communications satellite. The other was the shooting down of an old and defunct Chinese Fen Yun weather satellite by the Chinese military. Each of these events led to the creation of nearly 3,000 new tracked debris elements. Currently 22,000 of these space debris elements are being actively tracked by U.S. surveillance networks. Each of these debris elements are capable of creating major 
new debris, especially if they collided with another satellite or upper stage rocket. In short, without further remedial action to remove space debris from Earth orbit, the problem will continue to get worse [NASA Office of Orbital Debris] (Pelton, 2015a). Pelton in his interesting book "New Solutions for the Space Debris Problem" discusses with extreme clarity the serious problem of why space services are now of vital importance to our global society. He writes: "Space systems have become so very vital, that if we were suddenly denied access to our space-based infrastructure for weather forecasting and warning, for space-based navigation and timing, for civil and military communications, and for remote sensing and surveillance from space we would be in danger. We would suffer almost immediately-economically, militarily, and socially. Many of our transportation and our communications systems would go down along with our weather and rescue services and defense systems. Internet would lost its synchronization, credit card validation would no longer work, we would not be alerted to major storm systems, air traffic control, shipping navigation, and trucking routing services would be lost.

Unfortunately as our space-based systems have become more and more common, other factors have served to make our satellites more at risk. One risk is that of extreme solar flares and coronal mass ejections."

Then, as mentioned in the introduction, we have a further demonstration of the interconnection of all human activities closely related to each other (Luisi \& Capra, 2014).

In conclusion I can affirm that Humans are so intelligent that manage to contaminate even the space, which is the only escape from the planet!

The risk is that someone in the future will look at our species as we look at that of the dinosaurs!

Then, if we look for "Intelligent Lives" in the Universe .... Why we should look for those like ours? (Giovannelli, 2001b).

However, positive messages can be taken into account: many people are involved in the tentative of protection of the planet from different points of view.

Since the problem of space debris is now urgent, many attempts of removing it are in progress. This imply also legal problems as clearly discussed by Joyeeta Chatterjee (2013) in her thesis on Legal Aspects of Space Debris Remediation: Active Removal of Debris and On-Orbit satellite Servicing, for obtaining the Master Degree in Law.

In this regard it is important to mention the fundamental Handbook of Cosmic Hazards and Planetary Defense (Pelton \& Allahdadi, 2015) that analyzes all the cosmic hazards, including also the pollution produced by humanity in the close space surrounding the Earth, and possible defense. It has been presented in a wonderful way by Lord Martin Rees - Royal Astronomer of the United Kingdom - in his foreword: .... This Handbook is hugely impressive and deserves wide readership: it offers us a broad and well-informed perspective on our cosmic environment. More important, I hope it will stimulate appropriate efforts to understand and counter these threats - an enterprise valuable in its own right, but one which offers an opportunity for international partnership, both governmental and private, in a common cause. We are all members of the crew of "Spaceship Earth" - a precious and fragile "pale blue dot" in the wider cosmos. The recipes and insights in this Handbook will help to protect and safeguard it for future generations (Rees, 2015).

Part XV of this handbook deals with Mounting Hazards of Man-Made Threats in Space (Klinkrad, 2015; Jonas, 2015; Pelton, 2015b; Jonas \& Allahdadi, 2015; Botts, 2015). 
Part XVI of this handbook deals with Future of Planetary Defense (Chatterjee, Pelton \& Allahdadi, 2015; Lubin \& Hughes, 2015; Hertzfeld \& Schieb, 2015; Pelton, 2015c; Tronchetti, 2015; Potter, 2015; Simpson, 2015; Jakhu, 2015; Ross, 2015).

\section{Conclusions}

As I discussed in the introduction, I can state that the Universe is interconnected in all its components: from cosmic network, to clusters of galaxies, to galaxies, to stars, to planets, to living beings, up to the simple bacterium. Therefore every manifestation of life on our planet is subject to interconnection with all the surrounding environment. I can affirm that the whole Universe is a vital whole interconnected with more or less strong links between the various components, but that certainly exist.

I have discussed about the accretion processes in cosmic sources of different nature. We have learned that the accretion is universal as shown in the cartoon of Fig. 47 (Jaime Sanchez Calleja, 2016).

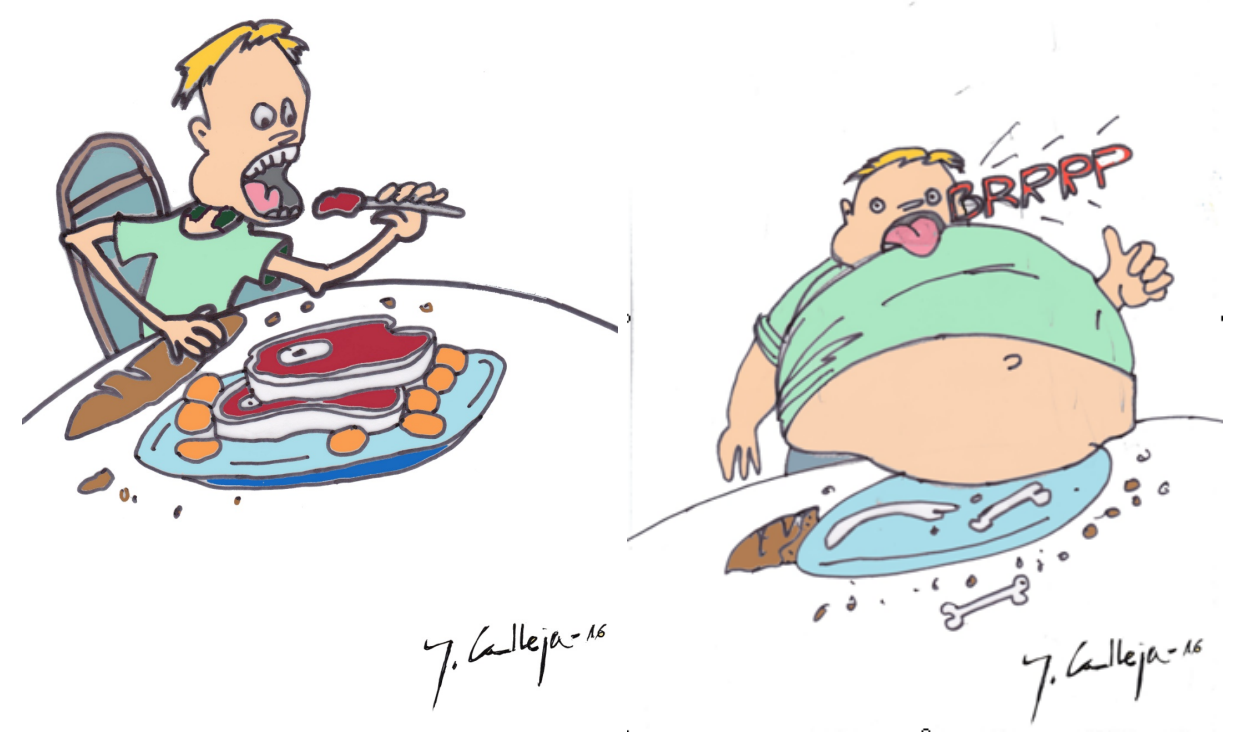

Figure 47: Left panel: Mass accretion onto a thin guy. Right panel: The fat guy after the accretion process (Jaime Sanchez Calleja, 2016).

Finally I can conclude with Fig. 48 that clearly explain all the mysteries of our Universe (Giovannelli, 2000). People who are able to read this sentence can understand that "The truth is written in the book of the Nature. We must learn to read this book".

The experiments provide the basic alphabet, immersed in an apparently chaotic soup, but necessary to understand the nature. From that soup we must extract words and phrases to compose the book of the nature. In other words, the data coming from the experiments constitute the basic alphabet that we use for constructing models that attempt to describe the nature. But we have a lot of models for interpreting the experimental data by the light of science. Depending on the 


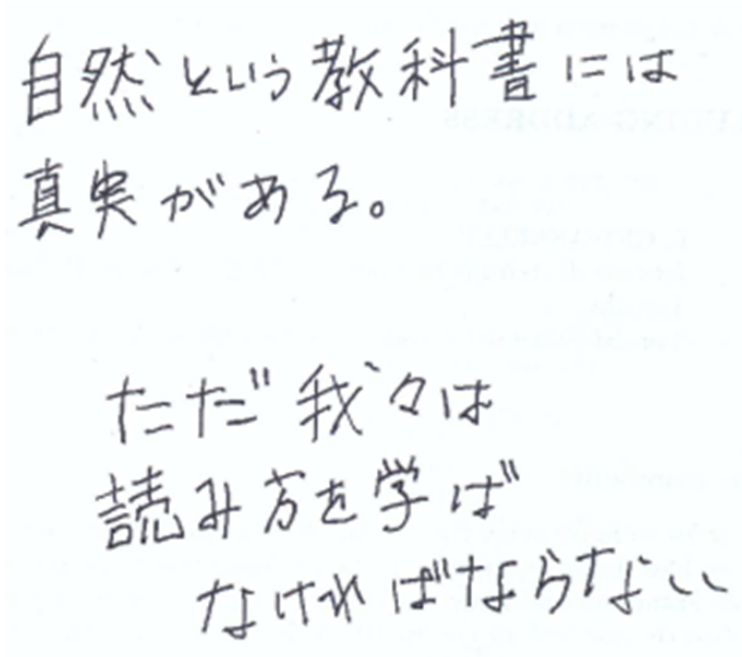

Figure 48: Understanding our Universe (Giovannelli, 2000).

hypotheses the results could run against the experiments. Then, in order to be acceptable, models can take into account and justify $A \mathbf{L L}$ the available data.

The same concept was expressed in much more incisive terms by Richard Phillips Feynman - Nobel laureate in Physics in 1965 - also known as The Great Explainer: It doesn't matter how beautiful your theory is, it doesn't matter how smart you are. If it doesn't agree with experiment, it's wrong.

Acknowledgments This research has made use of:

- The NASA's Astrophysics Data System;

- the NASA Exoplanet Archive, which is operated by the California Institute of Technology, under contract with the National Aeronautics and Space Administration under the Exoplanet Exploration Program;

- the Exoplanet Orbit Database and the Exoplanet Data Explorer at exoplanets.org.

\section{References}

[1] Abbott, B.P., Abbott, R., Abbott, T.D., Abernathy, M.R., Acernese, F. et al.: 2016a, PhRvL 116, Issue 24, id. 241102.

[2] Abbott, B.P., Abbott, R., Abbott, T.D., Abernathy, M.R., Acernese, F. et al.: 2016b, PhRvL 116, Issue 24, id. 241103.

[3] Abbott, B.P., Abbott, R., Abbott, T.D., Abernathy, M.R., Acernese, F. et al.: 2016c, Living Rev. Relativity 19, Issue 1, article id. 1, 39 pp.

[4] Abbott, B.P., Abbott, R., Abbott, T.D., Acernese, F., Ackley, K. et al.: 2017a, PhRvL 119, Issue 16, id.161101. 
[5] Abbott, B.P., Abbott, R., Abbott, T.D., Acernese, F., Ackley, K. et al.: 2017b, ApJL 848, Issue 2, article id. L12, 59 pp.

[6] Abramowicz, M.A., Fragile, P.C.: 2013, Foundations of Black Hole Accretion Disk Theory, Living Rev. Relativity 16, 1-88.

[7] Adams, F.C., Shu, F.H.: 1986, ApJ 308, 836-853.

[8] Adams, F.C., Lada, C.J., Shu, F.H.: 1987, ApJ 312, 788-806.

[9] Adams, F.C., Lada, C.J., Shu, F.H.: 1988, ApJ 326, 865-883.

[10] Adams, F.C., Emerson, J.P., Fuller, G.A.: 1990, ApJ 357, 606-620.

[11] ALMA Partnership; Brogan, C.L., Pérez, L.M., Hunter, T.R., Dent, W.R.F., Hales, A.S. et al.: 2015, ApJ 808, Issue 1, article id. L3, 10 pp.

[12] ALMA Science Advisory Committee: 2015, Major Science Themes in the 2020-2030 Decade, https://science.nrao.edu/facilities/alma/alma-develop-old-022217/ScienceThemes.pdf

[13] Andrè, P., 1994: in The Cold Universe, Montmerle Th., Lada C.J., Mirabel, I.F. \& Trân Thanh Vân, J. (Eds), Editions Frontières, Paris, p. 179.

[14] Andrews, S.M., Wilner, D.J., Zhu, Z., Birnstiel, T., Carpenter, J.M. et al.: 2016, ApJ 820, Issue 2, article id. L40, 5 pp.

[15] Anglada-Escudé, G., Amado, P.J., Barnes, J., Berdiñas, Z.M., Butler, R.P. et al.: 2016, Nature 536, Issue 7617, 437-440.

[16] Ansdell, M., Williams, J.P., van der Marel, N., Carpenter, J.M., Guidi, G. et al.: 2016, ApJ 828, Issue 1 , article id. 46, $15 \mathrm{pp}$.

[17] Antoniucci, S.: 2018, talk at the Saint Petersburg Workshop on Accretion Processes in Cosmic Sources - II.

[18] Antoniucci, S., Alcalá, J.M., Codella, C., Nisini, B. (Eds.): 2015, Disks, Jets and the dawn of planets, eprint arXiv:1506.07073.

[19] Arquilla, R., Goldsmith, P.F.: 1986, ApJ 303, 356-374.

[20] Aso, Y., Ohashi, N., Saigo, K., Koyamatsu, S., Aikawa, Y. et al.: ApJ 812, Issue 1, article id. 27, 20 pp.

[21] Astor, M.: 2017, online at https://www.nytimes.com/2017/09/19/us/hurricanes-irma-harvey-maria.html

[22] Bambi, C. (Ed.): 2016, Astrophysics of Black Holes: From Fundamental Aspects to Latest Developments, Astrophysics and Space Science Library, Volume 440, 207 pp.

[23] Bambi, C.: 2017a, Black Holes: A Laboratory for Testing Strong Gravity, Springer Nature Singapore Pte Ltd., ISBN 978-981-10-4523-3, 340 pp.

[24] Bambi, C.: 2017b, Testing black hole candidates with electromagnetic radiation, PhRvD 95, Issue 10, id. 104035 .

[25] Bambi, C.: 2017c, Astrophysical Black Holes: A Compact Pedagogical Review, arXiv 171110256.

[26] Bath, G.T.: 1969, ApJ 158, 571.

[27] Bath, G.T.: 1975, MNRAS 171, 311. 
[28] Bath, G.T.: 1976, in Structure and Evolution of Close Binary Systems, P. Eggleton, S. Mitton \& J. Whelan (Eds.), IAU Symp. No. 73, p. 173.

[29] Bath, G.T.: 1978, QJRAS 19, 442.

[30] Bath, G.T.: 1980, in Close binary stars: Observations and interpretation, IAU Symp. No. 88, p. 155.

[31] Bath, G.T.: 1984, Ap\&SS 99, 127.

[32] Bath, G.T., Evans, W.D., Papaloizou, J., Pringle, J.E.: 1974, MNRAS 169, 447.

[33] Beall. J.H., Knight, F.K., Smith, H.A., Wood, K.S., Lebofsky, M., Rieke, G.: 1984, ApJ 284, 745-750.

[34] Beckwith, S.V.W.: 1998, talk at the The Physics of Star Formation \& Early Stellar Evolution, NATO/ASI, Crete II, 3 June.

[35] Beckwith, S.V.W.: 1999, in The Origin of Stars and Planetary Systems, Charles J. Lada \& Nikolaos D. Kylafis (Eds.), Kluwer Academic Publishers, p. 579.

[36] Beckwith, S.V.W., Sargent, A.I., Chini, R.S., Güsten, R.: 1990, AJ 99, 924-945.

[37] Beckwith, S.V.W., Sargent, A.I.: 1991, ApJ 381, 250-258.

[38] Beckwith, S.V.W., Sargent, A.I.: 1996, Nature 383, 139-144.

[39] Belczynski, K., Ziółkowski, J.: 2009, ApJ 707, Issue 2, 870-877.

[40] Belczynski, K., Bulik, T., Fryer, C.L., Ruiter, A., Valsecchi, F. et al.: 2010, ApJ 714, Issue 2, 1217-1226.

[41] Beskin, V.S., Balogh, A., Falanga, M., Lyutikov, M., Mereghetti, S., Piran, T., Treumann, R.A. (Eds.): 2015, The Strongest Magnetic Fields in the Universe, Space Sciences Series of ISSI, Vol. 54, Springer Science+Business Media New York, ISBN 978-1-4939-3549-9, 579 pp.

[42] Birnstiel, T.: 2010, The Evolution of Gas and Dust in Protoplanetary Accretion Disks, PhD Thesis, the Ruperto-Carola University of Heidelberg, Germany. arXiv:1107.3466v1 [astro-ph.EP] 18 Jul 2011.

[43] Bisikalo, D.V., Zhilkin, A.G.: 2012, in From Interacting Binaries to Exoplanets: Essential Modeling Tools, M.T. Richards \& I. Hubeny (Eds.), IAU Symp. 282, 509-516.

[44] Bisikalo, D.V., Kononov, D.A., Kaigorodov, P.V., Zhilkin, A.G., Boyarchuk, A.A.: 2008, ARep 52, 318-326.

[45] Bisikalo, D. V., Zhilkin, A.G.: 2015, Acta Pol. CTU Proc. 2,(1), 60-65.

[46] Bisikalo, D.V., Kurbatov, E.P., Pavlyuchenkov, Ya.N., Zhilkin, A.G., Kaygorodov, P.V.: 2016, MNRAS 458, 3892-3903.

[47] Bisnovatyi-Kogan, G.S.: 2011, Stellar Physics: 2: Stellar Evolution and Stability, Astronomy and Astrophysics Library, ISBN 978-3-642-14733-3. Springer-Verlag Berlin Heidelberg.

[48] Bisnovatyi-Kogan, G.S., Giovannelli, F.: 2017, A\&A 599, id. A55, 7 pp.

[49] Blaes, O.: 2014, SSR 183, 21-41.

[50] Blumenthal, G.R., Tucker, W.H.: 1974, Ann. Rev. A\&A 12, 23-46.

[51] Boller, T.: 2017, talk at the Frascati Workshop 2017 Multifrequency Behaviour of High Energy Cosmic Source - XII, Palermo, Italy, 12-17 June.

[52] Boneva, D., Kaigorodov, P.V., Bisikalo, D.V., Kononov, D.A.: 2009, ARep 53, 1004-1012. 
[53] Bonfils, X., Delfosse, X., Udry, S., Forveille, T., Mayor, M. et al.: 2013. A\&A 549, id.A109, 75 pp.

[54] Botts, C.: 2015, in Handbook of Cosmic Hazards and Planetary Defense, Joseph, N. Pelton \& Firooz Allahdadi (Eds.), Springer Reference, (c) Springer International Publishing Switzerland 2015, pp. 891-918.

[55] Bovaird, T., Lineweaver, C.H.: 2013, MNRAS 435, Issue 2, 1126-1138.

[56] Brogan, C.L., Pérez, L. M., Hunter, T.R., Dent, W.R.F., Hales, A.S. et al. (ALMA Partnership): 2015, ApJ 808, Issue 1, article id. L3, 10 pp.

[57] Bruno Giordano Nolano: 1584, De l'infinito, universo et mondi, Stampato in Venezia, Anno MDLXXXIV, in Dialoghi filosofici italiani, a cura di Michele Ciliberto, Mondadori, Milano (2000).

[58] Buckley, D., 2015, talk at the Palermo Workshop on "The Golden Age of Cataclysmic Variables and Related Objects - III", Palermo, Italy, 7-12 September.

[59] Burdanov, A., Delrez, L., Gillon, M., Jehin, E.: 2018, SPECULOOS Exoplanet Search and Its Prototype on TRAPPIST, Springer International Publishing AG, part of Springer Nature.

[60] Burrows, C.J., Stapelfeldt, K.R., Watson, A.M., Krist, J.E., Ballester, G.E. et al.: 1996, ApJ 473, 437-451.

[61] Calvet, N., Hartmann, L., Strom, S.E.: 2000, in Protostars and Planets IV, Mannings, V., Boss, A.P., Russell, S.S. (Eds.), (Book - Tucson: University of Arizona Press), p. 377.

[62] Casares, J.: 2015, ApJ 808, Issue 1, article id. 80, 11 pp.

[63] Casares, J., Jonker, P.G.: 2014, SSRv 183, 223-252.

[64] Casares, J., Negueruela, I., Ribó, M., Ribas, I., Paredes, J.M. et al.: 2014, Nature 505, Issue 7483, 378-381.

[65] Caselli, P.: 2002, Planetary and Space Science 50, 1133-1144.

[66] Caselli, P.: 2003, Ap\&SS 285, 619-631.

[67] Carpenter, J.M., Bouwman, J., Mamajek, E.E., Meyer, M.R., Hillenbrand, L.A. et al.: 2009, ApJS $181,197-226$.

[68] Carpenter, J., Iono, D., Testi, L., Whyborn, N., Wootten, A., Evans, N. (The ALMA Development Working Group): 2018, The Alma Development Roadmap, https://www.almaobservatory.org/en/publications/the-alma-development-roadmap/

[69] Casares, J., Negueruela, I., Ribó, M., Ribas, I., Paredes, J.M. et al.: 2014, Nature 505, Issue 7483, 378-381.

[70] Chatterjee, J.: 2013, Legal Aspects of Space Debris Remediation: Active Removal of Debris and On-Orbit satellite Servicing, Master of Law Degree Thesis, McGill University, Montreal, Canada. Online at http://digitool.library.mcgill.ca/webclient/StreamGate?folder ${ }_{i} d=0 \& d v s=1552495872672 \sim 456$

[71] Chatterjee, J., Pelton, J.N., Allahdadi, F.: 2015, in Handbook of Cosmic Hazards and Planetary Defense, Joseph, N. Pelton \& Firooz Allahdadi (Eds.), Springer Reference, (c) Springer International Publishing Switzerland 2015, pp. 921-940.

[72] Chaty, S.: 2011, in Evolution of Compact Binaries, L. Schmidtobreick, M. Schreiber \& C. Tappert (Eds.), ASP Conf. Ser., 447, 29-43.

[73] Chiang, E.I., Goldreich, P.: 1997, ApJ 490, 368-376. 
[74] Cieza, L.A., Casassus, S., Tobin, J., Bos, S.P., Williams, J.P. et al.: 2016, Nature 535, Issue 7611, 258-261.

[75] Cleeves, L.I., Bergin, E.A., Alexander, C.M. O.'D., Du, F., Graninger, D. et al.: 2014, Science, 345, Issue 6204, 1590-1593.

[76] Cohen, M.: 1983, ApJ 270, L69-L71.

[77] Coleiro, A., Chaty, S.: 2013, ApJ 764, Issue 2, article id. 185, 14 pp.

[78] Contopoulos, I., Gabuzda, D., Kylafis, N. (Eds.): 2015, The Formation and Disruption of Black Hole Jets, Astrophysics and Space Science Library, Volume 414. ISBN 978-3-319-10355-6. Springer International Publishing Switzerland, 273 pp.

[79] Cooley, J.W., Tukey, J.W.: 1965, An algorithm for the machine calculation of complex Fourier series, Math. Comput. 19, 297-301.

[80] Cottin, H., Kotler, J.M., Bartik, K., Cleaves, H.J., Cockell, C.S., de Vera, J.-P.P. etal.: 2017a, SSRv $209,1-42$.

[81] Cottin, H., Kotler, J.M., Billi, D., Cockell, C., Demets, R. Ehrenfreund, P. et al.: 2017b, SSRv 209, 83-181.

[82] Dallilar, Y., Eikenberry, S.S., Garner, A., Stelter, R.D., Gottlieb, A. et al.: 2017, Science 358, Issue 6368, 1299-1302.

[83] Delrez, L., Gillon, M., Queloz, D., Demory, B.-O., Almleaky, Y.: 2018, SPIE 10700, id. 107001I, 21 pp.

[84] Dent, W.R.F., Wyatt, M.C., Roberge, A., Augereau, J.-C., Casassus, S. et al.: 2014, Science 343, Issue 6178, 1490-1492.

[85] Dong, R., Zhu, Z., Whitney, B.: 2015, ApJ 809, Issue 1, article id. 93, 18 pp.

[86] Drake, F.D.: 1962, Intelligent Life in Space, New York: Macmillan, 128 pp.

[87] Drouart, A., Dubrulle, B., Gautier, D., Robert, F.: 1999, Icarus 140, 129-155.

[88] Dullemond, C.P., Hollenbach, D., Kamp, I., D’Alessio, P.: 2007, in Protostars and Planets, V,B. Reipurth, D. Jewitt \& K. Keil (Eds.), University of Arizona Press, Tucson, 951 pp., p. 555-572.

[89] Dullemond, C.P., Monnier, J.D.: 2010, ARA\&A 48, 205-239.

[90] Dutrey, A., Guilloteau, S., Duvert, G., Prato, L., Simon, M. Schuster, K., Menard, F.: 1996, A\&A 309, 493-504.

[91] Dutrey, A., Guilloteau, S., Ho, P.: 2007, in Protostars and Planets V, B. Reipurth, D. Jewitt \& K. Keil (Eds.), 951 pp., University of Arizona Press, Tucson, p. 495-506.

[92] Dyson, F.: 1960, Science, 131, Issue 3414, 1667-1668.

[93] Elsasser, H., Staude, H.J.: 178, A\&A 70, L3-L6.

[94] Enoch, M.L., Corder, S., Dunham, M.M., Duchêne, G.: 2009, ApJ 707, 103-113.

[95] Falanga, M., Belloni, T., Casella, P., Gilfanov, M., Jonker, P., King, A. (Eds.): 2015, The Physics of Accretion onto Black Holes, Springer, Space Sciences Series of ISSI, Vol. 49, 483 pp.

[96] Fang, M., Pascucci, I., Edwards, S., Gorti, U., Banzatti, A. et al.: 2018, ApJ 868, Issue 1, article id. 28, 35 pp. 
[97] Feigelson, E.D., Montmerle, T.: 1999, High-energy processes in young stellar objects, Annu. Rev. Astron. Astrophys. 37, 363-408.

[98] Ferrario, L., de Martino, D., Gänsicke, B.T.: 2015, SSRv 191, 111-169.

[99] Fischer, A., Beuermann, K.: 2001, A\&A 373, 211-221.

[100] Fisher-Quann, R.: 2017, Is Space Junk a problem?, Quark Magazine. Online at https://quarkmag.co $\mathrm{m} /$ is-space-junk-a-problem-67e865f702a9.

[101] Frank, J., King, A.R., Raine, D.J.: 1985, Accretion power in astrophysics, Cambridge and New York, Cambridge University Press, 283 pp.

[102] Frank, J., King, A., Raine, D.J.: 2002, Accretion Power in Astrophysics - Third Edition, Cambridge, UK: Cambridge University Press, pp. 398. ISBN 0521620538.

[103] Fryer, C.L., Belczynski, K., Wiktorowicz, G., Dominik, M., Kalogera, V., Holz, D.E.: 2012, ApJ 749, Issue 1, article id. 91, 14 pp.

[104] Gauguin, P.: 1897, Oil Painting preserved in the Museum of Fine Arts in Boston, Ma, USA.

[105] Geyer, R., Jambeck, J.R., Law, K.L.: 2017, Sci. Adv. 3, e1700782, 5 pp.

[106] Giovannelli, F.: 1994, SSRv 69, 1-138.

[107] Giovannelli, F.: 2000, in The Evolution of The Milky Way, F. Matteucci \& F. Giovannelli (Eds.), Kluwer Academic Publishers, pp. 619-620.

[108] Giovannelli, F.: 2001a, The Bridge between the Big Bang and Biology (Stars, Planetary Systems, Atmospheres, Volcanoes: Their Link to Life), F. Giovannelli (ed.), President Bureau of the CNR, Roma, Italy, 440 pp.

[109] Giovannelli, F.: 2001b, in The Bridge between the Big Bang and Biology (Stars, Planetary Systems, Atmospheres, Volcanoes: Their Link to Life), F. Giovannelli (ed.), President Bureau of the CNR, Roma, Italy, p. 439.

[110] Giovannelli, F.: 2016, in The 4th Annual Conference on High Energy Astrophysics in Southern Africa (HEASA 2016), Online at http://pos.sissa.it/cgi-bin/reader/conf.cgi?confid=275, id.31.

[111] Giovannelli, F., Karakuła, S., Tkaczyk, W.: 1981, in Origin of Cosmic Rays, Setti, G., Spada, G. \& Wolfendale, A.W. (Eds.), IAU Symp. 94, p. 335-336.

[112] Giovannelli, F., Karakuła, S., Tkaczyk, W.: 1982, AcA 32, no. 1-2, 121-130.

[113] Giovannelli, F., Ziółkowski, J.: 1990, AcA 40, 95-103.

[114] Giovannelli, F., Vittone, A.A., Rossi, C., Errico, L., Bisnovatyi-Kogan, G.S., Kurt, V.G., Lamzin, S.A. et al.: 1995, A\&AS 114, 341.

[115] Giovannelli, F., Sabau-Graziati, L.: 2001, Ap\&SS, 276, 67-80.

[116] Giovannelli, F., Sabau-Graziati, L.: 2004, SSR, 112, 1-443.

[117] Giovannelli, F., Sabau-Graziati, L. (Eds.): 2008, Multifrequency Behaviour of High Energy Cosmic Sources - VII, Ch. J. A\&A, Supplement, Vol. 8, 426 pp.

[118] Giovannelli, F., Sabau-Graziati, L.: 2010. talk at the Palermo Workshop on Science with MILLIMETRON.

[119] Giovannelli, F., Sabau-Graziati, L.: 2011, Acta Polytechnica 51, No. 2, 21-32. 
[120] Giovannelli, F., Sabau-Graziati, L.: 2012a, in The Golden Age of Cataclysmic Variables and Related Objects, F. Giovannelli \& L. Sabau-Graziati (eds.), Mem. S.A.It., 83 N. 2, 698-707.

[121] Giovannelli, F., Sabau-Graziati, L. (Eds.): 2012b, The Golden Age of Cataclysmic Variables and Related Objects - I, Mem. SAIt. 83, 433 pp.

[122] Giovannelli, F., Bisnovatyi-Kogan, G.S., Klepnev, A.S.: 2013, A\&A 560, id.A1, 11 pp (GBK13).

[123] Giovannelli, F., Sabau-Graziati, L.: 2014a, in Multifrequency Behaviour of High Energy Cosmic Sources - X, F. Giovannelli \& L. Sabau-Graziati (Eds.), Acta Polytechnica, CTU Proc. ISSN 978-80-01-05668-4 e-ISSN 2336-5382, Vol. 1 No. 1, 1-12.

[124] Giovannelli, F., Sabau-Graziati, L. (Eds.): 2014b, Multifrequency Behaviour of High Energy Cosmic Sources - X, Acta Polytechnica, CTU Proc. ISSN 978-80-01-05668-4 e-ISSN 2336-5382, Vol. 1 No. $1,331 \mathrm{pp}$.

[125] Giovannelli, F., Bisnovatyi-Kogan, G.S., Bruni, I., Corfini, G., Martinelli, F., Rossi, C.: 2015, AcA $65,107-116$.

[126] Giovannelli, F., Sabau-Graziati, L.: 2015a, The Golden Age of Cataclysmic Variables and Related Objects - III, Online at http://pos.sissa.it/cgi-bin/reader/conf.cgi?confid=255, id. 1, pp. 1-36.

[127] Giovannelli, F., Sabau-Graziati, L. (Eds.): 2015b, The Golden Age of Cataclysmic Variables and Related Objects - III, Online at http://pos.sissa.it/cgi-bin/reader/conf.cgi?confid=255

[128] Giovannelli, F., Sabau-Graziati, L. (Eds.): 2015c, The Golden Age of Cataclysmic Variables and Related Objects - II, Acta Polytechnica, CTU Proc. Vol. 2, No. 1, 333 pp.

[129] Giovannelli, F., Sabau-Graziati, L.: 2016, in Accretion Processes in Cosmic Sources, F. Giovannelli (Ed.), online at http://pos.sissa.it/cgi-bin/reader/conf.cgi?confid=288, id.1, pp. 1-45.

[130] Giovannelli, F., Sabau-Graziati, L. (Eds.): 2017, The Golden Age of Cataclysmic Variables and Related Objects - IV, Online at http://pos.sissa.it/cgi-bin/reader/conf.cgi?confid=315.

[131] Giovannelli, F., Sabau-Graziati, L.: 2018, in Frontier Research in Astrophysics - III, https://pos.sissa.it/cgi-bin/chairman/chlist.cgi?confid=331, in press.

[132] Goliasch, J., Nelson, L.: 2015, ApJ 809, Issue 1, article id. 80, 19 pp.

[133] González-Casanova, D.F., Lazarian, A., Santos-Lima, R.: 2016, ApJ 819, Issue 2, article id. 96,11 pp.

[134] Goodman, J.: 1993, ApJ 406, 596-613.

[135] Gorti, U., Liseau, R., Sándor, Z., Clarke, C.: 2016, SSRv 205, 125-152.

[136] Grimm, H.-J.: 2003, PhD Thesis, Ludwig-Maximilians-Universitat, München, Germany.

[137] Grudzinska, M., Belczynski, K., Casares, J., de Mink, S.E., Ziolkowski, J. et al.: 2015, MNRAS, 452, Issue 3, 2773-2787.

[138] Han, E., Wang, S., Wright, J.T., Feng, Y.K., Zhao, M. et al.: 2014, PASP, 126, 827-837.

[139] Hartmann, L.: 1998, Accretion Processes in Star Formation, Cambridge, UK; New York: Cambridge University Press, (Cambridge astrophysics series; 32). ISBN 0521435072.

[140] Hartmann, L., Kenyon, S.J.: 1987a, ApJ 312, 243-253.

[141] Hartmann, L., Kenyon, S.J.: 1987b, ApJ 322, 393-398. 
[142] Hartwig, T., Volonteri, M., Bromm, V., Klessen, R.S., Barausse, E. et al.: 2016, MNRAS Lett. 460, Issue 1, L74-L78.

[143] Henning, Th., Michel, B., Stognienko, R.: 1995, P\&SS 43, 1333-1343.

[144] Hertzfeld, H.R., Schieb, P.-A.: 2015, in Handbook of Cosmic Hazards and Planetary Defense, Joseph, N. Pelton \& Firooz Allahdadi (Eds.), Springer Reference, (c) Springer International Publishing Switzerland 2015, pp. 993-1006.

[145] van den Heuvel, E.P.J.: 2009, in Physics of Relativistic Objects in Compact Binaries: From Birth to Coalescence, Ap\&SS Library, 359, 125.

[146] Horne, K.: 1985, MNRAS 213, 129-141.

[147] Illarionov, A.F., Sunyaev, R.A.: 1975, A\&A 39, 185-195.

[148] Hillenbrand, L.A.: 2008, Phys. Scr. T130, id. 014024, 7 pp.

[149] Isella, A.: 2006, PhD Thesis, University of Milano, Italy.

[150] Isella, A.: 2016, in From Interstellar Clouds to Star-Forming Galaxies: Universal Processes?, IAU Symp. 315, 107-113.

[151] Isella, A., Carpenter, J.M., Sargent, A.I.: 2009, ApJ 701, Issue 1, 260-282.

[152] Isella, A., Turner, N.: 2016, arXiv:1608.05123.

[153] Israel, G.: 1996, Ph.D. thesis, Scuola Int. Superiore Stud. Avanzati (SISSA), Trieste, http://www.mporzio.astro.it/gianluca/phdthesis.html

[154] Jakhu, R.S.: 2015, in Handbook of Cosmic Hazards and Planetary Defense, Joseph, N. Pelton \& Firooz Allahdadi (Eds.), Springer Reference, (c) Springer International Publishing Switzerland 2015, pp. 1069-1084.

[155] Jeffers, S.V., Min, M., Canovas, H., Rodenhuis, M., Keller, C.U.: 2014, A\&A 561, id. A23, 9 pp.

[156] Jehin, E., Gillon, M., Queloz, D., Magain, P., Manfroid, J. et al.: 2011, Msngr 145, 2-6.

[157] Jehin, E., Gillon, M., Queloz, D., Delrez, L., Burdanov, A. et al.: 2018, Msngr 174, 2-7.

[158] Johnston, R.: 2004, http://www.johnstonsarchive.net/relativity/bhctable.html

[159] Jonas, F.M.: 2015, in Handbook of Cosmic Hazards and Planetary Defense, Joseph, N. Pelton \& Firooz Allahdadi (Eds.), Springer Reference, (c) Springer International Publishing Switzerland 2015, pp. 835-849.

[160] Jonas, F.M., Allahdadi, F.:: 2015, in Handbook of Cosmic Hazards and Planetary Defense, Joseph, N. Pelton \& Firooz Allahdadi (Eds.), Springer Reference, (c) Springer International Publishing Switzerland 2015, pp. 875-889.

[161] Kardashev, N.S., Novikov, I.D., Lukash, V.N., Pilipenko, S.V., Mikheeva, E.V. et al.: 2014, Physics-Uspekhi Vol. 57, Issue 12, article id. 1199-1228.

[162] Kastner, J.H., Qi, C., Dickson-Vandervelde, D.A., Hily-Blant, P., Forveille, T. et al.: 2018. ApJ 863, Issue 1, article id. 106, 14 pp.

[163] Kataoka, A., Tsukagoshi, T., Momose, M., Nagai, H., Muto, T. et al.: 2016a, ApJ 831, Issue 2, article id. L12, 6 pp.

[164] Kataoka, A., Muto, T., Momose, M., Tsukagoshi, T., Dullemond, C.P.: 2016b, ApJ 820, Issue 1, article id. 54, 8 pp. 
[165] King, A.: 2018, talk at Saas-Fee on Black Hole Accretion and Feedback, online at https://indico.cern.ch/event/626266/contributions/2807660/attachments/1593311/2522405/saas-feeunderscore-king-underscore-1.pdf

[166] Kitamoto, S., Enoto, T., Safi-Harb, S., Pottschmidt, K., Ferrigno, C. et al.: 2014, arXiv:1412.1165v1 [astro-ph.HE] 3 Dec 2014.

[167] Klinkrad, H.: 2015, in Handbook of Cosmic Hazards and Planetary Defense, Joseph, N. Pelton \& Firooz Allahdadi (Eds.), Springer Reference, (c) Springer International Publishing Switzerland 2015, pp. 807-834.

[168] Kononov, D.A., Giovannelli, F., Bruni, I., Bisikalo, D.V.: 2012, A\&A 538, id. A94, 7 pp.

[169] Kormendy, J., Ho, L.C.: 2013, Ann. Rev. A\&A 51, 511-653.

[170] Kotze, E.J., Potter, S.B., McBride, V.A.: 2015, A\&A 579, id. A77, 9 pp.

[171] Kotze, E.J., Potter, S.B., McBride, V.A.: 2016, A\&A 595, id. A47, 12 pp.

[172] Kratter, K., Lodato, G.: 2016, Annu. Rev. Astron. Astrophys. 54, 271-311.

[173] Lamzin, S.A., Bisnovatyi-Kogan, G.S., Errico, L., Giovannelli, F., Katysheva, N.A., Rossi, C., Vittone, A.A.: 1996, A\&A 306, 877.

[174] Lasota, J.-P.: 2001, New Astron. Rev. 45, 449-508.

[175] Lasota, J.-P.: 2016, Black Hole Accretion Discs, in Astrophysics of Black Holes, Cosimo Bambi (Ed.), Astrophysics and Space Science Library, Volume 440. ISBN 978-3-662-52857-0. Springer-Verlag Berlin Heidelberg, p. 1.

[176] Lebreton, L., Slat, B., Ferrari, F., Sainte-Rose, B., Aitken, J. et al.: 2018, Scientific Reports, 8:4666, DOI:10.1038/s41598-018-22939-w, www.nature.com/scientificreports, pp. 1-15.

[177] Lewin, W.H.G., van den Heuvel, E.P.J. (Eds.): 1983, Accretion-Driven Stellar X-ray Sources, Cambridge University Press, 450 pp.

[178] LIGO-VIRGO Gravitational Wave Open Science Center: 2018, Online at https://www.gw-openscience.org/catalog/GWTC-1-confident/html/

[179] Lineweaver, C.H., Fenner, Y. \& Gibson, B.K.: 2004, Science, 303, Issue 5654, 59-62.

[180] Lineweaver, C.H., Chopra, A.: 2012, Ann. Rev. of Earth and Planetary Sci., 40, issue 1, 597-623.

[181] Lipunov, V.M.: 1987, Ap\&SS 132, no. 1, 1-51.

[182] Lipunov, V.M.: 1995, in Frontier Objects in Astrophysics and Particle Physics, F. Giovannelli \& G. Mannocchi (Eds.), SIF, Bologna, Italy, 47, 61-76.

[183] Lipunov, V.M.: 2018, talk at the Saint Petersburg Workshop on Accretion Processes in Cosmic Sources - II, Saint Petersburg (Russian Federation), 3-8 September.

[184] Lipunov, V.M., Postnov, K.A.: 1988, Ap\&SS 145, no. 1, 1-45.

[185] Liu, Q.Z., van Paradijs, J., van den Heuvel, E.P.J.: 2006, A\&A 455, 1165-1168.

[186] Liu, Q.Z., van Paradijs, J., van den Heuvel, E.P.J.: 2007, A\&A 469, 807-810.

[187] Lubin, P, Hughes, G.B.: 2015, in Handbook of Cosmic Hazards and Planetary Defense, Joseph, N. Pelton \& Firooz Allahdadi (Eds.), Springer Reference, (c) Springer International Publishing Switzerland 2015, pp. 941-991. 
[188] Luisi, P.L.: 2018, Talk at the Cortona Friends - Todi Week on Science and Spirituality, Todi, Italy, 22-30 June.

[189] Luisi, P.L., Capra, F.: 2014, The systems View of Life, Cambridge Univ. Press, 510 pp.

[190] Lynden-Bell, D., Pringle, J.E.: 1974, MNRAS 168, 603-637.

[191] Machida, M.N., Inutsuka, S., Matsumoto, T.: 2010, ApJ 724, 1006-1020.

[192] Magorrian, J., Tremaine, S.: 1999, MNRAS 309, 447-460.

[193] Manara, C.F., Rosotti, G., Testi, L., Natta, A., Alcalá, J.M. et al.: 2016, A\&A 591, id. L3, 6 pp.

[194] Mantle, V.J., Bath, G.T.: 1983, MNRAS 202, 151-157.

[195] Marsh, T.R., Horne, K.: 1988, MNRAS 235, 269-286.

[196] Marsh, T.R.: 2001, in Astrotomography, Indirect Imaging Methods in Observational Astronomy, H.M.J. Boffin, D. Steeghs \& J. Cuypers (Eds.), Lecture Notes in Physics 573, 1-27.

[197] de Martino, D., Sala, G., Balman, S., Bernardini, F., Bianchini, A. et al.: 2015, White Paper in Support of the Mission Concept of the Large Observatory for X-ray Timing, arXiv:1501.02767.

[198] Maruyama, S., Ebisuzaki, T., Kurokawa, K.: 2018, talk at the Mondello Workshop on Frontier Research in Astrophysics - II, Palermo, Italy, May 28 - June 2.

[199] McCaughrean, M.J., O’dell, C.R.: 1996, AJ 111, 1977-1987.

[200] McClintock, J.E., Narayan, R., Rybicki, G.B.: 2004, ApJ 615, Issue 1, 402-415.

[201] McConnell, N.J., Ma, C-P., Gebhardt, K., Wright, S.A., Murphy, J.D. et al.: 2011, Nature 480, Issue 7376, 215-218.

[202] Meyer, M.R., Beckwith, S.V.W.: 2000, in ISO Surveys of a Dusty Universe, D. Lemke, M. Stickel \& K. Wilke (Eds.), Lecture Notes in Physics 548, 341.

[203] Meyer, M.R., Backman, D., Beckwith, S.V.W., Brooke, T.Y., Carpenter, J.M. et al.: 2002, in The Origin of Stars and Planets: The VLT View, J.F. Alves \& M.J. McCaughrean (Eds.), ESO ASTROPHYSICS SYMPOSIA. ISBN 3-540-43541-7. Springer-Verlag, 463-470.

[204] Meyer, M.R., Hillenbrand, L.A., Backman, D., Beckwith, S., Bouwman, J. et al.: 2006, PASP 118, 1690-1710.

[205] Meyer, M.R., Backman, D.E., Weinberger, A.J., Wyatt, M.C.: 2007, in Protostars and Planets V, B. Reipurth, D. Jewitt \& K. Keil (Eds.), 951 pp., University of Arizona Press, Tucson, p. 573-588. Also arXiv:astro-ph/0606399.

[206] Michel, F.C.: 1972, Ap\&SS 15, Issue 1, 153-160.

[207] Miotello, A., van Dishoeck, E.F., Kama, M., Bruderer, S.: 2016, A\&A 594, id. A85, 19 pp.

[208] Mudd, D., Martini, P., Zu, Y., Kochanek, C., Peterson, B.M. et al.: 2018, ApJ 862, Issue 2, article id. $123,13 \mathrm{pp}$.

[209] Mundt, R., Fried, J.W.: 1983, ApJ 274, L83-L86.

[210] Nagase, F.: 1989, PASJ 41, no. 1, 1-79.

[211] Natta, A., Grinin, V., Mannings, V.: 2000, in Protostars and Planets IV, Mannings, V., Boss, A.P., Russell, S.S. (Eds.), (Book - Tucson: University of Arizona Press), p. 559-588.

[212] Nelson, L.: 2012, Journal of Physics: Conference Series, Volume 341, Issue 1, id. 012008. 
[213] NOAA, Marine Debris Program: 2012, https://marinedebris.noaa.gov/info/patch.html

[214] O'dell, C.R., Wen, Z.: 1994, ApJ 436, no. 1, 194-202.

[215] Orosz, J.A., Remillard, R.A., Bailyn, C.D., McClintock, J.E.: 1997, ApJL 478, L83-L86.

[216] Paczyński, B.: 1965, AcA 15, 89-102.

[217] Paczyński, B.: 1977, ApJ 216, 822-826.

[218] Pekurovsky, D.: 2012, Siam J. Sci. Comput. Vol. 34, No. 4, pp. C192Û́C209, Society for Industrial and Applied Mathematics.

[219] Pelton, J.N.: 2015a, New Solutions for the Space Debris Problem, Springer Cham Heidelberg New York. Dordrecht London.

[220] Pelton, J.N.: 2015b, in Handbook of Cosmic Hazards and Planetary Defense, Joseph, N. Pelton \& Firooz Allahdadi (Eds.), Springer Reference, (c) Springer International Publishing Switzerland 2015, pp. 851-874.

[221] Pelton, J.N.: 2015c, in Handbook of Cosmic Hazards and Planetary Defense, Joseph, N. Pelton \& Firooz Allahdadi (Eds.), Springer Reference, (C) Springer International Publishing Switzerland 2015, pp. 1007-1025.

[222] Pelton, J.N., Allahdadi, F. (Eds.): 2015, Handbook of Cosmic Hazards and Planetary Defense, Springer Reference, (c) Springer International Publishing Switzerland 2015, 1127 pp.

[223] Pérez, L.M., Carpenter, J.M., Andrews, S.M., Ricci, L., Isella, A. et al.: 2016, Science 353, Issue 6307, 1519-1521.

[224] Potter, M: 2015, in Handbook of Cosmic Hazards and Planetary Defense, Joseph, N. Pelton \& Firooz Allahdadi (Eds.), Springer Reference, (c) Springer International Publishing Switzerland 2015, pp. 1045-1053.

[225] Pringle, J.E.: 1981, Accretion Discs in Astrophysics, ARA\&A 19, 137-162.

[226] Qi, C., Öberg, K.I., Wilner, D.J., Rosenfeld, K.A.: 2013, ApJ 765, Issue 1, article id. L14, 5 pp.

[227] Raguzova, N.V., Lipunov, V.M.: 1999, A\&A 349, 505-510.

[228] Rees, M.J.: 1988a, in Origins, A.C. Fabian (ed.), Cambridge University Press, 1.

[229] Rees, M.J.: 1988b, Nature, 333, 523-528.

[230] Rees, M.J.: 2015, in Handbook of Cosmic Hazards and Planetary Defense, Joseph, N. Pelton \& Firooz Allahdadi (Eds.), Springer Reference, (c) Springer International Publishing Switzerland 2015, p. vii.

[231] Ritter, H., Kolb, U.: 2003, A\&A 404, 301-303.

[232] Rixner, T.A., Siber, T.: 1824, Leben und Lehrmeinungen berühmter Physiker, Sulzbach, Heft 5.

[233] Roberts, L.D.: 1992, Addressing the Problem of Orbital Space Debris: Combining International Regulatory and Liability Regimes, Boston College International \& Comparative Law Review, Vol. XV, No.1, 51-73. Online at http://lawdigitalcommons.bc.edu/iclr/vol15/iss1/4.

[234] Roddier, F.: 1999, in Working on the Fringe: Optical and IR Interferometry from Ground and Space, Stephen Unwin and Robert Stachnik (Eds.), ASPC 194, 318-324.

[235] Roddier, F.: 2004, Adaptive Optics in Astronomy, 419 pp. Cambridge, UK: Cambridge University Press. 
[236] Rodenhuis, M., Sprenger, B., Keller, C.U.: 2012, in Ground-based and Airborne Instrumentation for Astronomy IV., Proc. of the SPIE 8446, article id. 84469J, 6 pp.

[237] Rodriguez-Gil, P.: 2003, Ph.D. Thesis, La Laguna University, Spain.

[238] Ross, S.: 2015, in Handbook of Cosmic Hazards and Planetary Defense, Joseph, N. Pelton \& Firooz Allahdadi (Eds.), Springer Reference, (c) Springer International Publishing Switzerland 2015, pp. 1085-1107.

[239] Rucinski, S.M.: 1985, AJ 90, 2321-2330.

[240] Sakai, N., Sakai, T., Hirota, T., Watanabe, Y., Ceccarelli, C. et al.: 2014, Nature 507, Issue 7490, 78-80.

[241] Salewski, M., Geiger, B., Heidbrink, W.W., Jacobsen, A.S., S B Korsholm, S.B., et al. (the ASDEX Upgrade Team): 2015, Plasma Phys. Control. Fusion 57, 014021, 10 pp.

[242] Sanchez Calleja, J.: 2016, Very funny cartoons produced in occasion of the 2016 Saint Petersburg Workshop on Accretion Processes in Cosmic Sources, Saint Petersburg (Russian Federation, 5-10 September.

[243] Santangelo, G., Murillo, N.M., Nisini, B., Codella, C., Bruderer, S. et al.: 2015, A\&A 581, id.A91, 7 pp.

[244] Saraceno, P.: 2012, Beyond the Stars: Our Origins and the Search for Life in the Universe, World Scientific Publishing Co. Pte. Ltd.

[245] Sargent, A.I., Beckwith, S.: 1987, ApJ 323, 294-305.

[246] Sargent, A.I., Beckwith, S.V.W.: 1989, in Structure and Dynamics of the Interstellar Medium, IAU Colloq. 120, Guillermo Tenorio-Tagle, Mariano Moles, and Jorge Melnick (Eds.), Lecture Notes in Physics 350, 215-220.

[247] Scaringi, S.: 2015, talk at the Palermo Workshop on The Golden Age of CVs and Related Objects III, Palermo, Italy, 7-12 September.

[248] Schmidtobreick, L., Tappert, C.: 2014, in Stella Novae: Past and Future Decades, P.A. Woudt \& V.A.R.M. Ribeiro (Eds.). ASP Conf. Ser. Vol. 490, 29-33.

[249] Schmidtobreick, L., Tappert, C.: 2015, Acta Polytechnica CTU Proc. Vol. 2, 188-191.

[250] Schulze-Makuch, D. \& Davies, P.: 2013, JBIS, 66, 11-14.

[251] Shahbaz, T., Bandyopadhyay, R.M., Charles, P.A., Wagner, R.M., Muhli, P., et al.: 1998, MNRAS 300, 1035-1040.

[252] Shakura, N.I.: 1972, Astron. Zh. 49, 921-929.

[253] Shakura, N.I., Sunyaev, R.A.: 1973, A\&A 24, 337-355.

[254] Shandarin, S., Habib, S., Heitmann, K.: 2010, PhRv D, 81, Issue 10, id. 103006.

[255] Shaviv, G.: 2016, in Accretion Processes in Cosmic Sources, Online at http://pos.sissa.it/cgi-bin/reader/conf.cgi?confid=288, id. 60 .

[256] Simpson, M.K.: 2015, in Handbook of Cosmic Hazards and Planetary Defense, Joseph, N. Pelton \& Firooz Allahdadi (Eds.), Springer Reference, (C) Springer International Publishing Switzerland 2015, pp. 1055-1067.

[257] Smak, J.: 1962, AcA 12, 28-54. 
[258] Smak, J.: 1972, AcA 22, 1-9.

[259] Smak, J.: 1981, AcA 31, 395-408.

[260] Smak, J.: 1984, PASP, 96, 5-18.

[261] Smak, J.: 1985a, in Multifrequency Behaviour of Galactic Accreting Sources, F. Giovannelli (Ed.), SIDEREA, Roma, Italy, pp. 3-16.

[262] Smak, J.: 1985b, in Multifrequency Behaviour of Galactic Accreting Sources, F. Giovannelli (Ed.), SIDEREA, Roma, Italy, pp. 17-36.

[263] Smirnov, A.V., Baryshev, A.M., Pilipenko, S.V., Myshonkova, N.V., Bulanov, V.B. et al.: 2012, SPIE 8442, article id. 84424C, 9 pp.

[264] Stoiko, M.: 1970, Soviet Rocketry: Past. Present, and Future, 272 pp., Publisher: Holt, Rinehart and Winston; 1st edition.

[265] Strom, K.M., Strom, S.E., Edwards, S., Cabrit, S., Skrutskie, M.F.: 1989a, AJ 97, 1451-1470.

[266] Strom, K.M., Newton, G., Strom, S.E., Seaman, R.L., Carrasco, L. et al.: 1989b, ApJS 71, 183-217.

[267] Strong, A.W., Wolfendale, A.W., Worrall, D.M.: 1976, Journal of Physics, Part A - Mathematical and General, vol. 9, 1553-1566.

[268] Tanaka, Y.: 2002, in The Century of Space Science, J.A. Bleeker, J. Geiss \& M. Huber (Eds.), Kluwer Academic Publishers, pp. 839-856.

[269] Tazzari, M., Testi, L., Ercolano, B., Natta, A., Isella, A. et al.: 2016, A\&A 588, id. A53, 19 pp.

[270] Tinetti, G., Drossart, P., Eccleston, P., Hartogh, P., Heske, A. et al.: 2018, ExA 46, Issue 1, 135-209.

[271] Tronchetti, F.: 2015, in Handbook of Cosmic Hazards and Planetary Defense, Joseph, N. Pelton \& Firooz Allahdadi (Eds.), Springer Reference, (c) Springer International Publishing Switzerland 2015, pp. 1027-1043.

[272] Walsh, C., Loomis, R.A., Öberg, K.I., Kama, M., van 't Hoff, M.L.R. et al.: 2016, ApJ 823, Issue 1, article id. L10, 7 pp.

[273] Watson, A.M., Stapelfeldt, K.R., Wood, K., Ménard, F.: 2007, in Protostars and Planets V, B. Reipurth, D. Jewitt \& K. Keil (Eds.), University of Arizona Press, Tucson, p. 523-538.

[274] Wheatley, P.J., Mauche, C.W., Mattei, J.A.: 2003, MNRAS 345, 49-61.

[275] White, R.J., Greene, T.P., Doppmann, G.W., Covey, K.R., Hillenbrand, L.A.: 2007, in Protostars and Planets, V.B. Reipurth, D. Jewitt \& K. Keil (Eds.), University of Arizona Press, Tucson, p. 117-132. Also arXiv:astro-ph/0604081.

[276] Wiktorowicz, G. Belczynski, K., Maccarone, Th.: 2014, arXiv:1312.5924.

[277] Wolf, S., Malbet, F., Alexander, R., Berger, J.-P., Creech-Eakman, M. et al.: 2012, A\&ARv 20, id. $52,83 \mathrm{pp}$.

[278] Wu, K.: 2009, Res. Astron. Astrophys., 9 (Issue 7), 725-744.

[279] Wu, K., Ramsay, G. \& Willes, A.: 2008, Ch. J. A\&A. Vol. 8, Suppl., 169-174.

[280] Wu, Q., Czerny, B., Grzedzielski, M., Janiuk, A., Gu, W-M. et al.: 2016, ApJ 833, Issue 1, article id. $79,6 \mathrm{pp}$.

[281] Yuan, F.: 2016, in Astrophysics of Black Holes, C. Bambi (Ed.), ApSS Library 440, 152-168. 
[282] Zhilkin, A.G., Bisikalo, D.V.: 2009, in Numerical Modeling of Space Plasma Flows: ASTRONUM-2008, Nikolai V. Pogorelov, Edouard Audit, Phillip Colella \& Gary P. Zank (Eds.), ASP Conf. Ser. 406, 118-123.

[283] Zhilkin, A.G., Bisikalo, D.V.: 2010, ARep 54, Issue 12, 1063-1077.

[284] Zhilkin, A.G., Bisikalo, D.V.: 2011, in 5th international conference of numerical modeling of space plasma flows (astronum 2010), Nikolai V. Pogorelov, Edouard Audit \& Gary P. Zank, (Eds.), ASP Conf. Ser. 444, 91-96.

[285] Zhilkin, A.G., Bisikalo, D.V., Mason, P.A.: 2012, ARep 56, Issue 4, 257-274.

[286] Ziółkowski, J.: 2013, Acta Polytechnica Vol 53, Suppl., 665-670.

\section{DISCUSSION}

DMITRY BISIKALO: Debris disks are accretion disks or not? What is your personal view?

FRANCO GIOVANNELLI: The debris disks can be considered, in a broad sense, as accretion disks.

In fact, white dwarfs rich in metals increase their metals from debris disks at a rate up to $10^{11} \mathrm{~g}$ $\mathrm{s}^{-1}$, resulting from the abundances measured in the atmospheres of some white dwarfs. A possible model (Rafikov, 2011) proposes a rapid transport of metals from the debris disk to the white dwarf as a result of the interaction of this with a spatially coexisting metallic gas disk. Under favorable conditions of low viscosity of the metal-gas disk and efficient aerodynamic coupling between the two disks, the system evolves in a runaway fashion, destroying the debris disk in $\sim 10^{5} \mathrm{yr}$, resulting in a high metal accretion rate of $10^{10}-10^{11} \mathrm{~g} \mathrm{~s}^{-1}$, according to the observations.

BOZENA CZERNY's Comment: Pollution problem started at least in medieval times, e.g. the English court changed the castle when it was too dirty (every few months).

BOZENA CZERNY: Accretion onto black hole looks (frequently) similar to accretion onto compact stars, including an apparent similarity of flow with inner ADAF (BH) to intermediate polars. So where the magnetic field forms: in the central object or in the outer disk?

FRANCO GIOVANNELLI: Black holes themselves do not have magnetic poles, and therefore do not generate magnetic fields. This means that the accretion disk corona magnetic fields are somehow generated by the space around a black hole. This process is not well understood at this point.

Black holes expel powerful jets of plasma at near light-speed from the coronae - regions of hot, swirling gas above and below the accretion disc. These coronae and the jets are controlled by powerful magnetic fields - and the stronger the magnetic fields close to the black hole's event horizon, the brighter its jets.

This is because the magnetic fields are thought to act like a synchrotron, accelerating the particles that travel through it. 
Dallilar et al. (2017) studied V404 Cygni's 2015 feeding event across optical, infrared, X-ray and radio wavelengths, and found rapid synchrotron cooling events that allowed them to obtain a precise measurement of the magnetic field.

Their data revealed a much weaker magnetic field than predicted by current models. 